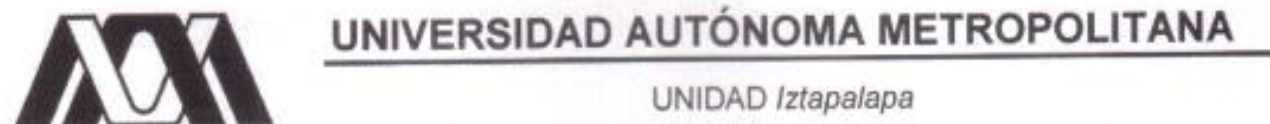 \\ Casa abierta al tiempo \\ DIVISIÓN DE CIENCIAS BÁSICAS E INGENIERIA
}

\section{ESTUDIO DE LA INTERACCIÓN DE MANGIFERINA \\ CON $\beta$-CICLODEXTRINA AL FORMAR COMPLEJOS \\ DE INCLUSIÓN EN MEDIO ACUOSO}

\author{
Tesis que presenta: \\ Q. Lucero Hernández Garcia \\ Para obtener el grado de \\ Maestra en Ciencias (Quimicas)
}

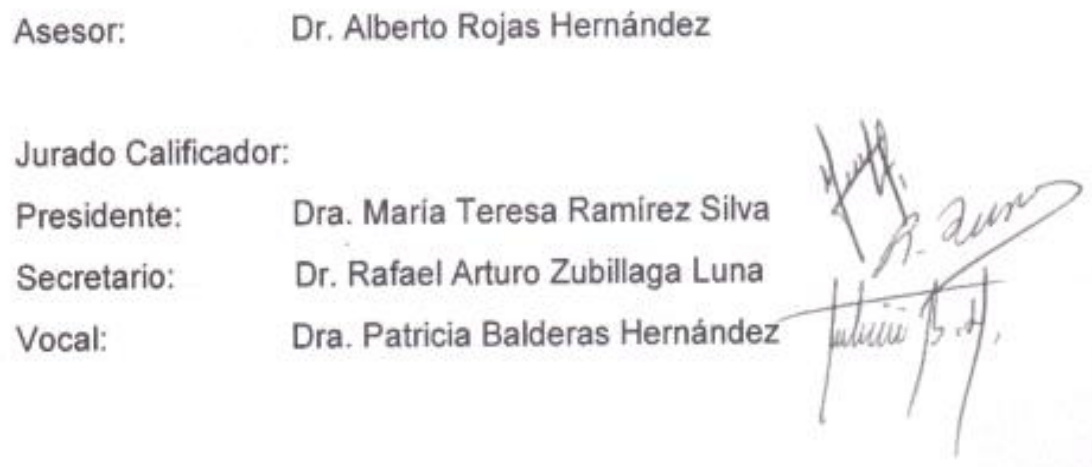

México, D.F. Agosto 2017 


\section{AGRADECIMENTOS}

Al Dr. Alberto Rojas Hernández por su tiempo para dirigir esta tesis, por su paciencia al transmitirme sus conocimientos y su confianza en los proyectos que me tomó en cuenta.

A los sinodales que conformaron el jurado de esta tesis: Dra. María Teresa Ramírez Silva, Dr. Rafael Arturo Zubillaga Luna y Dra. Patricia Balderas Hernández, porque ayudaron a que este trabajo llegara a término de la mejor manera.

A mis padres por su apoyo incondicional que me han brindado, por ser mi ejemplo de vida.

A mis hermanos Mitzi y Alex por su compañía y apoyo moral.

A Jorge por su cariño, apoyo, paciencia y sobre todo por ser parte de mi vida.

A mis amigos y compañeros que me han apoyado con sus criticas sinceras $y$ constructivas.

Al Consejo Nacional de Ciencia y Tecnología (CONACYT) por el financiamiento a través de la beca otorgada para la realización de estudios de posgrado (CVU: 579664 y No. De Becario: 592524 y del proyecto de investigación 237997. Al PRODEP de la SEP, a través de la Red Nacional de Investigación en Química Analítica y Electroquímica (RedNIQAE), también por el financiamiento parcial para el desarrollo de este trabajo. 


\section{CONTENIDO}

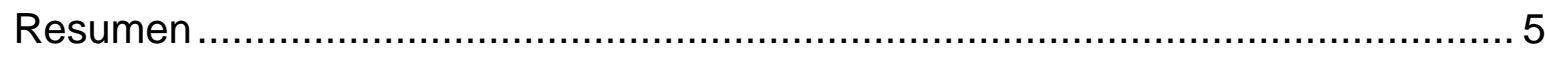

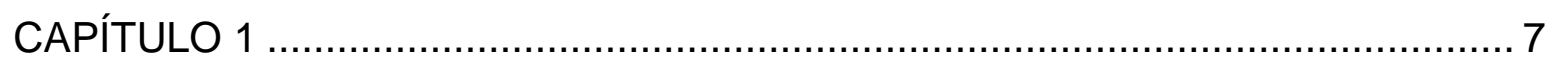

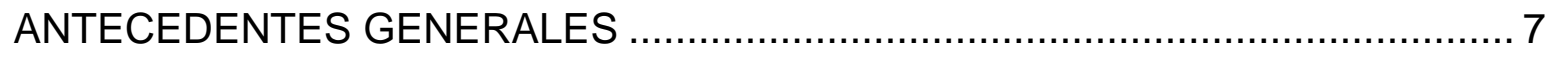

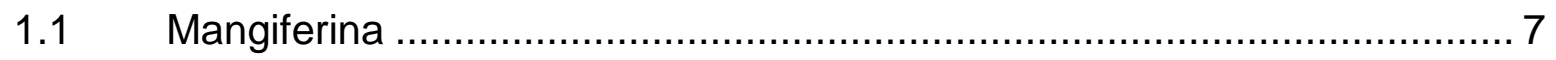

1.2 Tipos de ciclodextrinas y características …............................................... 9

1.3 Complejos de Inclusión ......................................................................... 11

1.3.1 Complejos de inclusión entre MGF con algunos tipos de CDs................ 13

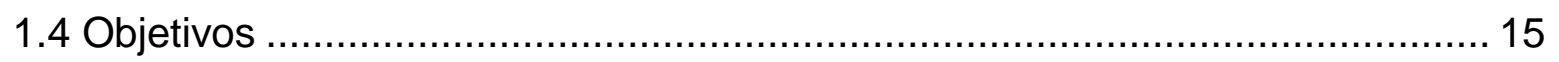

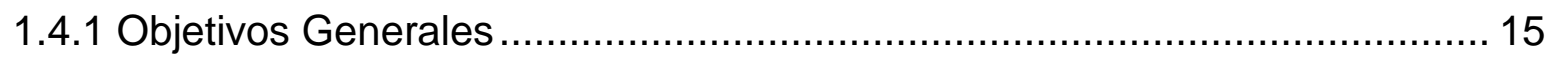

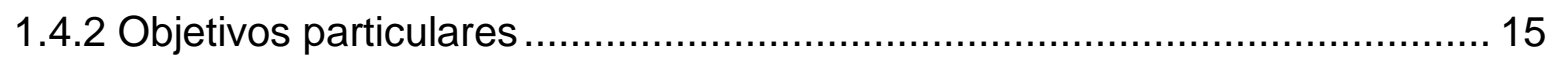

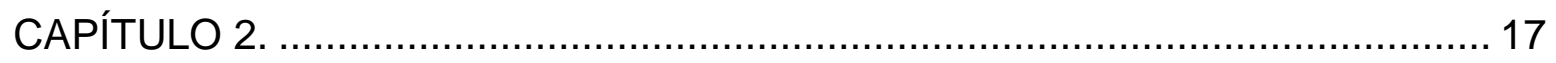

DETERMINACIÓN ESTEQUIOMÉTRICA DE LA FORMACIÓN DEL COMPLEJO DE INCLUSIÓN ENTRE LA $\beta$-CICLODEXTRINA Y LA MANGIFERINA CON SU

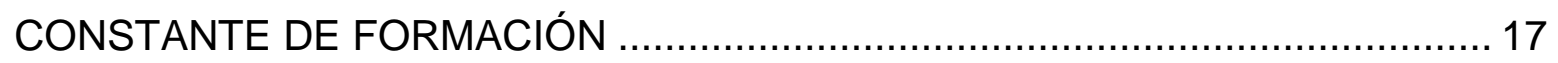

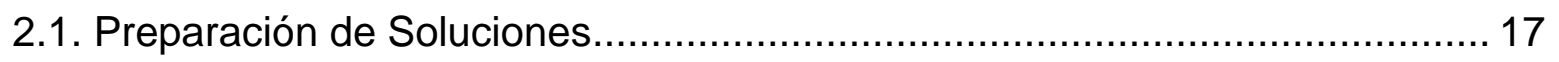

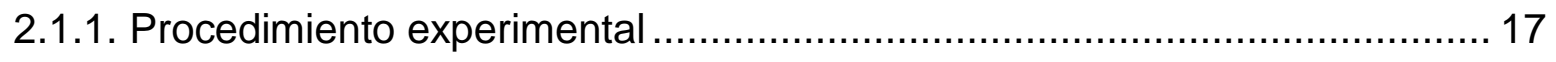

2.2 Resultados y discusión........................................................................ 18

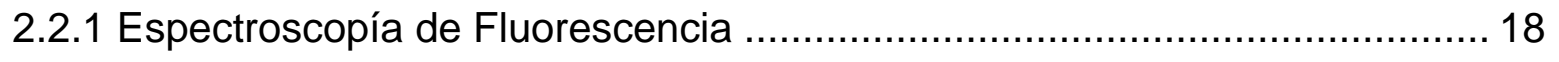

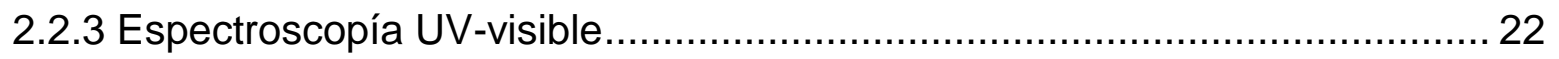

2.3 Determinación de constantes de formación y estequiometría .......................... 24

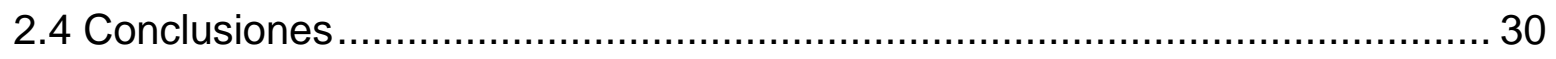

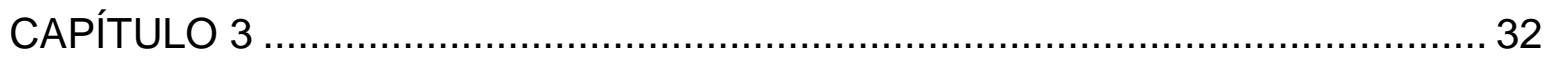

DETERMINACIÓN DE LAS CONSTANTES DE ACIDEZ CONDICIONALES POR ESPECTROFOTOMETRÍA UV-visible PARA MANGIFERINA EN PRESENCIA DE

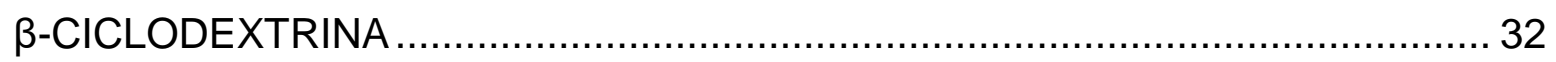




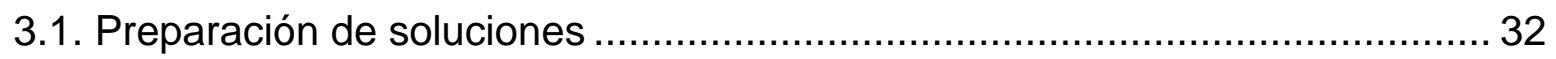

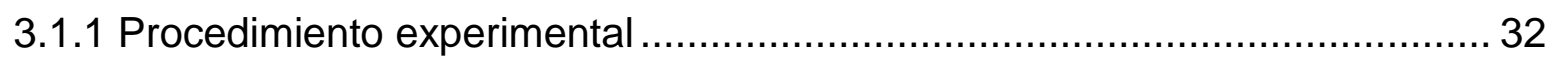

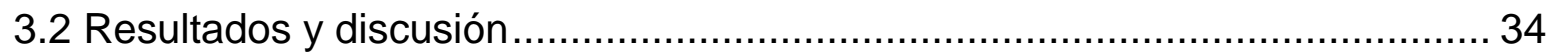

3.2.1 Determinación de espectros de absorción de la mangiferina con $[\beta C D]=1$

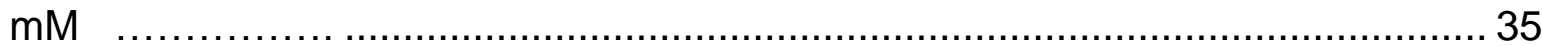

3.2.2 Determinación de constantes de acidez condicionales de la mangiferina con

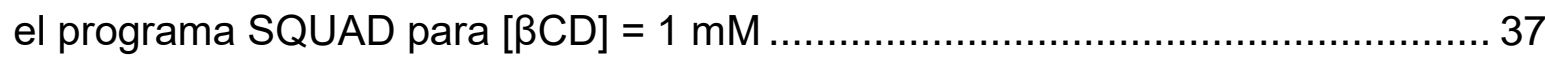

3.2.3 Comparación de los resultados para la mangiferina en ausencia de $\beta C D$ y para

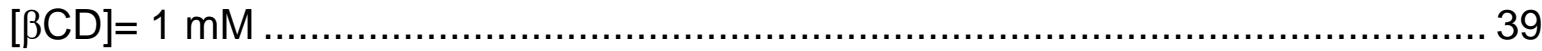

3.2.4 Determinación de espectros de absorción de la mangiferina con $[\beta C D]=10$

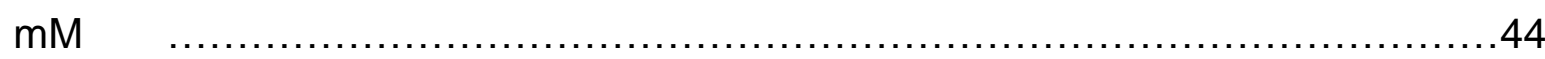

3.2.5 Determinación de constantes de acidez condicionales de la mangiferina con

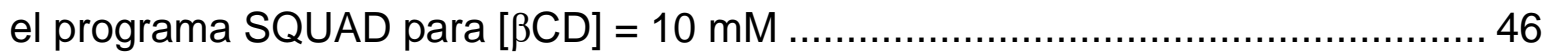

3.2.6 Comparación de los resultados para la mangiferina en ausencia de $\beta C D$ y para

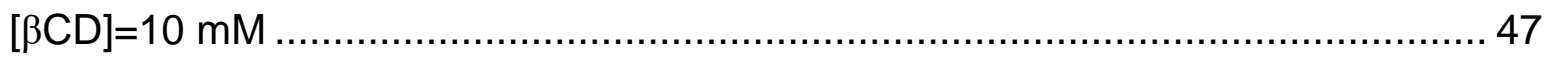

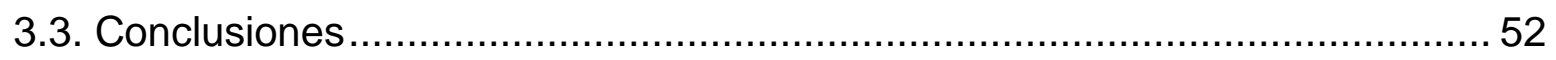

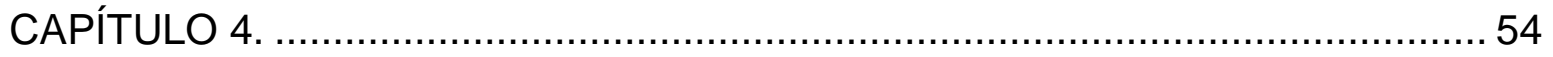

DETERMINACIÓN DE LA CONSTANTE DE INCLUSIÓN DEL COMPLEJO MANGIFERINA/ $\beta$-CICLODEXTRINA POR EL MÉTODO DE HIGUCHI-CONNORS

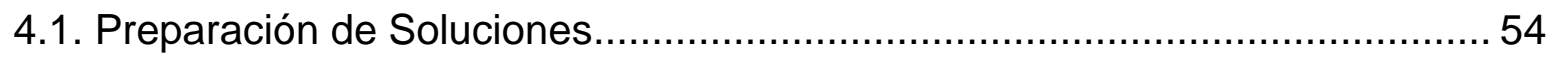

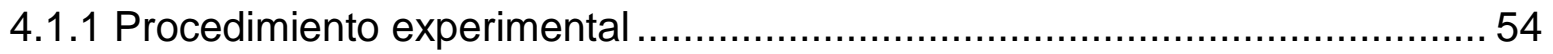

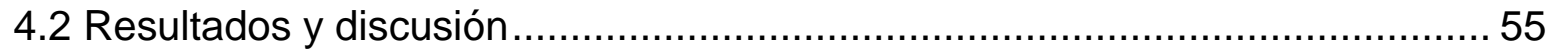

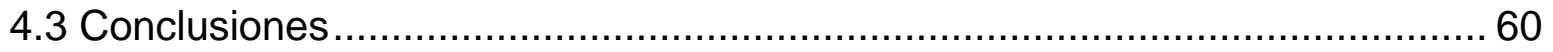

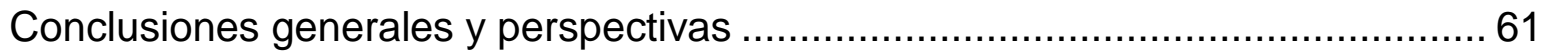

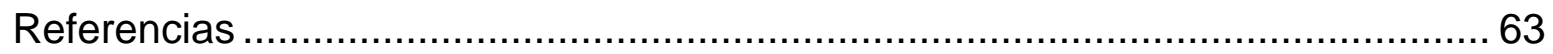




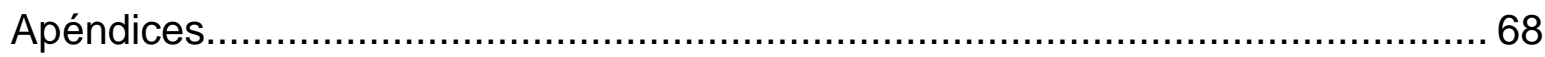

A. Modelo de tres equilibrios para determinar relaciones molares ...............68

B. Análisis de Componentes Principales ….............................................. 70

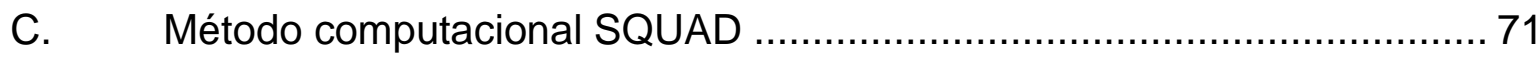

D. Dependencia de las constantes condicionales de acidez con $[\beta C D] \ldots \ldots . .73$

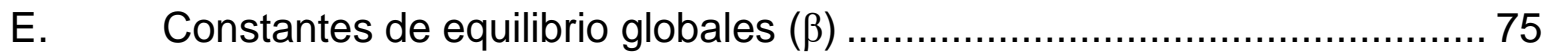

F. Diagramas de distribución de especies para sistemas ácido-base .......... 77

G. Diagrama de solubilidad-fases .................................................... 78

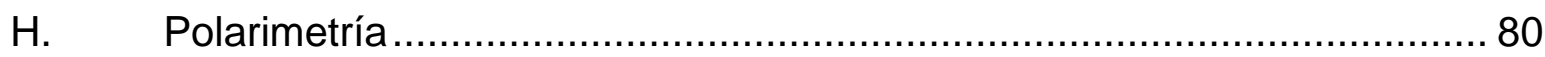




\section{Resumen}

En el presente trabajo se estudió un extracto de la planta del mango, comúnmente conocida como mangiferina, la cual presenta una estructura de tipo xantona glucosilada. De acuerdo con estudios reportados, presenta diferentes acciones farmacológicas ${ }^{5-11}$, pero con algunas limitaciones; entre ellas su baja solubilidad en agua ${ }^{54}$, dicho esto es importante proponer una alternativa que ayude al aumento de esta propiedad con el uso de la formación de complejos de inclusión, específicamente de la $\beta$-CD, ( $\beta$-Ciclodextrina).

Para demostrar la existencia de los posibles complejos de inclusión, se determinaron las cuatro constantes de acidez condicionales de la mangiferina en presencia de la $\beta-C D$, para poder ser comparadas con las constantes de acidez de mangiferina y así ver el efecto sobre estas últimas, dicho experimento se llevó a cabo en medio acuoso por Espectroscopía UV-visible a $25.0 \pm 0.5^{\circ} \mathrm{C}$.

Después, por las técnicas de Espectroscopía UV-visible y Fluorescencia, se determinó la estequiometría del complejo formado usando un método robusto con tres posibilidades diferentes de unión Huésped:Anfitrión con las relaciones molares (1:1, $1: 2$ y $2: 1)$, donde para el tratamiento de los datos se usó el programa computacional SQUAD; obteniendo en este trabajo la relación molar 1:1 con su respectiva constante de inclusión.

Una de las propiedades que presenta la mangiferina es su baja solubilidad en agua, por lo que; para ser usada en procesos biológicos es necesario utilizar concentraciones más altas de su valor de solubilidad, debido a esto fue importante determinar el aumento en la solubilidad de la mangiferina al formar complejos de inclusión con $\beta-C D$ ( $\beta$-ciclodextrina), sabiendo que esta molécula puede cambiar la solubilidad de otras (Huésped). Existe un método para determinar la constante de solubilidad, propuesto por Higuchi y Connors $^{42}$, este estudio se basa en la construcción de un diagrama de fases que se construye a partir de la solubilidad de mangiferina en función de la concentración de $\beta-C D$, para éste trabajo con un experimento clasificado de Tipo $A$, según la definición del método, que además fue seguido por espectroscopía UV-visible y Polarimetría en medio acuoso. 


\section{CAPÍTULO 1}

\section{ANTECEDENTES GENERALES}




\section{CAPÍTULO 1}

\section{ANTECEDENTES GENERALES}

\section{$1.1 \quad$ Mangiferina}

La mangiferina ( $\mathrm{H}_{4} \mathrm{MGF}$ ), es un polifenol de origen natural que representa la estructura de 2- $\beta$-D-glucopiranosil-1, 3, 6, 7-tetrahidroxixanten-9-ona, una xantona C-glucosilada, Figura 1.1.1; está presente en mayor concentración en especies de Mangifera Indica L. (nombre común: mango), ésta pertenece a la familia de Anacardiaceae y es la fuente de muchos compuestos naturales entre ellos xantonas y flavonoides. Aunque reportes indican que también puede estar distribuida en plantas como son Cyclopia y Salacia ${ }^{1}$.<smiles>O=c1c2cc(O)c(O)cc2oc2cc(O)c(C(O)[C@H](O)[C@H](O)CO)c(O)c12</smiles>

Figura 1.1.1 Estructura de la mangiferina, 2- $\beta$-D-glucopiranosil-1, 3, 6, 7-tetrahidroxixanten-9-ona 5 .

La estructura de $\mathrm{H}_{4}$ MGF satisface las reglas de Lipinski², que analiza las propiedades fisicoquímicas, para poder ser propuestos como fármacos y facilite la absorción en el cuerpo ${ }^{3}$. Dichas propiedades deben cumplir un peso molecular (MW) menor a $500 \mathrm{~g} / \mathrm{mol}$, un coeficiente de partición octanol / agua con $\log P \leq 5$, con enlaces- $\mathrm{H}$ donantes $\leq 5 \mathrm{y} \leq 10$ enlaces $-\mathrm{H}$ aceptores, para la mangiferina indican que satisface estas propiedades con un MW de $422.33 \mathrm{~g} / \mathrm{mol}$, un $\log P=2.73$, y 4 funciones de donante para enlaces de hidrógeno, 2 funciones aceptoras para enlaces de hidrógeno ${ }^{4}$, lo que indica que tiene potencial para ser modificado por la química medicinal para alterar sus funciones y propiedades ${ }^{5}$. 
Debido a lo anterior, existen algunos estudios que reportan que la mangiferina posee propiedades analgésicas, antidiabéticas ${ }^{6}$, antiescleróticas, antimicrobianas, antivirales, cardiohepatoprotectoras, antiinflamatorias, antialérgicas y antioxidantes ${ }^{7}$. Se vende para el tratamiento del trastorno metabólico en Cuba bajo el nombre comercial de Vimang ${ }^{\circledR} 8$-9.

Los mecanismos propuestos para entender las bioactividades de la mangiferina se enfocan en su capacidad para proveer protección celular ${ }^{10}$, debido a que presenta un grupo catecol, y a que contiene cuatro grupos hidroxilo con la función de ser un eficiente antioxidante, captador de radicales libres e inmunomodulatorias ${ }^{11}$.

Los trabajos reportados por Gómez-Zaleta ${ }^{12}$ y Mendoza-Sarmiento ${ }^{13}$, junto con sus respectivos colaboradores, determinaron las constantes de acidez en solución acuosa de la mangiferina, Tabla 1.1, y proponen su orden de desprotonación con un estudio de resonancia magnética nuclear ${ }^{13}$ Figura 1.1.2.

Tabla 1.1 Valores de pKa para la Mangiferina reportados en la literatura obtenidos por UV-visible.

\begin{tabular}{ccc}
\hline Equilibrio & \multicolumn{2}{c}{ Valor reportado } \\
& Gómez-Zaleta $^{12}$ & Mendoza-Sarmiento $^{13}$ \\
\hline$H_{4}(M G F)=H_{3}(M G F)^{-}+H^{+}$ & $\mathrm{pK}_{\mathrm{a} 1=6.52 \pm 0.06}$ & $\mathrm{pK}_{\mathrm{a} 1}=6.345 \pm 0.036$ \\
$H_{3}(M G F)=H_{2}(M G F)^{2-}+H^{+}$ & $\mathrm{pK}_{\mathrm{a} 2=7.97 \pm 0.06}$ & $\mathrm{pK}_{\mathrm{a} 2}=7.953 \pm 0.045$ \\
$H_{2}(M G F)=H(M G F)^{3-}+H^{+}$ & $\mathrm{pK}_{\mathrm{a} 3=9.44 \pm 0.04}$ & $\mathrm{pK}_{\mathrm{a} 3=9.571 \pm 0.049}$ \\
$H(M G F)=(M G F)^{4-}+H^{+}$ & $\mathrm{pK}_{\mathrm{a} 4=12.10 \pm 0.01}$ & $\mathrm{No}$ se determino \\
& $\sigma_{\mathrm{reg}=4.92 \times 10^{-3}}$ & $\sigma_{\mathrm{reg}=7.072 \times 10^{-3}}$ \\
& $\mathrm{U}=2.20 \times 10^{-2}$ & $\mathrm{U}=3.471 \times 10^{-2}$
\end{tabular}

* Las constantes fueron determinadas por la técnica de Espectroscopía UV-vis, en medio acuoso y atmósfera inerte de $\mathrm{N}_{2}$. 


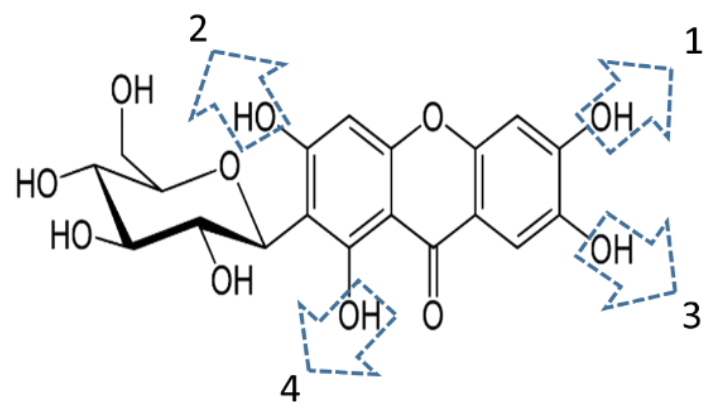

Figura 1.1.2. Orden de desprotonación de los sitios ácidos presentes en la mangiferina, determinados por resonancia magnética nuclear, por Mendoza-Sarmiento ${ }^{13}$.

Una característica principal de la mangiferina es que presenta poca solubilidad en agua y baja biodisponibilidad, lo que limita algunas de las aplicaciones farmacéuticas anteriormente mencionadas, entre los estudios informan de la mala absorción oral de $\mathrm{H}_{4} \mathrm{MGF}^{14-15}$. Desde diferentes enfoques se han empleado experimentos que mejoran la solubilidad de la mangiferina, entre ellos existen sistemas microencapsulados ${ }^{16}$ y nanocápsulas ${ }^{17}$, entre otros.

\subsection{Tipos de ciclodextrinas y características}

Las ciclodextrinas (CDs) son oligosacáridos cíclicos (producidos en organismos vivos a partir de la degradación del almidón con la enzima ciclodextrina glucanotransferasa (CGtasa), que presentan una estructura formada por grupos glucosídicos unidos en sus posiciones $(\alpha-1,4)^{18-19}$. Los tipos de CDs más comunes son $\alpha C D, \beta C D$ y $\gamma C D$, que se componen de 6,7 y 8 moléculas de glucosa, respectivamente ${ }^{18}$.

La cavidad está revestida por los átomos de hidrógeno y los puentes glucosídicos de oxígeno que tienen pares de electrones no enlazantes y están dirigidos hacia el interior de la cavidad produciendo una alta densidad de electrones ${ }^{20}$. Además las estructuras tienen los grupos hidroxilos secundarios (C-2 y C-3) situados en el borde más ancho del anillo y los grupos hidroxilo primarios (C-6) en el borde más angosto, 
los hidrógenos de los C-3 y C-5 son apolares y los oxígenos de tipo éter están en el interior de las moléculas en forma de cono. Esto resulta en una molécula con un exterior hidrófilo, que puede disolverse en el agua, y una cavidad apolar que proporciona una matriz hidrófoba Figura 1.2.1 (b) ${ }^{20}$.

La hidrofobicidad de la cavidad interna permite que las CDs puedan asociarse con moléculas orgánicas no polares parcial o completamente, para formar complejos de inclusión. Existen diferentes tipos de CDs, Figura 1.2.1. (a), unas dependen del número de glucosas unidas y otras de grupos alifáticos sustituidos; entre ellas está la $\beta C D$ que contiene 7 unidades de glucosa en su formación cíclica, es en particular la menos soluble en solución acuosa con un valor de $1.85 \mathrm{~g} / \mathrm{mL}$, comparada con la aCD (6 unidades de glucosa) y $\gamma \mathrm{CD}$ (8 unidades de glucosa) con un valor de solubilidad de $14.5 \mathrm{~g} / \mathrm{mL}$ y $23.2 \mathrm{~g} / \mathrm{mL}$, respectivamente.

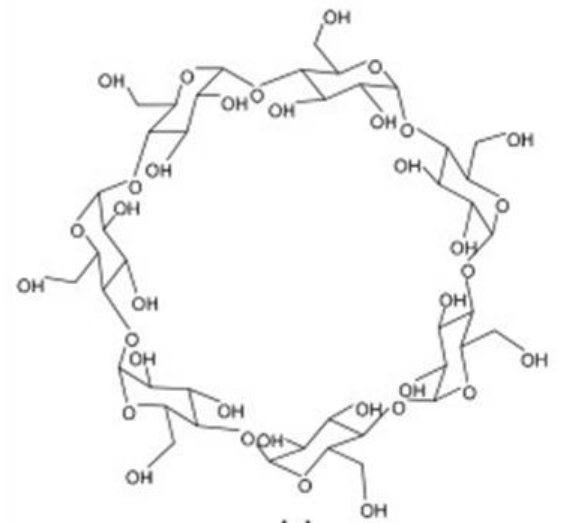

[a]

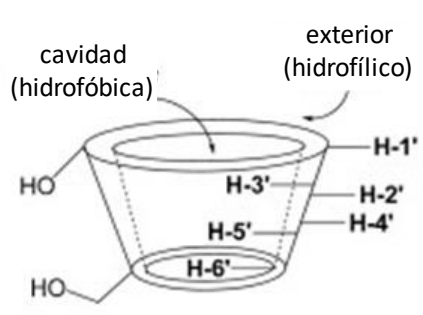

[b]

Figura 1.2.1. Estructura química de ciclodextrina: (a) Representación de la estructura de $\beta$-CD (7 unidades de glucosa). (b) Representación sistemática de la cavidad en forma de anillo o cono truncado y las regiones hidrofóbicas e hidrofílicas ${ }^{19}$.

En la Tabla 1.2 se describen algunas propiedades de las CDs y sus diferencias. Los principales tipos de ciclodextrinas ya se mencionó que presentan diferentes propiedades químicas pero la $\beta-C D$ es la más accesible económicamente en comparación con las otras y generalmente es la más utilizada. 
Tabla 1.2. Características químicas de $\alpha, \beta$ y $\gamma-$ CDs.

\begin{tabular}{lccc}
\hline \multicolumn{1}{c}{ Propiedades $^{19}$} & $\boldsymbol{\alpha}-\boldsymbol{C D}$ & $\boldsymbol{\beta}-\boldsymbol{C D}$ & $\boldsymbol{\gamma}-\boldsymbol{C D}$ \\
\hline Número de unidades de glucosa & 6 & 7 & 8 \\
Peso molecular / $\mathrm{g} \mathrm{mol} \mathrm{I}^{-1}$ & 972 & 1135 & 1297 \\
Solubilidad en agua $/ \mathrm{g} \mathrm{dL}^{-1} 25^{\circ} \mathrm{C}$ & 14.5 & 1.85 & 23.2 \\
{$[\alpha]_{D} 25^{\circ} \mathrm{C}$} & $150 \pm 0.5$ & $162.5 \pm 0.5$ & $177.4 \pm 0.5$ \\
Diámetro de la cavidad /Á & $4.7-5.3$ & $6.0-6.5$ & $7.5-8.3$ \\
Altura del cono /Á & $7.9 \pm 0.1$ & $7.9 \pm 0.1$ & $7.9 \pm 0.1$ \\
pKa (por potenciometría) $25^{\circ} \mathrm{C}$ & 12.3 & $13.2^{21}, 12.2^{22}$ & 12.1 \\
\hline
\end{tabular}

\subsection{Complejos de Inclusión}

La química supramolecular es una disciplina de la química que implica todas las interacciones intermoleculares en las que no se establecen enlaces covalentes entre las especies que interactúan a través de muchas interacciones débiles entre moléculas, iones o radicales ${ }^{21,22}$

Las CDs forman un tipo particular de compuestos denominados "complejos de inclusión", los cuales están formados por una molécula llamada "huésped", que típicamente se dice que se aloja en la cavidad de las CDs, que entonces se consideran como "anfitrión".

Para que pueda existir la formación de los complejos de inclusión deben existir interacciones como son las fuerzas de Van der Waals, interacciones hidrofóbicas, puentes de hidrógeno y efectos estéricos, por mencionar algunas, las cuales provocan cambios importantes en las propiedades de las moléculas orgánicas introducidas en las CDs, tales como aumentar su solubilidad, reactividad química, conformación estructural, entre otras ${ }^{23}$. Por otra parte, las CDs en solución disminuyen la volatilidad del solvente. Por estas razones, las CDs tienen una gran aplicación en la industria química, por ejemplo, en la industria farmacéutica se utiliza como un sistema de liberación y solubilización de fármacos, en la industria 
alimentaría como enmascarante de olores y sabores, en el área biológica para mimetizar sitios activos de enzimas e imitar de algún modo los mecanismos de reconocimiento molecular que forman parte importante de los procesos bioquímicos en la catálisis enzimática que se lleva a cabo en los organismos vivos ${ }^{24}$.

También se han utilizado las CDs en la fabricación de biosensores, entre otras aplicaciones ${ }^{25}$.

Al interactuar la molécula huésped $(G)$ con la molécula anfitrión (CD), reacciona para formar los complejos de inclusión. La estequiometría más común de este tipo de compuestos es la relación 1G:1CD ${ }^{26}$, sin embargo, existen evidencias de la asociación con 2G:1CD, 1G:2CD, 2G:2CD 27,28,29.

Algunos complejos de inclusión pueden ser aislados como sustancias cristalinas estables. En disolución, los complejos también se pueden estabilizar debido a las mùltiples interacciones débiles anteriormente mencionadas ${ }^{30,31}$, estableciendo un equilibrio químico entre las especies asociadas $G_{m}(C D)_{n}$ y las disociadas, $G$ y $C D$, mediante la constante de estabilidad de cada complejo formado (K), ecuación 1.1:

$$
\begin{gathered}
m G+n C D \rightarrow G_{m}(C D)_{n} \\
K_{m: n}=\frac{\left[G_{m}(C D)_{n}\right]}{[G]^{m}[C D]^{n}}
\end{gathered}
$$

Las interacciones entre un huésped poco soluble y una CD en solución acuosa provocan algunos de los siguientes efectos:

$\checkmark$ La concentración o solubilidad del huésped en fase acuosa se incrementa significativamente, por lo que es posible que a partir de huéspedes ionizados o compuestos estabilizados por enlaces de hidrógeno podría favorecerse el incremento de la solubilidad de los huéspedes por medio de las $\mathrm{CDs}^{32}$. 
$\checkmark$ Las propiedades espectrales del huésped se modifican. Por ejemplo, los desplazamientos químicos de los átomos se modifican en los espectros de RMN.

$\checkmark$ También cuando los huéspedes que absorben luz se insertan en la cavidad quiral de las CDs, la respuesta de la rotación óptica (de las CDs) muestra un fuerte efecto de Cotton inducidos en los espectros de dicroísmo circular.

$\checkmark$ A veces, la longitud de onda del máximo, de los espectros de UV-visible, se desplaza en unidades o hasta en decenas de nanómetros.

$\checkmark$ La Fluorescencia de los huéspedes también tiende a incrementarse, debido a que la molécula fluorescente se transfiere desde el medio acuoso a un entorno apolar que lo hace más rígido ${ }^{21}$.

$\checkmark$ El huésped, anteriormente hidrofóbico, se podría volver hidrófilo después de la complejación con las CDs; por lo que también se modifica su movilidad cromatográfica o la separación química en medios polares y apolares.

\subsubsection{Complejos de inclusión entre MGF con algunos tipos de CDs}

Para la formación de complejos de inclusión las moléculas huésped deben tener ciertas características físicas y químicas, entre ellas está ser una molécula con una parte hidrofóbica, así que la variedad de moléculas huéspedes es muy amplia. La inclusión de este tipo de moléculas puede ser total o parcial, dependiendo de las fuerzas fisicoquímicas involucradas.

Estudios con diferentes moléculas orgánicas desde la perspectiva farmacológica, dejan la puerta abierta para utilizar la mangiferina, misma para la que existen algunos trabajos que estudian la formación de complejos de inclusión con ciertos tipos de CDs. A continuación se presentan los más relevantes:

$\checkmark$ Yang y colaboradores, utilizaron derivados de $\beta$-CD, 2-hidroxipropil- $\beta$ ciclodextrina (HP- $\beta-C D)$, sulfobutiléter- $\beta$-ciclodextrina (SBE- $\beta-C D)$ y mono 
(6-etilendiamino-6-dioxi- $\beta$-ciclodextrina $(E N-\beta-C D)$, con la finalidad de determinar la solubilidad y la estabilidad por métodos de espectroscopía NMR de $\mathrm{H}$, y de difracción de rayos $\mathrm{X}(\mathrm{XRD})$. Además estimando una estequiometría por cálculos matemáticos y aproximaciones gráficas por el método variaciones continuas (o método de Job) usando la técnica de espectroscopía de Fluorescencia ${ }^{33}$.

$\checkmark$ Huang y colaboradores, usaron un sistema de $\beta$-CD sustituida con grupos dietilo (DE- $\beta-C D$ ) y mangiferina (MGF) por la técnica de Fluorescencia, proponiendo una estequiometría 1MGF:2DE- $\beta-C D$, y el uso de un amortiguador a $\mathrm{pH}=8$ de $\mathrm{H}_{3} \mathrm{BO}_{3}-\mathrm{KCl}-\mathrm{NaOH}$. Con una aproximación gráfica del recíproco de la intensidad de Fluorescencia en función del inverso de la concentración de DE- $\beta-C D^{34}$. 


\subsection{Objetivos}

\subsubsection{Objetivos Generales}

Determinar las constantes de formación para complejos de inclusión formados entre la $\beta C D$ y la MGF', en solución acuosa, por técnicas espectrofotométricas.

\subsubsection{Objetivos particulares}

* Determinar la estequiometría de MGF': $\beta C D$, por Espectrofotometría UVvisible y por Fluorescencia, a un valor de $\mathrm{pH}$ impuesto (8.6).

* Determinar las constantes de acidez condicionales del complejo de inclusión con relación molar 1:1, por Espectrofotometría UV-visible, con el uso del programa computacional SQUAD. Utilizando un método robusto de tres equilibrios.

* Conocer la constante de inclusión para la formación de complejos entre mangiferina y $\beta C D$, usando el método reportado por Higuchi y Connors, por medio del estudio del diagrama de solubilidad-fases. 


\section{CAPÍTULO 2}

\section{DETERMINACIÓN ESTEQUIOMÉTRICA DE LA FORMACIÓN DEL COMPLEJO DE INCLUSIÓN ENTRE LA $\beta$-CICLODEXTRINA Y LA MANGIFERINA CON SU CONSTANTE DE FORMACIÓN}




\section{CAPÍTULO 2.}

\section{DETERMINACIÓN ESTEQUIOMÉTRICA DE LA FORMACIÓN DEL COMPLEJO DE INCLUSIÓN ENTRE LA $\beta$-CICLODEXTRINA Y LA MANGIFERINA CON SU CONSTANTE DE FORMACIÓN}

La determinación de la estequiometría y las constantes de formación de complejos de inclusión entre mangiferina y $\beta$-ciclodextrina fue seguida por dos técnicas en soluciones acuosas: la espectrofotometría UV-visible y la Fluorescencia. Con la finalidad de tener evidencia de la formación de complejos de inclusión, se trabajó con soluciones en donde la concentración de mangiferina se mantuvo fija, variando la concentración de $\beta C D$.

\subsection{Preparación de Soluciones}

Se llevó a cabo la preparación de una solución de H4MGF (Sigma-Aldrich, un extracto), de $0.038 \mathrm{mM}$ en $50 \mathrm{~mL}$ de agua desionizada; ajustando el valor de $\mathrm{pH}$ en aproximadamente $8.600 \pm 0.001$ con una solución de $\mathrm{NaOH}(1 \mathrm{~N}$, Baker) a temperatura ambiente.

Posteriormente se preparó una solución de $\beta C D$ (Sigma-Aldrich, $95 \%$ ), de concentración $0.01 \mathrm{M}$ en $25 \mathrm{~mL}$, con agua desionizada, del mismo modo ajustando el valor de $\mathrm{pH}$ en 8.6 .

\subsubsection{Procedimiento experimental}

A partir de las soluciones de $\mathrm{H}_{4} \mathrm{MGF}$ y $\beta C D$ anteriormente descritas, se prepararon diferentes soluciones donde se mantuvo fija la concentración de mangiferina en 0.01 $\mathrm{mM}$ y en cada una se fue variando la concentración de $\beta C D$ en un intervalo de $6.00 \times 10^{-4} \mathrm{mM}$ hasta $9.28 \mathrm{mM}$; cada mezcla se llevó a $10 \mathrm{~mL}$ con agua desionizada; inmediatamente se colocó en un baño de ultrasonido durante 45 min a temperatura ambiente. Una vez transcurrido ese tiempo se verificó el valor de pH en 8.6 y en los 
casos donde hubo cambios de aumento o disminución del mismo se procedió al ajuste con $\mathrm{HCl}$ y $\mathrm{NaOH}$ concentrados, respectivamente.

Posteriormente a cada solución se le midió su espectro de absorción UV-visible, en un intervalo de 240 a $450 \mathrm{~nm}$ en un equipo Perkin Elmer Lambda 950 y por Fluorescencia con un equipo Perkin Elmer LS 55, midiendo en esta última técnica los espectros de excitación y emisión a ciertas condiciones de $\lambda$ emisión y $\lambda_{\text {excitación, }}$ respectivamente.

El pH se midió con un equipo $\mathrm{HACH}$ sensION+pH 31, equipado con un electrodo combinado de vidrio con referencia externa de $\mathrm{Ag} / \mathrm{AgCl}$, que permite medir valores de $\mathrm{pH} 0$ a 14.

\subsection{Resultados y discusión}

Las constantes de inclusión pueden estudiarse a diferentes valores de $\mathrm{pH}$, aunque en este trabajo se presentan solamente los resultados obtenidos cuando el $\mathrm{pH}$ de las soluciones es 8.6. En estas condiciones, la especie francamente predominante (aproximadamente en un $70 \%$ ) es la diprotonada: $\mathrm{H}_{2} \mathrm{MGF}^{2-12,13}$. Es por eso que en este capítulo 2 se define la especie generalizada, MGF' tal que:

$$
\left[\mathrm{MGF}^{\prime}\right]=\left[\mathrm{H}_{4} \mathrm{MGF}\right]+\left[\mathrm{H}_{3} \mathrm{MGF}^{-}\right]+\left[\mathrm{H}_{2} \mathrm{MGF}^{2-}\right]+\left[\mathrm{HMGF}^{3-}\right]+\left[\mathrm{MGF}^{4-}\right] \approx\left[\mathrm{H}_{2} \mathrm{MGF}^{2-}\right] .
$$

\subsubsection{Espectroscopía de Fluorescencia}

La Espectroscopía de Fluorescencia es una herramienta poderosa para cuantificar los sistemas moleculares del tipo huésped-anfitrión con alta sensibilidad y alta selectividad, permitiendo con frecuencia medir concentraciones mucho más bajas que en el análisis espectrofotométrico ${ }^{35-36}$. En la espectroscopía de Fluorescencia se pueden obtener dos tipos de espectros, clasificados como de excitación (que 
tiene el mismo perfil de un espectro de absorción) y de emisión, para los cuales se buscaron las condiciones apropiadas de trabajo. Para los espectros de excitación se colocó el monocromador de emisión a una longitud de onda, $\lambda_{\mathrm{em}}$, de $530 \mathrm{~nm}$, abriendo las rendijas (slits) de excitación y emisión a $5 \mathrm{~nm}$, y variando el monocromador de excitación en el intervalo de 300 a $460 \mathrm{~nm}$. En el caso de los espectros de emisión se hizo incidir luz de $\lambda_{\mathrm{ex}}=378 \mathrm{~nm}$ sobre la muestra, abriendo también ambas rendijas a $5 \mathrm{~nm}$ y variando el monocromador de emisión de 440 a $680 \mathrm{~nm}$. Con estas condiciones se obtuvieron los espectros de Fluorescencia descritos al inicio del capítulo que se muestran en la Figura 2.2.1.

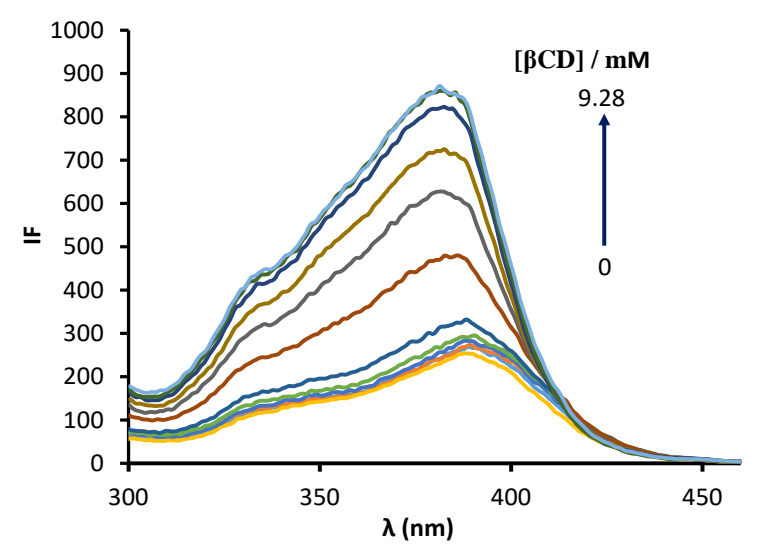

(a)

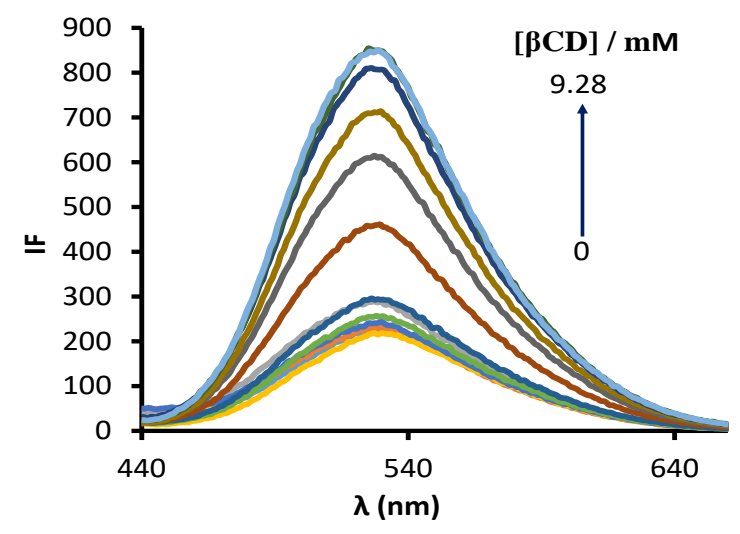

(b)

Figura 2.2.1. Aumento en los espectros de Fluorescencia al aumentar la concentración de $\beta$-CD' desde $0 \mathrm{mM}$ hasta $9.28 \mathrm{mM}$ cuando $\left[\mathrm{MGF}^{\prime}\right]=1 \times 10^{-2} \mathrm{mM}$, a $\mathrm{pH}=8.6$. Las curvas en (a) presentan los espectros de excitación con $\lambda_{\text {em }}=530 \mathrm{~nm} / 5 \mathrm{~nm}$ de $300-460 \mathrm{~nm}$. Las curvas en (b) representan los espectros de emisión a $\lambda_{e x}=378 \mathrm{~nm} / 5 \mathrm{~nm}$ de $440-680 \mathrm{~nm}$. 
En la figura 2.2.1. se puede observar un aumento en la intensidad de Fluorescencia conforme se fueron realizando adiciones de $\beta C D$. Esta tendencia fue igual tanto en los espectros de excitación como en los espectros de emisión. Con esta información se procede a realizar la construcción de los gráficos de intensidad de Fluorescencia en función de la concentración total de $\beta C D$, tomando las longitudes de onda donde se observa el máximo en la intensidad, para los espectros de excitación (en 384 nm) y en los espectros de emisión (a $528 \mathrm{~nm}$ ).

Comúnmente, en el método de relaciones molares se construye la curva de intensidad de Fluorescencia (o la respuesta) en función del cociente de concentraciones de $[\beta C D] /[M G F ']$, como se muestra en la Figura 2.2.2a. La curva observada en dicha figura no tiene la forma esperada en donde se pueden encontrar segmentos lineales en donde la intersección de dichos segmentos señala la relación estequiométrica de complejos de inclusión. Esto puede deberse a que hay varios procesos involucrados correspondientes a reacciones muy poco cuantitativas, lo que impide usar la curva mostrada en la figura 2.2.2.a para determinar la estequiometría de los complejos formados. En el apéndice $A$ de este trabajo se muestra que en este caso la curva 2.2.2.b puede dar mayor información del sistema.

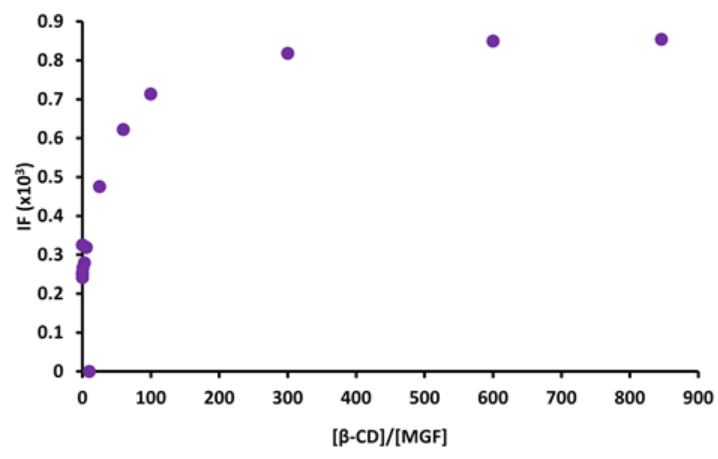

(a)

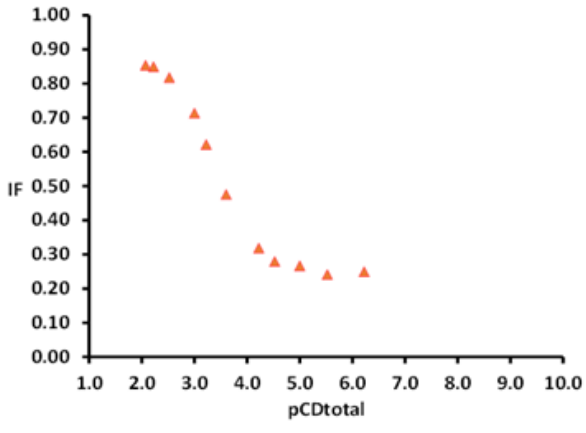

(b)

Figura 2.2.2. Curva de relaciones molares de intensidad de Fluorescencia como función del cociente de concentraciones de $[\beta C D] /[M F G ']$ para $\lambda_{e x}=378 \mathrm{~nm}$ y $\lambda_{e m}=528 \mathrm{~nm}$ a $\mathrm{pH}=8.6$. a) Representación típica de la curva de realciones molares. b) Curva de IF como función del $-\log [C D]_{t o t a l}$. 
Dado que no es fácil estimar las características principales de los complejos de inclusión formados a partir de curvas como las que se muestran en la figura 2.2.2. se decidió hacer un estudio similar al que se hace de los espectros de absorción, con los espectros de Fluorescencia.

Así, con ayuda del algoritmo de análisis de componentes principales (PCA por sus siglas en inglés), se puede estimar el número de especies que presentan Fluorescencia en el sistema (ver Apéndice B). Para aplicar el PCA se utilizó el programa Minitab16 ${ }^{37}$.

En la Tabla 2.1 y en la Figura 2.2.3 se muestran los resultados relevantes del PCA para estimar el número de especies en el sistema que presentan diferente capacidad de Fluorescencia.

Tabla 2.1. Porcentaje de varianza acumulada para los primeros componentes principales, $\mathrm{PC}$, obtenidos con el algoritmo de $\mathrm{PCA}^{38}$.

\begin{tabular}{r|r|l|l|l}
\hline \multicolumn{2}{|c|}{} & \multicolumn{3}{|c}{ PC } \\
\hline Espectros de & & \multicolumn{1}{c}{1} & \multicolumn{1}{c}{3} \\
\hline EMISIÓN & Porcentaje de & 99.6 & 100.0 & 100.0 \\
EXCITACIÓN & varianza acumulada & 78 & 100 & 100
\end{tabular}

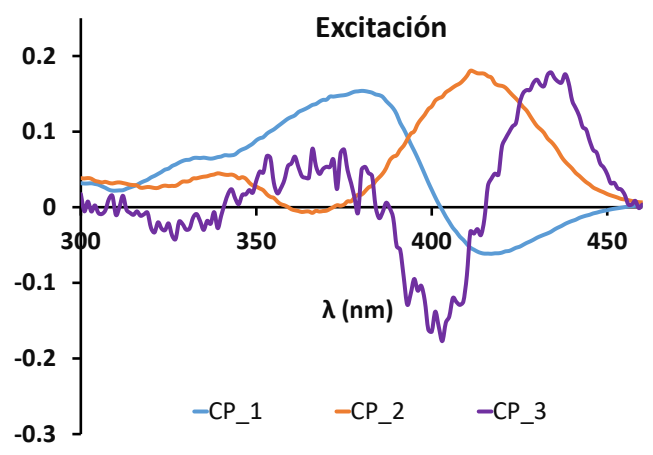

(a)

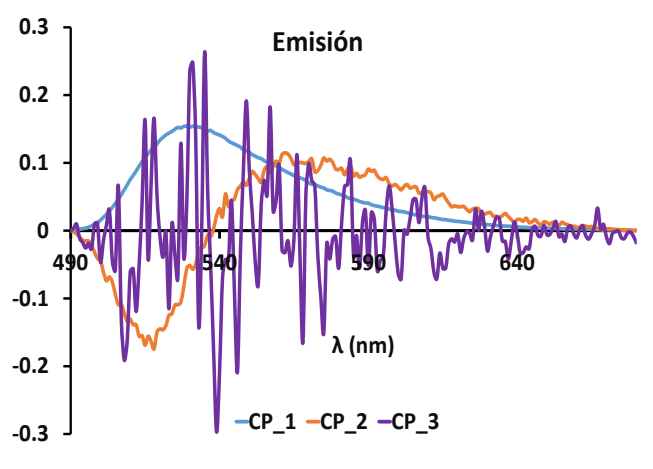

(b)

Figura 2.2.3. Gráfica de "loadings" en función de la longitud de onda obtenidos por PCA, para espectros medidos por Fluorescencia. En (a) se muestran los loadings para espectros de excitación y en (b) son presentan los loadings para espectros de emisión, que se encuentran en la Figura 2.2.1. 
En la Tabla 2.1 se muestra que basta usar dos componentes para reconstruir el 100 $\%$ de la información relevante. Además en la Figura 2.2.3 se observa mucho ruido a partir del tercer componente. Estos resultados se identifican con la presencia de dos especies en el sistema con diferente capacidad de Fluorescencia.

\subsubsection{Espectroscopía UV-visible}

Para las soluciones cuya preparación se describe en este capítulo se obtuvieron los espectros de absorción que se presentan en la Figura 2.2.4. A pesar de que los espectros de absorción no presentan cambios significativos en la absorbancia de la solución de MGF' $\sin \beta-C D$ a la que contiene $\beta-C D$ en exceso, sólo se observa un ligero desplazamiento a longitudes de onda más cortas (hipsocrómico).

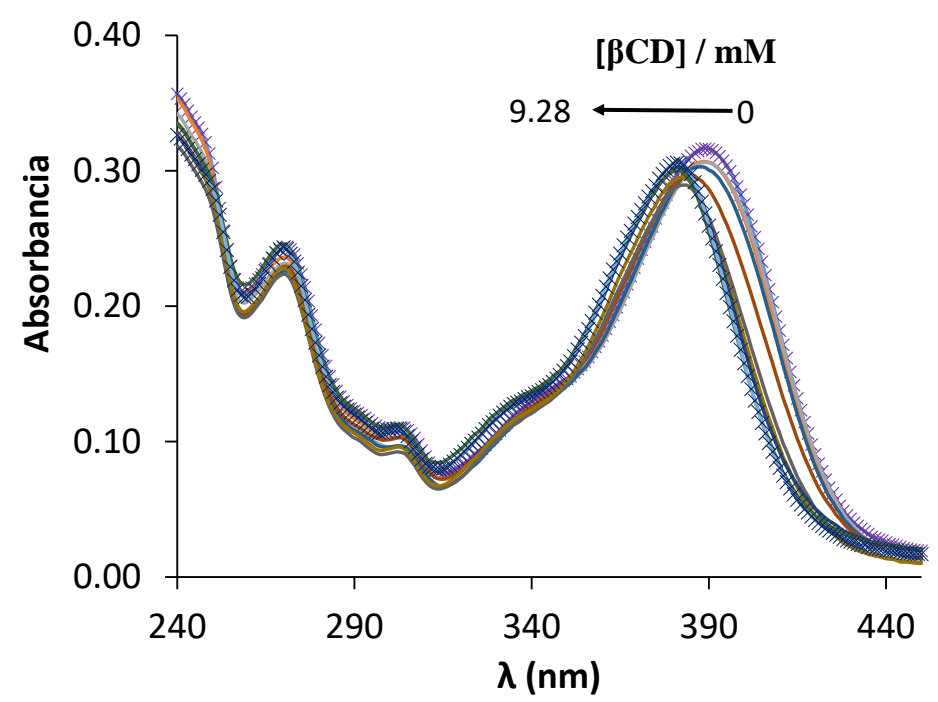

Figura 2.2.4. Conjunto de espectros de absorción en solución acuosa a un valor de pH de 8.6, medidos entre $240 \mathrm{~nm}$ - $450 \mathrm{~nm}$.y concentraciones de MGF' a $1 \times 10^{-2} \mathrm{mM}$ con diferentes concentraciones de $\beta-C D$ de $O \mathrm{M}$ hasta $9.28 \mathrm{mM}$.

Siguiendo con la metodología usada en Fluorescencia, se construye el gráfico de absorbancia en función del cociente de concentraciones de $[\beta C D] /[M G F ']$, 
verificando que este método es poco cuantitativo para terminar su relación molar (Figura 2.2.5).

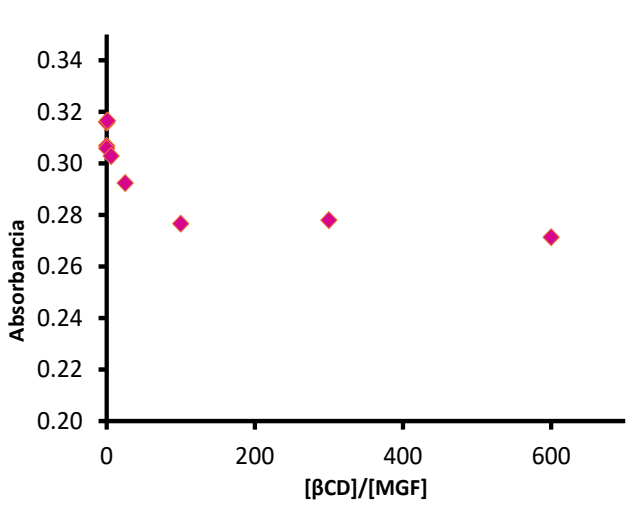

(a)

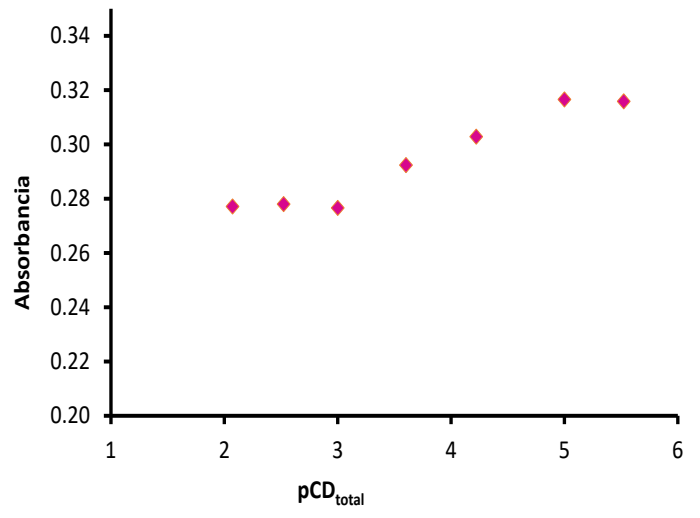

(b)

Figura 2.2.5. Gráfico de relaciones molares, donde en (a) se grafica absorbancia en función de $[\beta C D] /\left[M G F^{\prime}\right]$ y en (b) la curva de absorbancia en función de - $\log \left[\beta C D_{\text {total }}\right.$. Ambas curvas son para las soluciones de $\mathrm{pH}=8.6$ y a la longitud de onda de $388 \mathrm{~nm}$.

Para este caso también se observa que no es posible determinar la estequiometría con el método clásico de relaciones molares, pero es posible construir la gráfica de la Figura 2.2.5b.

Sin embargo, se puede realizar el análisis de componentes principales para determinar el número de especies presentes en el sistema que absorben diferente, alimentando el programa Minitab16 con el conjunto de espectros de absorción presentados en la Figura 2.2.4.

Tabla 2.2. Porcentaje de Varianza acumulada para la serie de espectros de Espectroscopía por UV-visible

\begin{tabular}{c|lllll} 
CP & $\mathbf{1}$ & $\mathbf{2}$ & $\mathbf{3}$ & $\mathbf{4}$ & $\mathbf{5}$ \\
\hline Porcentaje de & 75 & 95 & 99 & 99 & 100 \\
varianza acumulada & & & & &
\end{tabular}

En la Tabla 2.2 y en la Figura 2.2.6 se presentan los resultados más importantes del estudio de PCA, aplicados a los espectros de absorción UV-visible. 
En la Tabla 2.2 se muestra el porcentaje de varianza acumulada para cinco componentes, obteniéndose el $100 \%$ a partir del quinto componente. Pero al ver la gráfica de "loadings" del PCA, Figura 2.2.6, se observa que a partir del cuarto componente empieza a presentarse "ruido". Debido a esto es posible decir que hay al menos tres componentes que reconstruyen el $100 \%$ de la información.

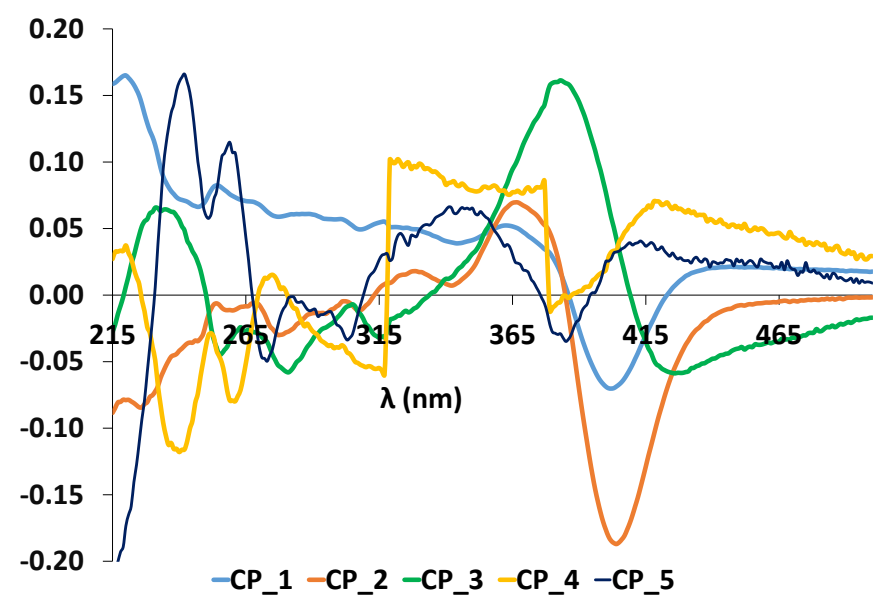

Figura 2.2.6. Gráfica de "loadings" del Análisis de Componentes Principales para espectros de absorción del sistema $M G F^{\prime}: \beta C D$ a un valor de $p H$ de 8.6.

Debido a que la información de la gráfica de "loadings" y la tabla 2.2, no es tan concluyente, porque el número de componentes que reconstruyen el $100 \%$ de la información relevante no coincide. Esto posiblemente se debe a que efecto de la $\beta$ $C D$ en los espectros de absorción es relativamente pequeño. Tomando en cuenta ambos criterios del PCA, es posible proponer de 3 a 5 especies que absorben diferente en el sistema.

\subsection{Determinación de constantes de formación y estequiometría}

Dado que el número de especies presentes con información espectroscópica diferente es más concluyente en los espectros de fluorescencia que en los espectros de absorción, se decidió usar el programa SQUAD (que son las siglas de Stability Quotients from Absorbance Data) ${ }^{39}$, para ajustar los espectros de fluorescencia y 
de absorción. Aunque el programa no fue diseñado para refinar constantes de equilibrio ajustando espectros de fluorescencia, en el apéndice $C$ se explica la razón por la cual esto es posible.

En SQUAD se consideró un equilibrio porque de acuerdo al estudio de PCA se obtuvieron 2 especies con diferente capacidad de fluorescencia, que es más concluyente que lo observado en absorción, para ajustar los espectros correspondientes. Debido a que no se conoce la estequiometria de dicho equilibrio se probaron las relaciones molares reportadas en trabajos para complejos tipo huésped - anfitrión, que se presentan en las Ecuaciones 2.1 a 2.3.

$$
\begin{aligned}
M G F^{\prime}+\beta C D & \rightarrow M G F^{\prime}(\beta C D) \\
M G F^{\prime}+2 \beta C D & \rightarrow M G F^{\prime}\left(\beta C D_{2}\right) \\
2 M G F^{\prime}+\beta C D & \rightarrow\left(M G F^{\prime}\right)_{2} \beta C D
\end{aligned}
$$

En la Tabla 2-3. se presentan los resultados obtenidos de log $K$ para cada equilibrio utilizado y para cada grupo de espectros, su correspondiente valor de incertidumbre (desviación estándar de la constante y desviación estándar de la regresión).

Como se puede observar en la Tabla 2.3, en el caso de los espectros de fluorescencia se puede concluir que el mejor modelo está dado por la formación del complejo $M G F^{\prime}(\beta C D)$, ya que presenta un valor de la desviación estándar de la regresión más bajo, tanto para los espectros de excitación y los de emisión. En el caso de los espectros de absorción el valor de la desviación estándar de la regresión para los tres casos, es del mismo orden de magnitud, como era de esperarse. Sin embargo el valor de log $\mathrm{K}$ obtenido por absorción para el complejo MGF'( $\beta C D)$ es muy similar al obtenido por fluorescencia, por lo que se concluye que este es el mejor modelo que explica todos los resultados experimentales, mostrados en este capítulo. 
Tabla 2.3. Modelos propuestos en el programa SQUAD para el cálculo de constantes de formación de MGF' con $\beta C D$.

\begin{tabular}{cccc}
\hline Modelo & Equilibrio formal & $\log \beta_{\mathrm{i}: \mathrm{j}}$ & $\sigma_{\text {reg }}$ \\
\hline \multicolumn{4}{c}{ Modelos para los espectros de excitación } \\
$\mathbf{1}$ & $M G F^{\prime}+\beta C D \rightarrow M G F^{\prime}(\beta C D)$ & $3.3747 \pm 0.0035$ & 6.880 \\
$\mathbf{2}$ & $M G F^{\prime}+2 \beta C D \rightarrow M G F^{\prime}(\beta C D)_{2}$ & $6.7957 \pm 0.0107$ & 17.27 \\
$\mathbf{3}$ & $2 M G F^{\prime}+\beta C D \rightarrow\left(M G F^{\prime}\right)_{2} \beta C D$ & $8.1678 \pm 0.0083$ & 11.79 \\
\hline \multicolumn{4}{c}{ Modelos para los espectros de emisión } \\
$\mathbf{1}$ & $M G F^{\prime}+\beta C D \rightarrow M G F^{\prime}(\beta C D)$ & $3.3702 \pm 0.0031$ & 6.959 \\
$\mathbf{2}$ & $M G F^{\prime}+2 \beta C D \rightarrow M G F^{\prime}(\beta C D)_{2}$ & $6.7924 \pm 0.0084$ & 15.63 \\
$\mathbf{3}$ & $2 M G F^{\prime}+\beta C D \rightarrow\left(M G F^{\prime}\right)_{2} \beta C D$ & $8.1599 \pm 0.0072$ & 11.79 \\
\hline \multicolumn{4}{c}{ Modelos para los espectros de absorción } \\
$\mathbf{1}$ & $M G F^{\prime}+\beta C D \rightarrow M G F^{\prime}(\beta C D)$ & $3.4129 \pm 0.0304$ & $6.542 \times 10^{-3}$ \\
$\mathbf{2}$ & $M G F^{\prime}+2 \beta C D \rightarrow M G F^{\prime}(\beta C D)_{2}$ & $7.0321 \pm 0.0377$ & $6.621 \times 10^{-3}$ \\
\hline
\end{tabular}

Posteriormente, se realizó un ajuste con la hoja de cálculo de Excel, cuya construcción se explica en el apéndice A, para las curvas de las relaciones molares, presentadas en las Figuras 2.2.2, y con los datos obtenidos por SQUAD para una relación molar de $1 \mathrm{MGF}^{\prime}: 1 \beta \mathrm{CD}$. 


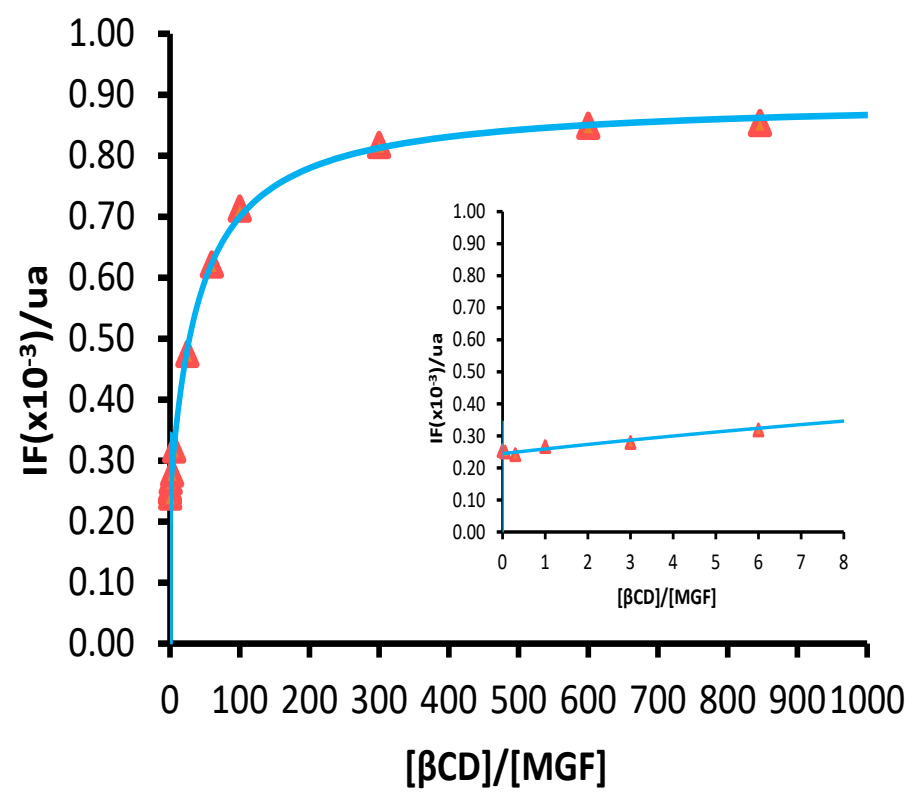

(a)

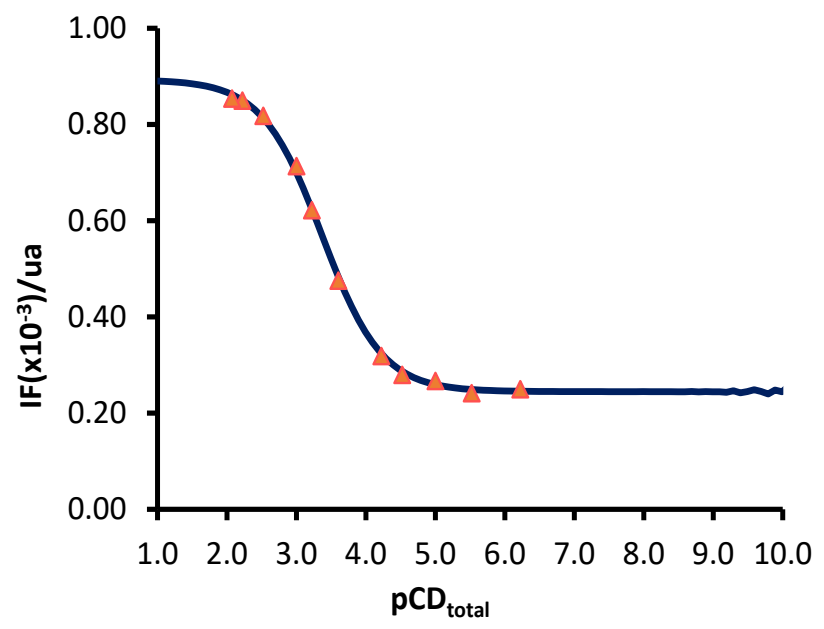

(b)

Figura 2.2.7. Curvas de Intensidad de Fluorescencia (IF) marcadores representan los datos experimentales y la línea continua el ajuste con los datos refinados por SQUAD para el complejo $M G F^{\prime}: \beta C D$. Donde el (a) representa la curva de relaciones molares típicas, el inserto es la ampliación de la curva para las relaciones molares de 0 a 8 y el (b) muestra el máximo de IF como función de $p C D_{\text {total, }} p C D_{\text {total }}=-\log \left[C D_{\text {total }}\right]$ de $\beta-C D$, para los espectros de excitación y de emisión, a un valor de $\mathrm{pH}=8.6$.

Los ajustes para los espectros de fluorescencia se muestran en la Figura 2.2.7, utilizando los resultados de la Tabla 2.4 y los factores de respuesta 
correspondientes a las longitudes de onda de los máximos de excitación y de emisión.

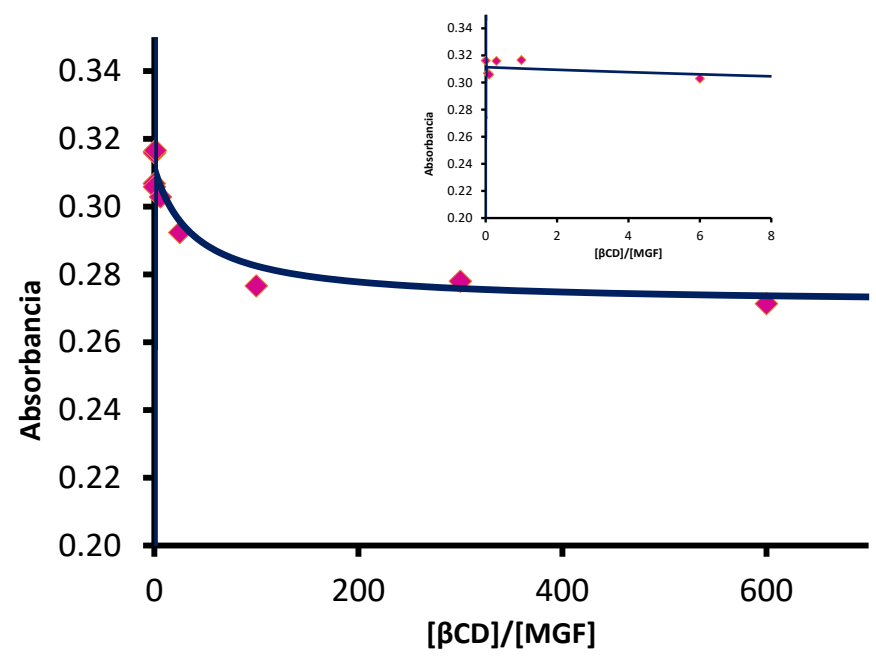

(a)

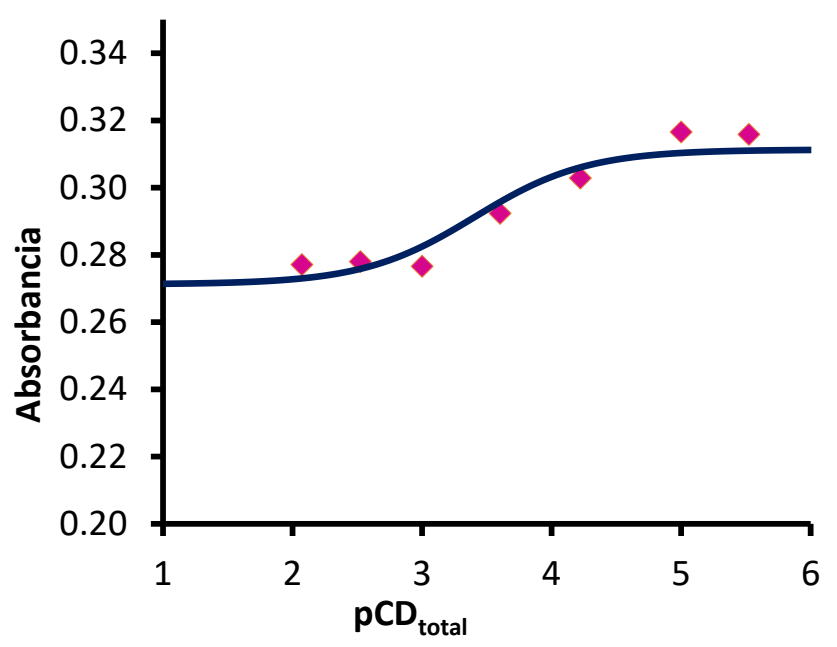

(b)

Figura 2.2.8. Gráfica de absorbancia en función de menos el logaritmo de la concentración total $\beta C D$, tomados a un valor de $p H=8.6$, donde los marcadores representan los datos experimentales y la línea continua el ajuste con el valor de logß1:1 obtenido por SQUAD, a $388 \mathrm{~nm}$.

Los ajustes para los espectros de absorción se muestran en la Figura 2.2.8, utilizando los resultados de la Tabla 2.4 y los coeficientes de absortividad molar correspondientes a las longitudes de onda de $388 \mathrm{~nm}$. 
A pesar de que la reacción de formación del complejo de inclusión es muy poco cuantitativa, es posible realizar buenos de las curvas de relaciones molares con los valores de las constantes de inclusión obtenidas por SQUAD, para el modelo propuesto de un equilibrio con relación molar 1:1.

Tabla 2.4. Valores de constantes de formación de la especie MGF'( $\beta C D)$.

\begin{tabular}{c|c|c|c}
\hline \multicolumn{2}{c|}{ Técnica } & $\log \beta_{1: 1} \pm \sigma_{\text {const }^{*}}$ & $\sigma_{\text {reg }}{ }^{* *}$ \\
\cline { 1 - 1 } \multicolumn{2}{c|}{ Espectroscopía UV-visible } & $3.413 \pm 0.030$ & $6.542 \times 10^{-3}$ \\
\cline { 1 - 1 } Fluorescencia & Excitación & $3.375 \pm 0.004$ & $6.880 \times 10^{-3}$ \\
& Emisión & $3.370 \pm 0.003$ & $6.960 \times 10^{-3}$ \\
${ }^{*} \sigma$ const= desviación estándar sobre la constante & \\
${ }^{* *} \sigma_{\text {tot }}=$ desviación estándar total sobre las constantes &
\end{tabular}




\subsection{Conclusiones}

Se realizaron estudios espectrofotométricos UV-visible y Fluorescencia para la formación de complejos de inclusión entre $M G F^{\prime}(\beta C D)$ en medio acuoso a un pH 8.6. Los valores encontrados a través de ambos métodos fueron muy similares, lo que enfatiza la confiabilidad de las constantes de complejación. Además, ambos

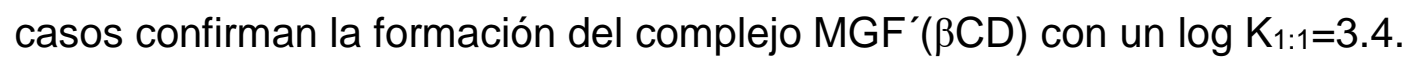

Como se mencionó al inicio a un pH de 8.6 la especie que está presente con una mayor fracción molar es $\mathrm{H}_{2} \mathrm{MGF}^{2-}$ o también nombrada en este capítulo como MGF', presenta poca Fluorescencia según los experimentos realizados, pero al estar incluida en la cavidad de ciclodextrina muestra un efecto en el aumento de la intensidad de Fluorescencia como se mostró en los espectros de emisión y excitación al realizar adiciones de $\beta C D$ a una cantidad fija de MGF'.

Debido a su alta sensibilidad, selectividad y simplicidad instrumental, el método de Fluorescencia se utilizó para investigar los fenómenos de complejos de inclusión y determinar las constantes de formación con valores de $3.413 \pm 0.030,3.375 \pm 0.004$, $3.370 \pm 0.003$, por Espectroscopía UV-Visible, emisión y excitación por Fluorescencia; respectivamente. 


\section{CAPÍTULO 3}

\section{DETERMINACIÓN DE LAS CONSTANTES DE ACIDEZ CONDICIONALES POR ESPECTROFOTOMETRÍA UV-visible PARA MANGIFERINA EN PRESENCIA DE $\beta$ - CICLODEXTRINA}




\section{CAPÍTULO 3}

\section{DETERMINACIÓN DE LAS CONSTANTES DE ACIDEZ CONDICIONALES POR ESPECTROFOTOMETRÍA UV-visible PARA MANGIFERINA EN PRESENCIA DE $\beta$-CICLODEXTRINA}

La determinación de las constantes de acidez condicionales de MGF' en presencia de $\beta C D$, se llevó a cabo en solución acuosa, donde se hizo un estudio espectrofotométrico UV-Visible por medio de cambios de $\mathrm{pH}$ desde valores ácidos hacia basicos, para evidenciar la interacción entre la $\beta$-ciclodextrina y la mangiferina, manteniendo fijas las concentraciones de ambos compuestos.

\subsection{Preparación de soluciones}

Las concentraciones de usadas en este capítulo para la determinación de constantes de acidez condicionales fueron para mangiferina $4.8 \times 10^{-5} \mathrm{M}$ y para $\beta$ ciclodrextrina se utilizaron diferentes niveles de concentración: $1 \mathrm{mM}$ y $10 \mathrm{mM}$. Los experimentos se llevaron a cabo a temperatura constante de $25.0 \pm 0.5^{\circ} \mathrm{C}$, en medio acuoso.

\subsubsection{Procedimiento experimental}

Para la valoración se utiliza una solución de $\mathrm{HCl}$ para ajustar el pH inicial en 3.6, posteriormente se fue adicionando diferentes volúmenes de una solución concentrada de $\mathrm{NaOH}$, variando el $\mathrm{pH}$ con cambios no mayores de 0.3 unidades, en un intervalo de $3.6 \leq \mathrm{pH} \leq 12.5$. La mezcla fue colocada en un baño térmico con una celda termostatada a una temperatura de $25.0 \pm 0.5^{\circ} \mathrm{C}$ mediante el uso de un termorregulador Lauda Brinkmann ecoline RE 106, manteniendo en agitación 
constante. Para cada cambio de pH se registró la medición del espectro de absorción dentro de un intervalo de 240 a $500 \mathrm{~nm}$.

Los espectros de absorción se determinaron con un espectrofotómetro Perkin-Elmer Lambda 950. Las muestras se colocaron en una celda de cuarzo con una longitud de paso óptico de $1.0 \mathrm{~cm}$, usando como blanco agua y así generar la línea base.

Las mediciones de $\mathrm{pH}$ se hicieron con un potenciómetro $\mathrm{HACH}$ modelo sensION+ pH31 equipado con un electrodo combinado de vidrio con referencia externa de $\mathrm{Ag} / \mathrm{AgCl}$, que permite medir valores de $\mathrm{pH}$ desde 0 hasta 14 , el electrodo fue calibrado con un buffer a un valor de $\mathrm{pH} 7$.

En el análisis de resultados se corrigieron los valores de concentración según el efecto de dilución alcanzado, aunque ésta se cuida que sea $\leq 10 \%$ del volumen total de la solución; los valores de pH también son corregidos de acuerdo a la siguiente ecuación ${ }^{40}$ :

$$
p H_{c o r r}=p H_{\text {exp }}+\left(\frac{p H_{c a l}-p H_{\text {exp }}}{p H_{c a l}}\right) E f
$$

donde el $\mathrm{pH}_{\text {corr }}=\mathrm{pH}$ corregido, $\mathrm{pH}_{\exp }=\mathrm{pH}$ medido experimentalmente, $\mathrm{pH}_{c a l}=\mathrm{pH}$ de calibración del electrodo de vidrio, $E f=$ Eficiencia electromotriz del electrodo de vidrio.

Con el programa computacional SQUAD se obtuvieron las constantes de acidez condicionales, para ser utilizadas en el ajuste de absorbancia como función del pH a diferentes longitudes de onda.

El ajuste se llevó a cabo de la siguiente manera:

Se construye un modelo en una hoja de Excel que toma en cuenta las fracciones molares como pesos estadísticos de la absorbancia y los coeficientes de absortividad molar obtenidos por SQUAD.

La Espectroscopía requiere de las leyes de Beer y de aditividad para determinar la absorbancia de las especies químicas presentes. Donde la respuesta a una 
perturbación presenta un comportamiento lineal con respecto a las concentraciones de las especies que lo producen. Debido a esto es posible predecir los valores de constantes de equilibrio utilizando estas leyes, graficando la respuesta (absorbancia) como función de la longitud de onda para diferentes concentraciones de las especies del sistema que presentan absorción de luz.

\subsection{Resultados y discusión}

Si la $\beta$-ciclodextrina interacciona con la mangiferina, un aumento en la concentración de la $\beta$-ciclodextrina debe afectar a las propiedades ácido - base de las especies de la mangiferina.

Si la $\beta C D$ se encuentra en mucho mayor concentración que la mangiferina, se puede decir que la concentración de $\beta C D$ es constante en el sistema. Es entonces que se pueden definir especies generalizadas a través de la interacción con $\beta C D$ y relacionarlas por medio de equilibrios generalizados cuyas constantes condicionales sólo dependen de la concentración de $\beta C D$, como se demuestra en el método de especies y equilibrios generalizados ${ }^{41}$.

Para este trabajo y en este capítulo se pueden definir las especies generalizadas de la mangiferina en las ecuaciones 3.2 a la 3.6.

$$
\begin{aligned}
& H_{4} M G F^{\prime}=H_{4} M G F+H_{4} M G F(\beta C D)+H_{4} M G F(\beta C D)_{2}+\ldots \\
& H_{3} M G F^{\prime}=H_{3} M G F^{-}+H_{3} M G F(\beta C D)^{-}+H_{3} M G F(\beta C D)_{2}^{-}+\ldots \\
& H_{2} M G F^{\prime}=H_{2} M G F^{2-}+H_{2} M G F(\beta C D)^{2-}+H_{2} M G F(\beta C D)_{2}^{2-}+\ldots \\
& H M G F^{\prime}=H M G F^{3-}+H M G F(\beta C D)^{3-}+H M G F(\beta C D)_{2}^{3-}+\ldots \\
& M G F^{\prime}=M G F^{4-}+M G F(\beta C D)^{4-}+M G F(\beta C D)_{2}^{4-}+\ldots
\end{aligned}
$$


Estas especies generalizadas pueden intercambiar protones a través, por ejemplo, de los equilibrios generalizados de acidez, descritos en las ecuaciones (3.7) a la (3.10).

$$
\begin{gathered}
H_{4} M G F^{\prime}=H_{3} M G F^{\prime}+H^{+} \quad \text { con } \quad K_{a 1}^{\prime} \equiv \frac{\left[H_{3} M G F^{\prime}\right]\left[H^{+}\right]}{\left[H_{4} M G F^{\prime}\right]} \\
H_{3} M G F^{\prime}=H_{2} M G F^{\prime}+H^{+} \text {con } \quad K_{a 2}^{\prime} \equiv \frac{\left[H_{2} M G F^{\prime}\right]\left[H^{+}\right]}{\left[H_{3} M G F^{\prime}\right]} \\
H_{2} M G F^{\prime}=H M G F^{\prime}+H^{+} \text {con } \quad K_{a 3}^{\prime} \equiv \frac{\left[H M G F^{\prime}\right]\left[H^{+}\right]}{\left[H_{2} M G F^{\prime}\right]} \\
H M G F^{\prime}=M G F^{\prime}+H^{+} \quad \text { con } \quad K_{a 4}^{\prime} \equiv \frac{\left[M G F^{\prime}\right]\left[H^{+}\right]}{\left[H M G F^{\prime}\right]}
\end{gathered}
$$

En el apéndice $D$ se muestra cómo las constantes de mangiferina dependen de la concentración de $\beta C D$.

Para estudiar el efecto de la $\beta C D$ sobre la mangiferina, en los experimentos descritos en este capítulo se utilizaron dos diferentes concentraciones de $\beta C D$ : $1 \mathrm{mM}$ y $10 \mathrm{mM}$.

\subsubsection{Determinación de espectros de absorción de la mangiferina con $[\beta C D]=1 \mathrm{mM}$}

Para la determinación de las constantes condicionales de acidez se registraron los espectros obtenidos por las mediciones realizadas por Espectroscopía UV-visible, obteniendo para la valoración ácido-base un mínimo de 54 espectros de absorción, algunos de los cuales se muestran en la Figura 3.2.1, con el objeto de mostrar el comportamiento espectral dentro del intervalo de $\mathrm{pH}$ de interés. 


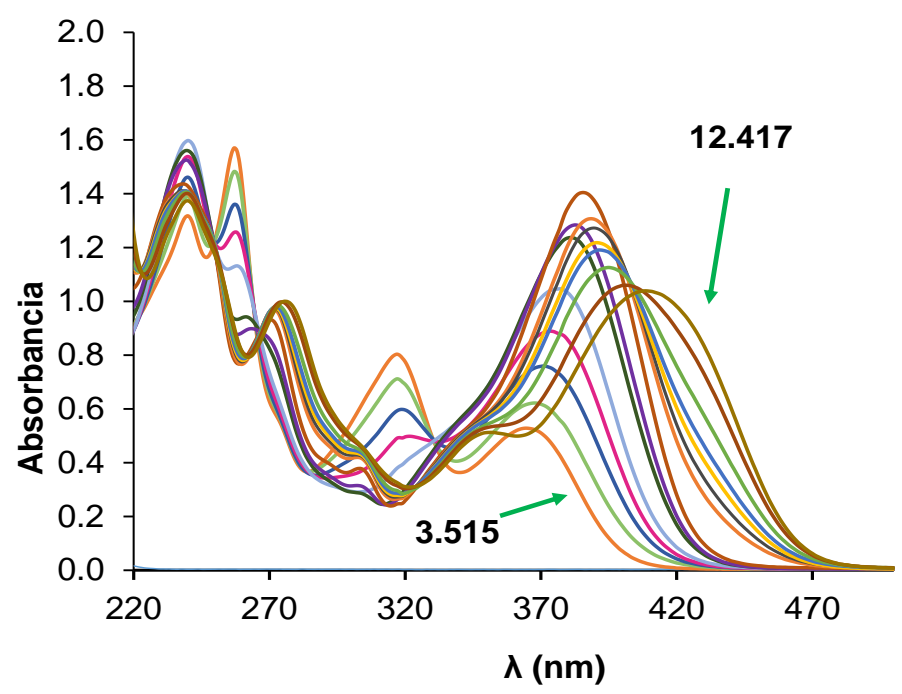

Figura 3.2.1. Espectros de absorción de la valoración para la interacción de mangiferina $4.8 \times 10^{-5} \mathrm{M}$ y $\beta-C D 1 \mathrm{mM}$, dentro de un intervalo de $3.515 \leq \mathrm{pH} \leq 12.417$.

En estos resultados no se observó ningún cambio espectral entre $3.5 \leq \mathrm{pH} \leq 5.0$, además en el intervalo de $500 \mathrm{~nm} \leq \lambda \leq 750 \mathrm{~nm}$ la absorbancia es cero. Se hace notar la presencia de 4 puntos isosbésticos en 250, 268, 320 y $409 \mathrm{~nm}$ indicando la presencia de los equilibrios ácido-base y cuatro bandas de absorbancia máxima representativas en $244,258,320$ y $367 \mathrm{~nm}$ para los espectros medidos en valores de $\mathrm{pH}$ ácidos.

A medida que el $\mathrm{pH}$ aumenta, los máximos relevantes presentan un efecto hipercrómico y batocrómico, la banda inicial en $367 \mathrm{~nm}$ se va recorriendo a longitudes de onda más grandes, observándose una coloración amarilla que se va incrementando en la solución conforme aumenta el pH.

Con la información de los resultados de los espectros de absorción se construyen las curvas de absorbancia en función del pH que se presentan en la Figura 3.2.2, para cada longitud de onda de las bandas principales en medio ácido. 


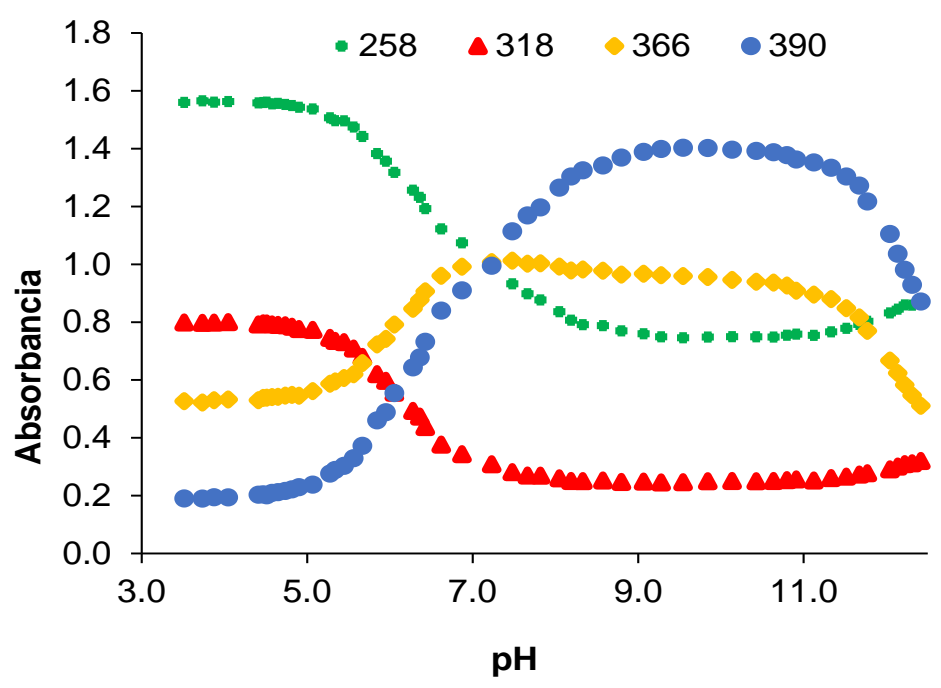

Figura 3.2.2. Gráficas de Absorbancia en función del $\mathrm{pH}$, a partir de la valoración de mangiferina en presencia de $\beta C D 1 \mathrm{mM}$. A los valores de longitud de onda representativos en $\lambda_{\text {máx }}=258(\circ), 318$ $(\mathbf{\Delta}), 366(\diamond), 390(\bullet) \mathrm{nm}$.

En la Figura 3.2.2, se construyen los gráficos de absorbancia en función del pH, para las bandas de absorción que presentan máximos a una longitud de onda específica. Dichas curvas presentan tendencias sigmoidales, lo cual es indicativo de la presencia de varios equilibrios generalizados de acidez para las diferentes especies de mangiferina, $a[\beta C D]=1 \mathrm{mM}$; además cada cambio de pendiente 0 punto de inflexión indica la aproximación para estimar los valores de las constantes condicionales de acidez.

\subsubsection{Determinación de constantes de acidez condicionales de la mangiferina con el programa SQUAD para $[\beta C D]=1 \mathrm{mM}$}

A partir de los resultados espectrofotométricos y los valores de $\mathrm{pH}$ correspondientes se alimenta el programa computacional SQUAD para ejecutar el refinamiento de las constantes condicionales de acidez. 
Experimentalmente se obtienen 54 espectros de absorción por cada valor de $\mathrm{pH}$ corregido con la ecuación 3.1 y medidos dentro del intervalo de longitudes de onda de 240 - $500 \mathrm{~nm}$ con incrementos de $2 \mathrm{~nm}$, con esta información se alimenta el software SQUAD, considerando un modelo químico con cuatro equilibrios generalizados de formación global de $\mathrm{MGF}^{\prime}$ con $\mathrm{H}^{+}$.

Los resultados obtenidos para las constantes condicionales de formación se muestran en la Tabla 3.1, con su valor en la desviación estándar.

Tabla 3.1. Constantes condicionales de formación globales de la mangiferina en presencia de $\beta$-ciclodextrina $1 \mathrm{mM}$ obtenidas con el programa SQUAD.

\begin{tabular}{lc}
$\begin{array}{c}\text { Equilibrio generalizado } \\
\text { de formación global }\end{array}$ & $\log \beta_{i}^{\prime} \pm \sigma_{\text {const }}$ \\
\hline$M G F^{\prime}+H^{+}=H M G F^{\prime}$ & $\log \beta_{1}^{\prime}=12.420 \pm 0.048$ \\
$M G F^{\prime}+2 H^{+}=H_{2} M G F^{\prime}$ & $\log \beta_{2}^{\prime}=21.608 \pm 0.047$ \\
$M G F^{\prime}+3 H^{+}=H_{3} M G F^{\prime}$ & $\log \beta_{3}^{\prime}=29.236 \pm 0.043$ \\
$M G F^{\prime}+4 H^{+}=H_{4} M G F^{\prime}$ & $\log \beta_{4}^{\prime}=35.363 \pm 0.004$ \\
\hline $\begin{array}{l}\sigma_{\text {const }}=\text { desviación estándar sobre la constante; } \\
\sigma_{\text {tot }}=\text { desviación estándar total de la regresión }=8.58 \times 10^{-3}\end{array}$
\end{tabular}

Por otra parte, en la Figura 3.2.3 se muestran los coeficientes de absortividad molar obtenidos por SQUAD con sus respectivas desviaciones estándar para las 5 especies generalizadas de mangiferina en presencia de $\beta$-ciclodextrina, por ejemplo para la especie MGF' se observan 5 máximos representativos en 242, 254, 282, 346 y 424 nm, así como también la formación de puntos isosbésticos. 


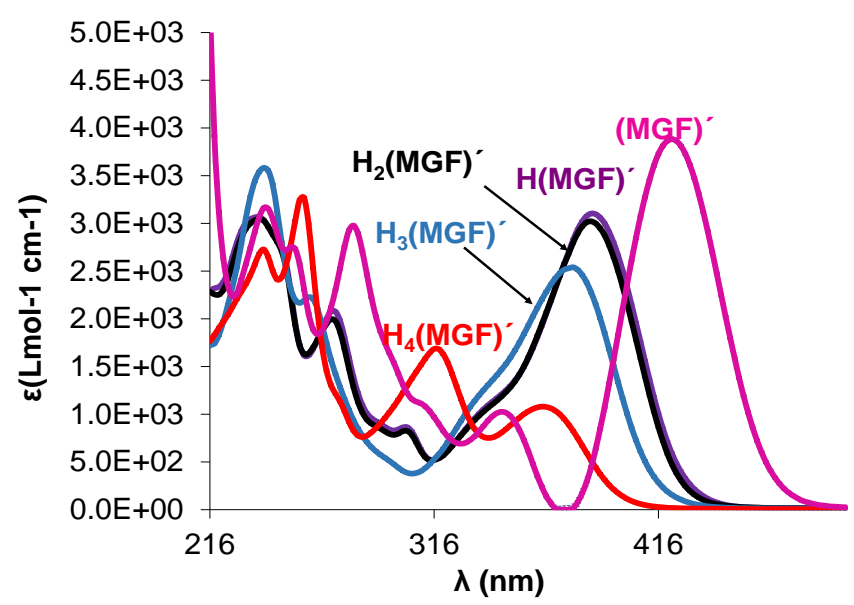

Figura 3.2.3 Coeficientes de absortividad molar de las diferentes especies de mangiferina $\left(4.8 \times 10^{-5} \mathrm{M}\right)$ en presencia de $\beta$-ciclodextrina $(1 \mathrm{mM})$ en el intervalo de $3.515 \leq \mathrm{pH} \leq 12.417$.

\subsubsection{Comparación de los resultados para la mangiferina en ausencia de $\beta C D$ y para $[\beta C D]=1 \mathrm{mM}$}

Mediante el uso de una hoja de cálculo de Excel se construyó el modelo teórico que toma en cuenta las fracciones molares de cada especie generalizada de mangiferina, sus coeficientes de absortividad molar (determinados con SQUAD), las concentraciones totales de los componentes, las leyes de Beer y de aditividad. Esto permite analizar si posible ajustar los datos experimentales con el modelo refinado por SQUAD.

En la Figura 3.2.4 se grafica la absorbancia en función del pH a las diferentes longitudes de onda representativas en el conjunto de espectros de absorción de la Figura 3.2.1. El ajuste del modelo a los datos se representa con la línea continua.

Esto confirma que, el modelo propuesto en SQUAD, de 5 especies generalizadas y 4 valores de pKa's sigue la misma tendencia que la tendencia observada en los experimentos, pero que en valores de $\mathrm{pH}$ mayores de 8 presenta una mayor desviación en algunas longitudes de onda. Esto demuestra la formación de los complejos de inclusión con diferentes especies de la mangiferina y la $\beta C D$. 

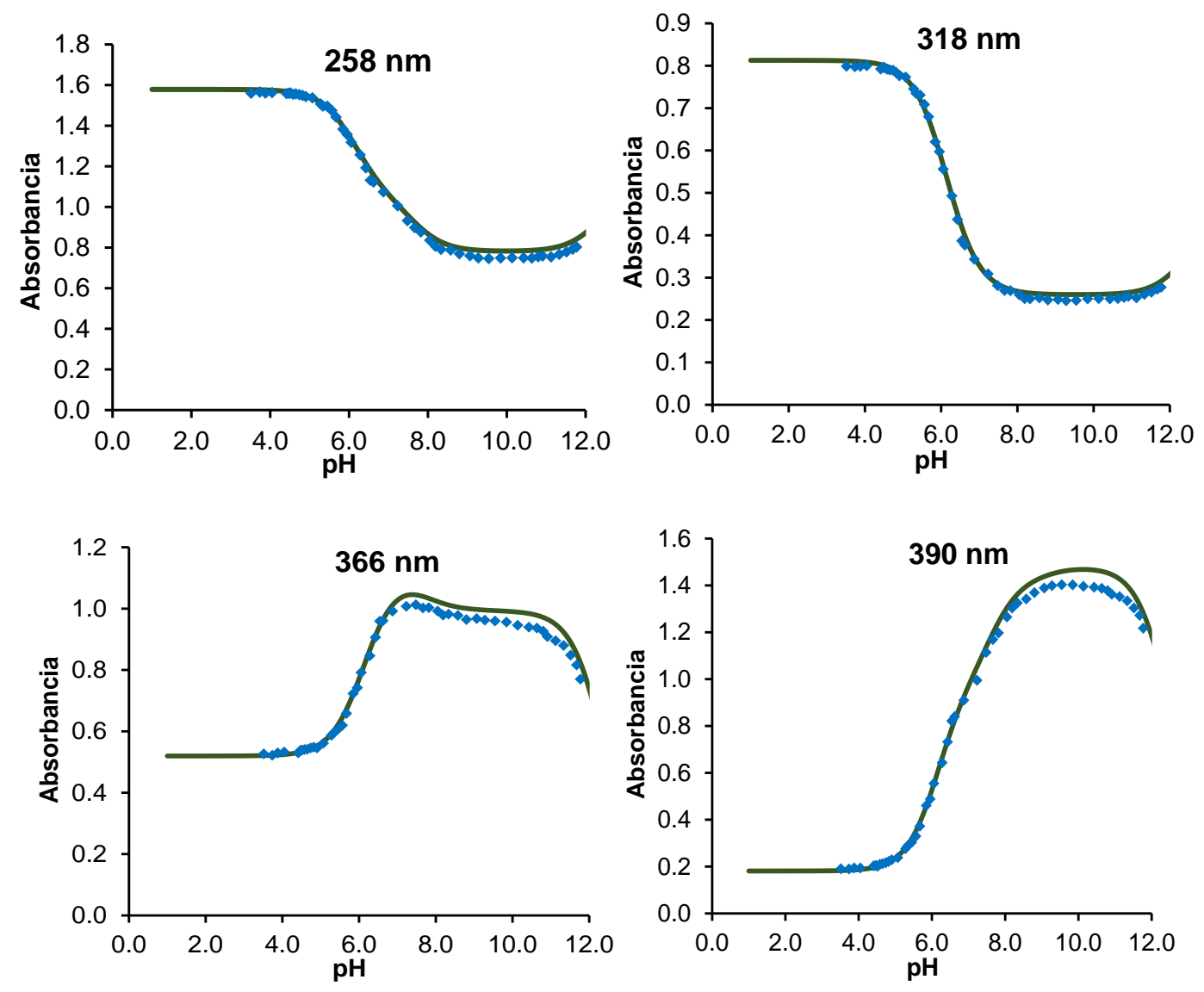

Figura 3.2.4. Ajuste de las curvas de absorbancia en función del valor de $\mathrm{pH}$ para diferentes longitudes de onda (258nm, 318nm 366nm y 390nm). Los marcadores representan los datos experimentales y la línea continua indica el ajuste con el modelo.

De acuerdo a Gómez-Zaleta y col., los valores de pKa para las diferentes especies de la mangiferina son: $\mathrm{pK}_{\mathrm{a} 1}=6.52 \pm 0.06, \mathrm{pK}_{\mathrm{a} 2}=7.97 \pm 0.06, \mathrm{pK}_{\mathrm{a} 3}=9.44 \pm 0.04$, $\mathrm{pK}_{\mathrm{a} 4}=12.10 \pm 0.01$. Estos datos se comparan con los valores obtenidos de $\mathrm{pKa}$ condicionales, en presencia de $\beta C D 1 \mathrm{mM}$ (ver Tabla 3.2.) En el apéndice $\mathrm{E}$ se explica cómo es que se obtienen estos valores a partir de los presentados en la Tabla 3.1. 
Tabla 3.2. Valores de pKa de especies de mangiferina y de pKa' de especies generalizadas de mangiferina en presencia de $\beta$-ciclodextrina $1 \mathrm{mM}$.

\begin{tabular}{ccc}
\hline $\begin{array}{c}\text { Equilibrio generalizado de } \\
\text { acidez }\end{array}$ & Valor reportado ${ }^{12}$ & Experimental \\
\hline$H_{4} M G F^{\prime}=H_{3} M G F^{\prime}+H^{+}$ & $\mathrm{pK}_{\mathrm{a} 1=6.52 \pm 0.06}$ & $\mathrm{pK}_{\mathrm{a} 1}=6.127 \pm 0.043$ \\
$H_{3} M G F^{\prime}=H_{2} M G F^{\prime}+H^{+}$ & $\mathrm{pK}_{\mathrm{a} 2=7.97 \pm 0.06}$ & $\mathrm{pK}_{\mathrm{a} 2}^{\prime}=7.629 \pm 0.064$ \\
$H_{2} M G F^{\prime}=H M G F^{\prime}+H^{+}$ & $\mathrm{pK}_{\mathrm{a} 3=9.44 \pm 0.04}$ & $\mathrm{pK}_{\mathrm{a} 3}^{\prime}=9.187 \pm 0.067$ \\
$H M G F^{\prime}=M G F^{\prime}+H^{+}$ & $\mathrm{pK}_{\mathrm{a} 4=12.10 \pm 0.01}$ & $\mathrm{pK}_{\mathrm{a} 4}^{\prime}=12.421 \pm 0.048$ \\
\hline
\end{tabular}

Para poder hacer una comparación más clara que la que puede hacerse al analizar solamente los valores que se presentan en la tabla 3.2, se muestra el diagrama de distribución de las especies de mangiferina como función del pH, en la Figura 3.2.5. En este diagrama se grafican las fracciones molares de las especies de mangiferina $\left(f_{i}\right)$ como una función del $p H$. Una discusión acerca de la construcción e interpretación de este diagrama de distribución se discute en el apéndice F.

En la Tabla 3.2 y en el diagrama de la Figura 3.2.5. se observa que las los valores de $\mathrm{pK}^{\prime}{ }_{\mathrm{a} 1}, \mathrm{pK}_{\mathrm{a} 2}^{\prime}$ y $\mathrm{pK}_{\mathrm{a} 3}^{\prime}, \mathrm{a}[\beta \mathrm{CD}]=1 \mathrm{mM}$, se hacen aproximadamente 0.3 unidades más pequeños que los valores correspondientes (en ausencia de $\beta C D$ ). Pero ocurre lo contrario al valor de $\mathrm{pK}^{\prime}{ }_{\mathrm{a} 4}$, con respecto al valor de $\mathrm{pK}_{\mathrm{a} 4}$. Esto podría deberse a que los complejos de inclusión de $\beta C D$ con las especies $\mathrm{H}_{3} \mathrm{MGF}^{-}, \mathrm{H}_{2} \mathrm{MGF}^{2-}$, $\mathrm{HMGF}^{3-}$, son más estables que con las especies $\mathrm{H}_{4} \mathrm{MGF}$, MGF${ }^{4-}$.

Tal vez el cambio más notorio en la Figura 3.2.5 es que la especie HMGF' aumenta su zona de predominio en presencia de $\beta C D 1 \mathrm{mM}$ con respecto al que presenta cuando no hay $\beta C D$ en el sistema. 


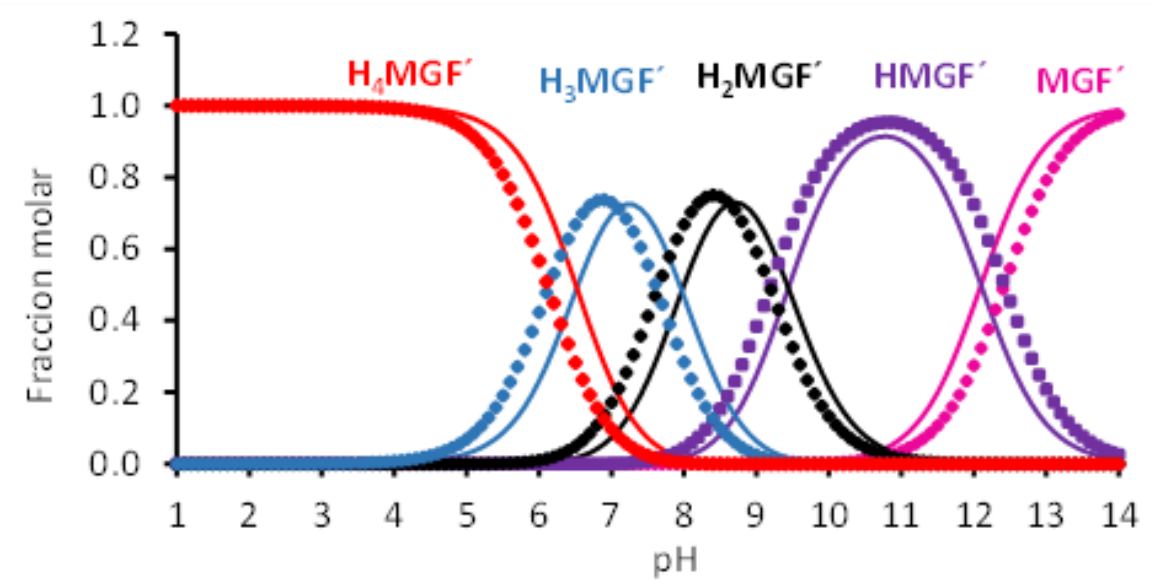

Figura 3.2.5. Diagrama de distribución de especies, para sistemas de mangiferina.

En línea continua se representan las especies correspondientes al sistema de la mangiferina, en tanto que las fracciones del sistema de la mangiferina en presencia de la $\beta C D$ se identifican por los marcadores. En (rojo) se representa $H_{4} M G F^{\prime}$, en (azul) $H_{3} M G F^{\prime}$, en (negro) $H_{2} M G F^{\prime}$, en (violeta) HMGF' y en (magenta) MGF'.

Se efectuó una comparación de los coeficientes de absortividad molar de cada especie de la mangiferina. El Figura 3.2.6. se muestran las curvas de los coeficientes de absortividad molar donde se observa que las especies $\mathrm{H}_{4} \mathrm{MGF}$, $\mathrm{H}_{3} \mathrm{MGF}^{\prime}, \mathrm{H}_{2} \mathrm{MGF}^{\prime}$ y $\mathrm{HMGF}^{\prime}$ no tienen cambios significativos con respecto a los coeficientes de las especies $\sin \beta C D$. La especie MGF' es la que presenta un gran aumento en el coeficiente de absortividad molar. Este comportamiento se puede deber a que el complejo de inclusión de MGF4- con $\beta C D$ muestre interacciones específicas sobre el grupo cromóforo, y que esto no ocurra así para las otras especies de mangiferina. 

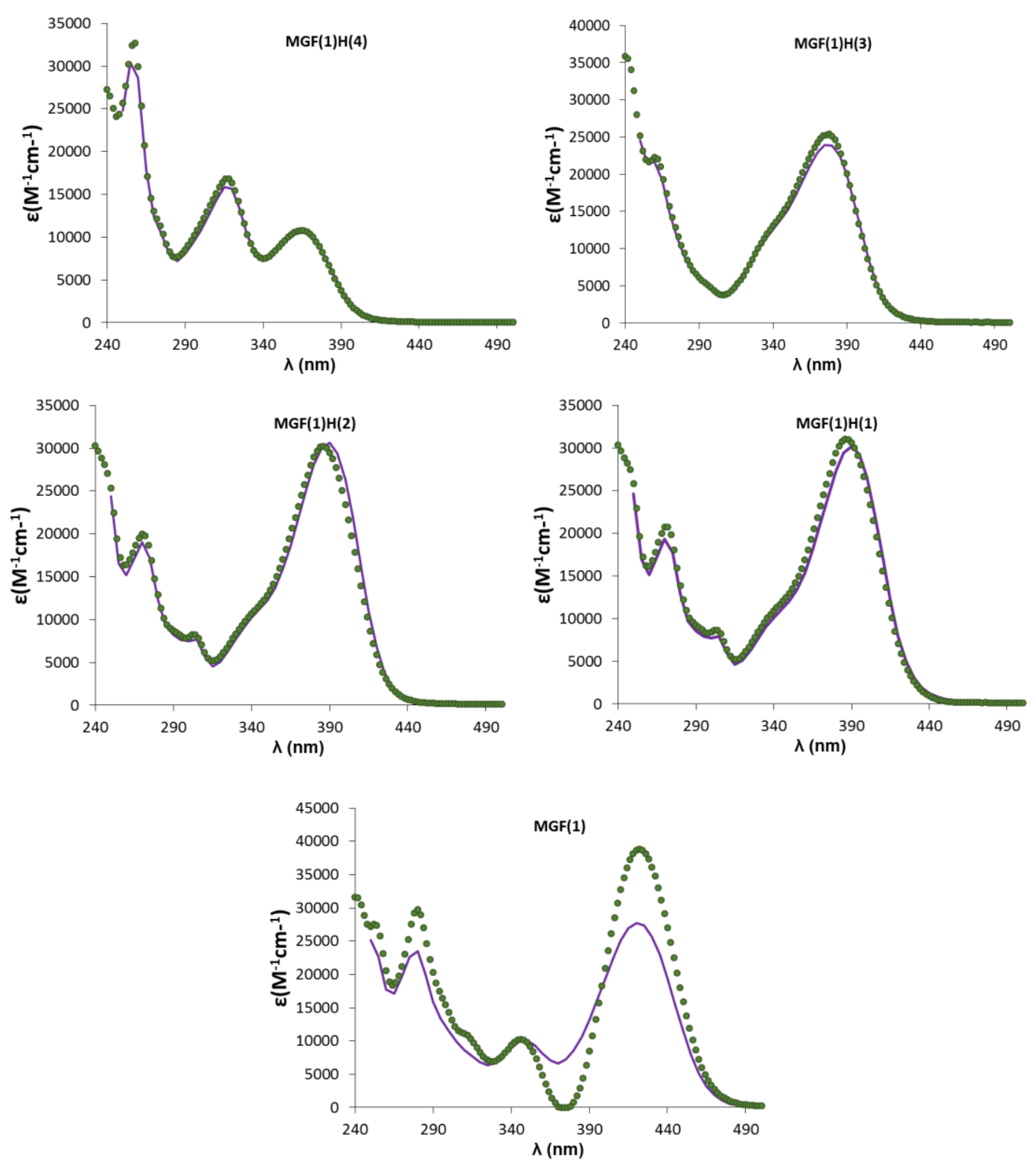

Figura 3.2.6. Coeficientes de absortividad molar obtenidos por $S Q U A D$ en la variación de $p H$, para las especies de mangiferina $\sin \beta C D$ (línea continua) y las especies generalizadas de mangiferina, para $[\beta C D]=1 \mathrm{mM}$ (marcadores). 
Para verificar que las constantes condicionales de acidez de mangiferina dependen de la concentración de $\beta$-ciclodextrina, se realizó una segunda prueba a las mismas condiciones de concentración para la mangiferina $\left(4 \times 10^{-5} \mathrm{M}\right)$, únicamente aumentando la concentración de $\beta C D$ a $10 \mathrm{mM}$, pero siguiendo la metodología del experimento anterior. Los espectros de absorción determinados en estos experimentos se muestran en la Figura 3.2.7. Se adquirieron 45 espectros de absorción, dentro de un intervalo de $\mathrm{pH}$ de $3.586 \leq \mathrm{pH} \leq 12.326$ y donde se observa que a la longitud de onda de $435 \mathrm{~nm}$ aparece un hombro, mismo que se puede relacionar con la formación de la especie completamente desprotonada $\mathrm{MGF}^{4-}$, a valores más grandes de $\mathrm{pH}$ que para $[\beta C D]=1 \mathrm{mM}$ (ver Figura 3.2.1) o en ausencia de $\beta C D$.

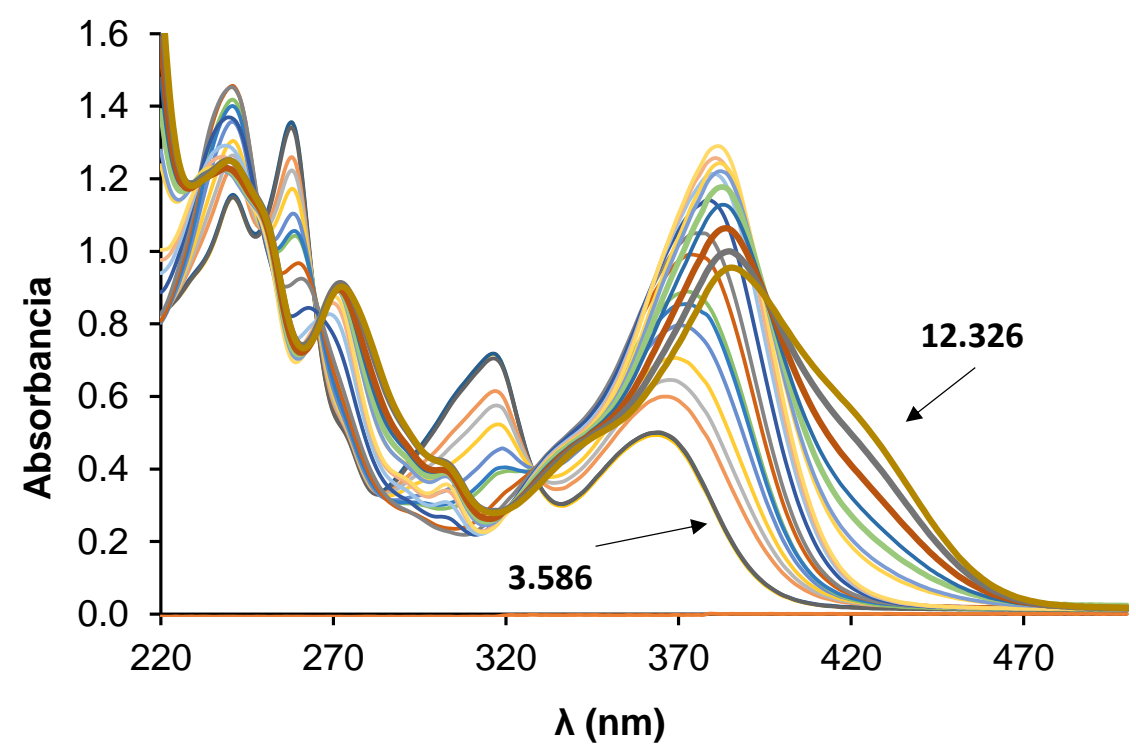

Figura 3.2.7. Espectros de absorción para la valoración entre la interacción entre mangiferina $\left(4.0 \times 10^{-5} \mathrm{M}\right)$ y $\beta-C D(10 \mathrm{mM})$, medidos dentro del intervalo de $3.586 \leq \mathrm{pH} \leq 12.326$. 
En estos experimentos tampoco se observó cambio espectral entre $3.5 \leq \mathrm{pH} \leq 5.0$. Se hace notar la presencia de cuatro bandas de absorbancia máxima representativas en 242, 258, 318 y $366 \mathrm{~nm}$ para los espectros obtenidos en valores de $\mathrm{pH}$ ácidos.

A medida que el $\mathrm{pH}$ aumenta, los máximos relevantes presentan un efecto hipercrómico seguido de un corrimiento batocrómico, principalmente para la banda inicial en $367 \mathrm{~nm}$ hasta llegar a un $\mathrm{pH} \approx 9.5$, para seguir con la disminución en la absorbancia hasta valores muy básicos de $\mathrm{pH}$.

En la Figura 3.2.8 se muestran las curvas de absorbancia en función del pH, tomando en cuenta las longitudes de onda significativas en los valores máximos de absorbancia para el conjunto de espectros de la Figura 3.2.7.

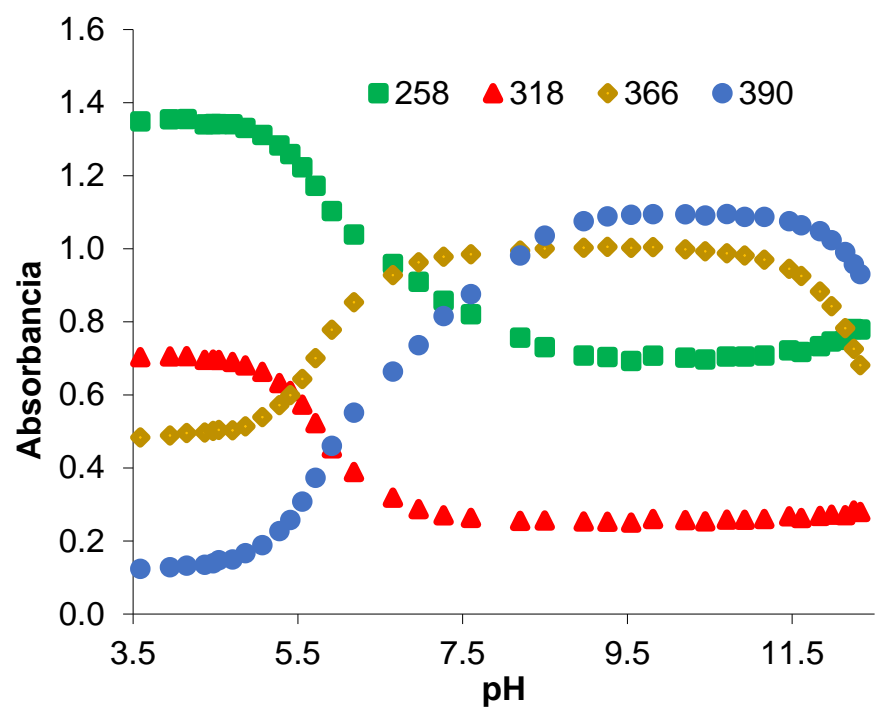

Figura 3.2.8. Gráficas de absorbancia en función del $\mathrm{pH}$, a partir de la variación del $\mathrm{pH}$ de mangiferina $4 \times 10^{-5} \mathrm{M}$ en presencia de $\beta C D 10 \mathrm{mM}$, para las $\lambda_{\text {máx }}=258(\bullet), 318(\mathbf{\Delta}), 366(\diamond), 390(\bullet)$ $n m$.

En cada curva de la Figura 3.2.8 se pueden observar cambios de pendiente que se deben a diferentes valores de pK'a para cada equilibrio generalizado de acidez. 


\subsubsection{Determinación de constantes de acidez condicionales de la mangiferina con el programa $S Q U A D$ para $[\beta C D]=10 \mathrm{mM}$}

Experimentalmente se obtuvieron 45 espectros de absorción por cada valor de $\mathrm{pH}$ corregido con la ecuación 3.1 y medidos dentro del intervalo de longitudes de onda de 240 - $500 \mathrm{~nm}$ con incrementos de $2 \mathrm{~nm}$, con esta información se alimenta el programa SQUAD. Utilizando el mismo modelo de cuatro equilibrios generalizados, que en el caso anterior.

Los resultados de los valores de las constantes condicionales de formación global, obtenidos por SQUAD, se muestran en la Tabla 3.2.

Tabla 3.2. Constantes condicionales de formación global de mangiferina en presencia de $\beta$-ciclodextrina $10 \mathrm{mM}$, obtenidas con el programa SQUAD.

\begin{tabular}{cc}
\hline $\begin{array}{c}\text { Equilibrio generalizado de } \\
\text { formación global }\end{array}$ & $\log \beta_{i}^{\prime} \pm \sigma_{\text {const }}$ \\
\hline$M G F^{\prime}+H^{+}=H M G F^{\prime}$ & $\log \beta^{\prime}{ }_{1}=12.714 \pm 0.005$ \\
$M G F^{\prime}+2 H^{+}=H_{2} M G F^{\prime}$ & $\log \beta_{2}^{\prime}{ }_{2}=23.583 \pm 0.040$ \\
$M G F^{\prime}+3 H^{+}=H_{3} M G F^{\prime}$ & $\log \beta^{\prime}{ }_{3}=31.299 \pm 0.041$ \\
$M G F^{\prime}+4 H^{+}=H_{4} M G F^{\prime}$ & $\log \beta^{\prime}{ }_{4}=35.156 \pm 0.042$ \\
\hline $\begin{array}{l}\sigma_{\text {const }}=\text { desviación estándar sobre la constante; } \\
\sigma_{\text {tot }}=\text { desviación estándar total sobre la regresión }=6.38 \times 10^{-3}\end{array}$
\end{tabular}

Con SQUAD también es posible obtener los coeficientes de absortividad molar, como ya fue mostrado anteriormente, para cada especie generalizada presente en el sistema; así como los cambios que tuvo al aumentar la concentración de $\beta$-CD. Así, en la Figura 3.2.9 se muestran los coeficientes de absortividad molar con sus respectivas desviaciones estándar para las 5 especies generalizadas de mangiferina, con $\beta$-ciclodextrina $10 \mathrm{mM}$. Se puede ver, por ejemplo, que para la 
especie MGF' se observan 5 máximos representativos en 242, 254, 282, 344 y 418 nm. En el caso de las especies HMGF', $\mathrm{H}_{2} \mathrm{MGF}^{\prime}, \mathrm{H}_{3} \mathrm{MGF}^{\prime}$ y $\mathrm{H}_{4} \mathrm{MGF}$ 'los cambios son menos importantes.

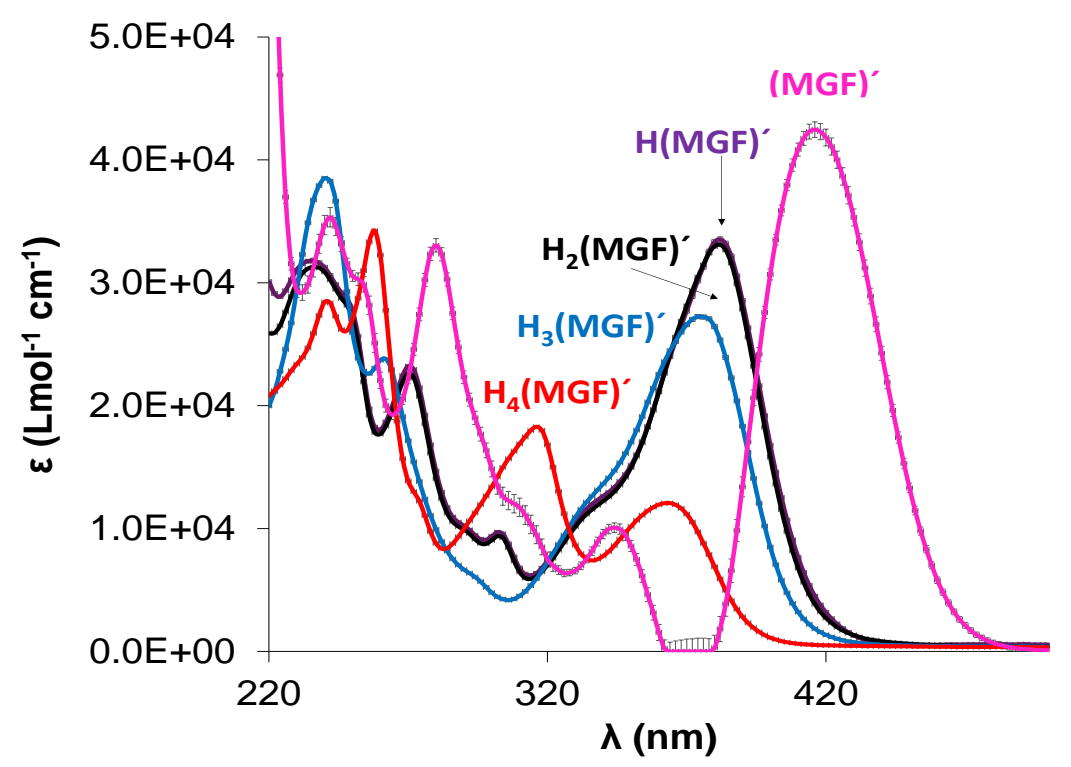

Figura 3.2.9. Coeficientes de absortividad molar de las diferentes especies generalizadas de mangiferina en presencia de $\beta C D$ a $10 \mathrm{mM}$, obtenidos por SQUAD en el intervalo de $3.586 \leq p H \leq 12.326$.

\subsubsection{Comparación de los resultados para la mangiferina en ausencia de $\beta C D$ y para $[\beta C D]=10 \mathrm{mM}$}

Por medio del uso de una hoja de cálculo de Excel se construye el modelo teórico como se describió anteriormente, ahora tomando los nuevos valores de las constantes condicionales de formación para una concentración de $\beta C D=10 \mathrm{mM}$, para poder ajustar los datos experimentales con los resultados obtenidos por SQUAD. 
En la Figura 3.2.10 se muestran las gráficas de absorbancia en función del pH a las diferentes longitudes de onda representativas para el conjunto de espectros de absorción de la Figura 3.2.7. y su ajuste representado con la línea continua.
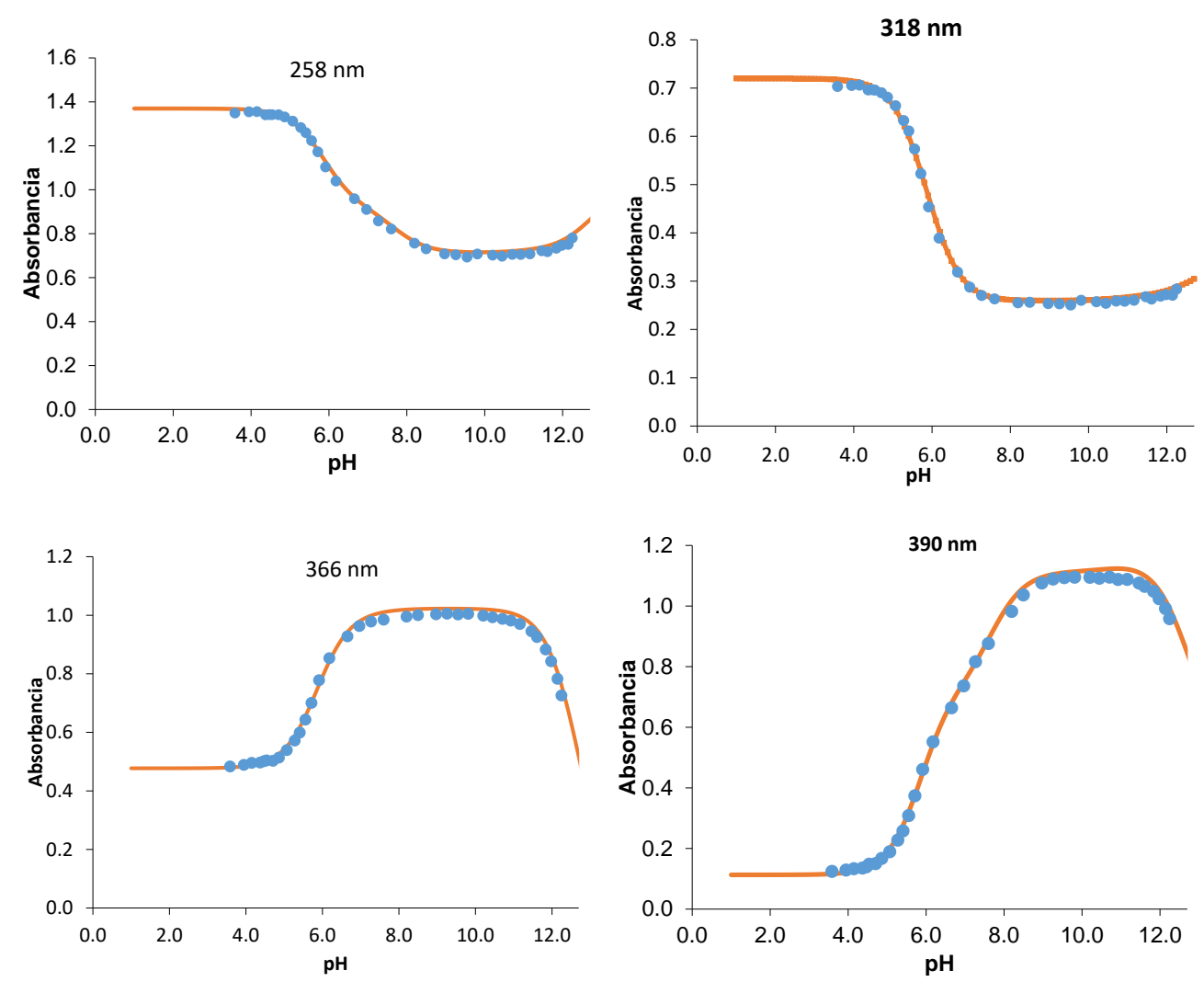

Figura 3.2.10. Ajuste de las curvas de absorbancia en función del valor de $\mathrm{pH}$ representado para diferentes longitudes de onda (258nm, 318nm 366nm y 390nm), donde los marcadores representan los datos experimentales y la línea continua indica el ajuste.

El ajuste de estas curvas es todavía mejor que el observado en la Figura 3.2.4 (a $[\beta C D]=1 \mathrm{mM})$. Esto confirma que el modelo propuesto en SQUAD, de 5 especies y 4 valores de log $\beta_{i}^{\prime}$ sigue la misma tendencia que marcan las curvas experimentales.

Una vez que se tiene el refinamiento para el valor de las contantes condicionales de formación global, con el programa SQUAD y mediante el uso de la ley de Hess, se pueden obtener los valores de las constantes condicionales de acidez ( $\mathrm{pK}^{\prime}{ }_{\mathrm{ai}}$ ) para 
cada equilibrio de las especies $\mathrm{H}_{4} \mathrm{MGF}^{\prime}, \mathrm{H}_{3} \mathrm{MGF}^{\prime}, \mathrm{H}_{2} \mathrm{MGF}^{\prime}, \mathrm{HMGF}^{\prime}, \mathrm{MGF}^{\prime}$ con la concentración de $\beta C D$ impuesta $10 \mathrm{mM}$. Dichos resultados se muestran en la Tabla 3.4 y el diagrama de distribución de las especies generalizadas de la mangiferina en función del pH se muestra en la Figura 3.2.11.

Tabla 3.4. Valores de pKa de especies de mangiferina y de pKa' de especies generalizadas de mangiferina en presencia de $\beta$-ciclodextrina $10 \mathrm{mM}$.

\begin{tabular}{ccc}
\hline $\begin{array}{c}\text { Equilibrio generalizado de } \\
\text { acidez }\end{array}$ & Valor reportado ${ }^{12}$ & Experimental \\
\hline$H_{4} M G F^{\prime}=H_{3} M G F^{\prime}+H^{+}$ & $\mathrm{pK}_{\mathrm{a} 1=6.52 \pm 0.06}$ & $\mathrm{pK}_{\mathrm{a} 1}^{\prime}=5.857 \pm 0.059$ \\
$H_{3} M G F^{\prime}=H_{2} M G F^{\prime}+H^{+}$ & $\mathrm{pK}_{\mathrm{a} 2=7.97 \pm 0.06}$ & $\mathrm{pK}_{\mathrm{a} 2}^{\prime}=7.716 \pm 0.057$ \\
$H_{2} M G F^{\prime}=H M G F^{\prime}+H^{+}$ & $\mathrm{pK}_{\mathrm{a} 3=9.44 \pm 0.04}$ & $\mathrm{pK}_{\mathrm{a} 3=10.869 \pm 0.040}^{\prime}$ \\
$H M G F^{\prime}=M G F^{\prime}+H^{+}$ & $\mathrm{pK}_{\mathrm{a} 4=12.10 \pm 0.01}$ & $\mathrm{pK}_{\mathrm{a} 4=12.714 \pm 0.005}$ \\
\hline
\end{tabular}

En la Figura 3.2.11 se muestra el diagrama de distribución de las especies generalizadas de mangiferina con $[\beta C D]=10 \mathrm{mM}$. En dicha figura se representan con marcadores las fracciones de las especies generalizadas, en tanto que en línea continua se representan las fracciones de las especies de mangiferina en ausencia de $\beta C D$, reportados en la literatura ${ }^{12}$.

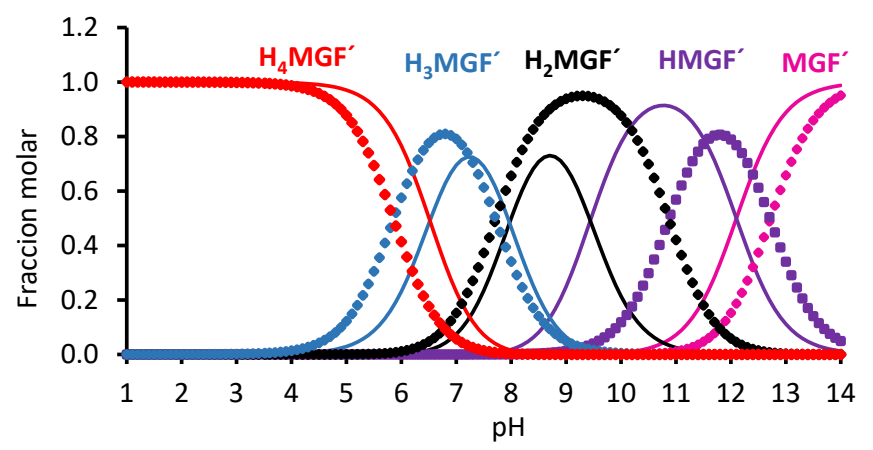

Figura 3.2.11. Diagrama de distribución de especies, para sistemas de mangiferina.

En línea continua se representan las especies correspondientes al sistema de la mangiferina, en ausencia de $\beta C D$, en tanto que las fracciones de las especies generalizadas de mangiferina en presencia de la $\beta C D$ a $10 \mathrm{mM}$, se representan con marcadores. 
Los valores de $\mathrm{pK}^{\prime}{ }_{\mathrm{a} 1}$ y $\mathrm{pK}^{\prime}{ }_{\mathrm{a} 2}$ mostraron una disminución con respecto a $\mathrm{pK}_{\mathrm{a} 1}$ y $\mathrm{pK}_{\mathrm{a} 2}$, en el caso de los valores de $\mathrm{pK}^{\prime}{ }_{\text {a3 }}$ y $\mathrm{pK}^{\prime}{ }_{44}$ presentaron un aumento en comparación con los valores reportados en la literatura. Esto ocasiona que la especie $\mathrm{H}_{2} \mathrm{MGF}^{\prime}$ aumente significativamente su fracción molar (con respecto a lo que se observa en ausencia de $\beta C D$ ) y el intervalo de predominio de $\mathrm{pH}$ esta especie.

En la Figura 3.2.11. se puede visualizar que la concentración presente de $\beta C D$, tiene un efecto en el cambio de las constantes de acidez de la mangiferina, con respecto a lo observado en la Figura 3.2.5. Debido a esto es posible afirmar que las constantes condicionales de acidez de las especies de la mangiferina dependen de la concentración de $\beta C D$, con lo que se pone en evidencia que, prácticamente, todas las especies de la mangiferina forman complejos de inclusión con la $\beta C D$ y que, al parecer, los más estables son los que corresponden a los anfolitos $\left(\mathrm{H}_{3} \mathrm{MGF}^{-}\right.$,

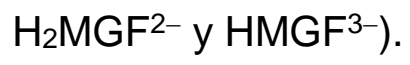

Finalmente, en la Figura 3.2.12, se comparan los coeficientes de absortividad molar de las especies de mangiferina en ausencia de $\beta C D$ (línea continua) y los de las especies generalizadas de mangiferina con $\beta C D 10 \mathrm{mM}$ (marcadores).

Las diferencias que se presentan en los desplazamientos hacia longitudes de onda menores de las especies mangiferina con $\beta C D$ son mayores en la Figura 3.2.12 con respecto a los observados en la Figura 3.2.6. Esto se puede interpretar como un aumento en las interacciones que hay entre las especies de la mangiferina con $\beta C D$ conforme aumenta su concentración.

Como se ha demostrado que al aumentar la concentración de $\beta C D$ se ven mayores cambios en los valores de pK'a de la diferentes especies generalizadas de la mangiferina, la inclusión de las especies de mangiferina en la cavidad de la $\beta C D$ debe hacerse por el grupo xantona. Es así que, en la Figura 3.2.12, se presenta una posible estructura química del complejo de inclusión para la especie $\mathrm{H}_{4} \mathrm{MGF}(\beta C D)$. $\mathrm{Si}$, por el contrario, el grupo glucosídico se incluyera en la cavidad de la $\beta C D$; se esperaría observar prácticamente los mismos valores de constantes de acidez para la mangiferina en presencia de $\beta C D$. 

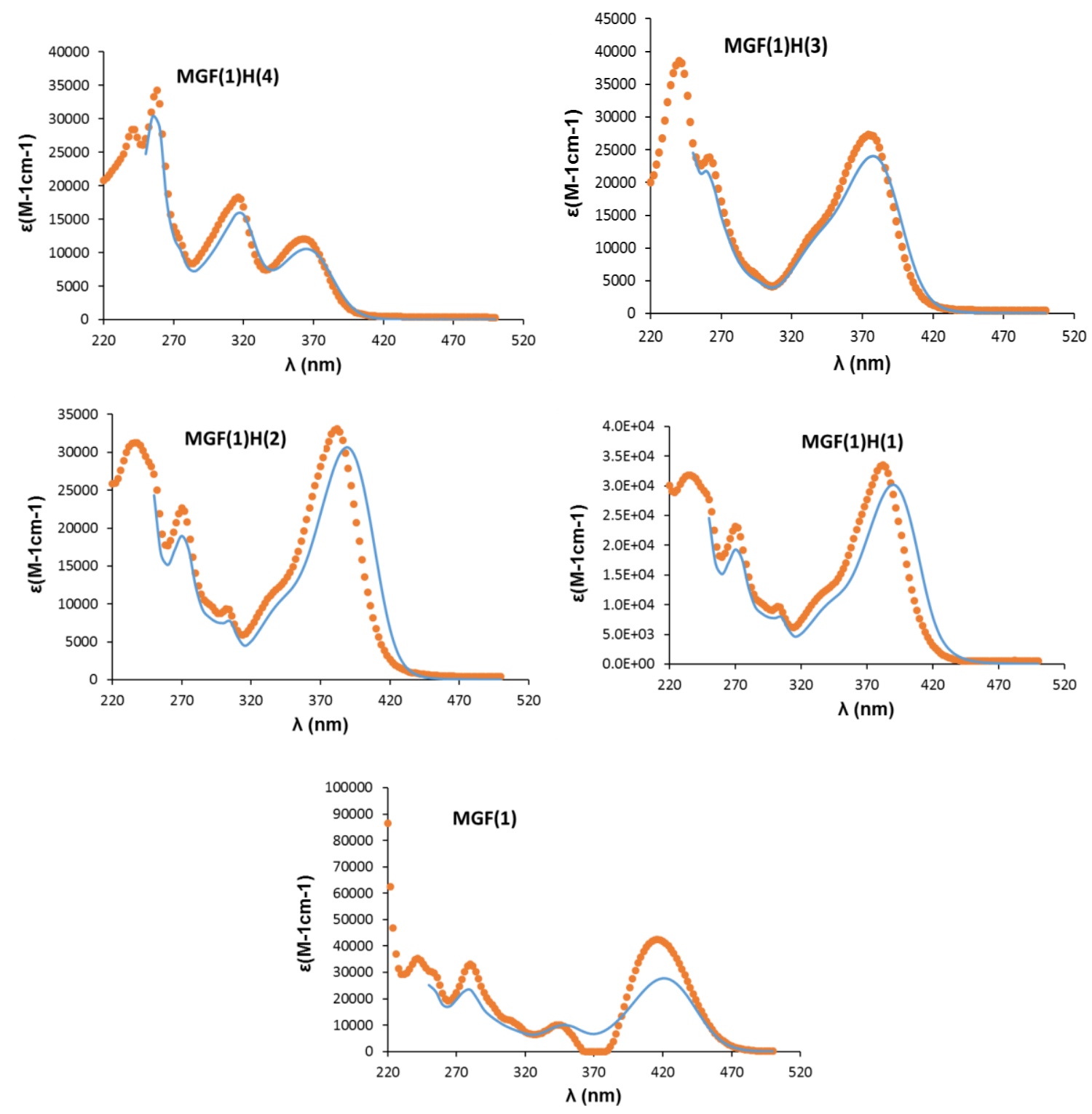

Figura 3.2.12. Coeficientes de absortividad molar para cada especie generalizada de mangiferina. Con línea continua se representan los coeficientes de las especies en ausencia de $\beta-C D$, y con marcadores se representan los coeficientes de las especies generalizadas cuando se ha impuesto $[\beta-C D]=10 \mathrm{M}$. 


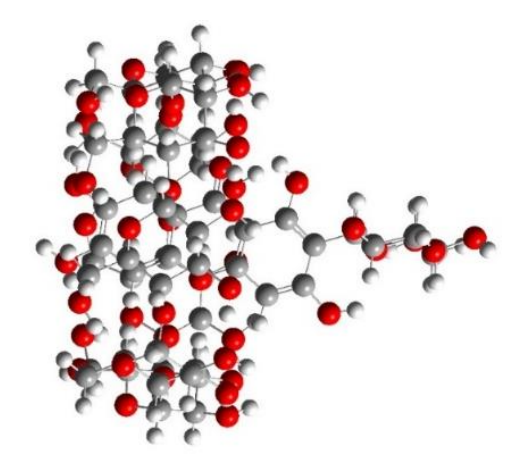

Figura 3.2.13. Posible estructura química del complejo de inclusión, propuesta debida a los valores de las constantes condicionales de acidez para la especie $H_{4} M G F(\beta C D)$, que se lleva a cabo por el grupo xantona hacia el interior de la $\beta C D$, y por el lado más amplio.

\subsection{Conclusiones}

Se determinaron los cuatro valores de pKa' de la mangiferina a dos concentraciones impuestas de $\beta$-ciclodextrina en el sistema ( $1 \mathrm{mM}$ y $10 \mathrm{mM}$ ), en medio acuoso y por Espectroscopía UV-visible. Estos valores se compararon con los valores reportados en la literatura ${ }^{12}$ para la mangiferina.

Se encontró que el comportamiento el efecto de la $\beta$-ciclodextrina sobre la acidez de las especies de la mangiferina es tal que se estabilizan los anfolitos del sistema, sobre todo el $\mathrm{H}_{2} \mathrm{MGF}^{2-}$.

Debido a que los cambios en los valores de las constantes condicionales de acidez de las especies de la mangiferina es mayor al aumentar la concentración de la $\beta C D$, es posible afirmar que la formación de los complejos se lleva a cabo por la inclusión de la xantona hacia ciclodextrina y no por el grupo glucosídico. 


\section{CAPÍTULO 4}

\section{DETERMINACIÓN DE LA CONSTANTE DE INCLUSIÓN DEL COMPLEJO MANGIFERINA/ $\beta$ - CICLODEXTRINA POR EL MÉTODO DE HIGUCHI- CONNORS}




\section{CAPÍTULO 4.}

\section{DETERMINACIÓN DE LA CONSTANTE DE INCLUSIÓN DEL COMPLEJO MANGIFERINA/ $\beta$-CICLODEXTRINA POR EL MÉTODO DE HIGUCHI-CONNORS}

Este capítulo explica la determinación de la constante de inclusión por el método de diagrama de fases propuesto por Higuchi y Connors, ${ }^{42},{ }^{43}$, con el fin de evidenciar que la $\beta-C D$ mejora la solubilidad de la Mangiferina en solución acuosa. Como se indicó en el Capítulo 1, la baja solubilidad es un problema si se quiere administrar la mangiferina como complemento alimenticio o como fármaco por vía oral.

\subsection{Preparación de Soluciones}

Se llevó a cabo la preparación de diferentes soluciones de $\beta C D$ (Sigma-Aldrich, $95 \%$ ), entre $0-0.01633 \mathrm{M}$ en $10 \mathrm{~mL}$ de agua desionizada; posteriormente a cada solución se le adicionó una cantidad de $\mathrm{H}_{4} \mathrm{MGF}$, pesada con exactitud y precisión, que asegurara soluciones saturadas de mangiferina, sabiendo que el valor reportado de solubilidad de mangiferina en agua es de $0.111 \mathrm{mg} / \mathrm{mL}^{44,45}$.

\subsubsection{Procedimiento experimental}

Las soluciones preparadas se colocan en agitación constante por 24 horas a temperatura ambiente, pasado este intervalo de tiempo las soluciones se filtran usando una membrana de nylon de $0.45 \mu \mathrm{m}$, Merck Millipore. A continuación los filtros tarados, con el residuo, son secados en estufa y pesados para obtener la cantidad disuelta de mangiferina por diferencia de pesos. Finalmente cada solución es medida por Polarimetría, espectrofotometría UV-visible y el valor de $\mathrm{pH}$. 
Los valores de $\mathrm{pH}$ de las soluciones se midieron con un potenciómetro $\mathrm{HACH}$ equipado con un electrodo de $\mathrm{Ag} / \mathrm{AgCl}$, dando en todos los casos un valor de $\mathrm{pH}$ dentro del intervalo de $4.6 \leq \mathrm{pH} \leq 5.4$.

En Polarimetría se utilizó un polarímetro MCP 350 de la marca Anton Paar, equipado con una celda de $100 \mathrm{~mm}$ con control de temperatura a $(25.0 \pm 0.5){ }^{\circ} \mathrm{C}$, mediante un sistema Peltier, y una lámpara de tungsteno con dos filtros longitud de onda, de 325 y $589 \mathrm{~nm}$. En este trabajo se determinó el ángulo de rotación, o rotación óptica, a la longitud de onda de la línea $D$ del sodio ( $589 \mathrm{~nm}$ ).

Los espectros de absorción se midieron con un equipo Perkin Elmer Lambda 950, equipado con una celda de cuarzo con paso óptico de $1 \mathrm{~cm}$, de $250-450 \mathrm{~nm}$.

\subsection{Resultados y discusión}

Muchos estudios indican que las ciclodextrinas pueden mejorar la solubilidad de sustancias orgánicas poco solubles en agua ${ }^{46,47}$ al formarse complejos de inclusión 48,49,50,51,52,53,54. En estos trabajos utilizan el método propuesto por HiguchiConnors ${ }^{42,43}$, descrito en el apéndice $\mathrm{G}$, para determinar la constante de inclusión de un complejo formado, basado en un diagrama de solubilidad-fases, $\left(\left[G_{0}\right]=f([\beta C D])\right.$ de soluciones saturadas y usando la ecuación 4.1, que es para la formación de complejos tipo $G(C D)$.

$$
K_{1: 1}=\frac{\alpha}{G_{0}(1-\alpha)}
$$

donde $[G]$ representa la solubilidad del huésped en ausencia de $\beta C D$ y $\alpha$ indica la pendiente de la recta obtenida en el diagrama de solubilidad, en el caso que dicho diagrama tenga tendencia lineal.

En este capítulo el huésped es la especie $\mathrm{H}_{4} \mathrm{MGF}$, porque en el intervalo de $\mathrm{pH}$ trabajado entre $4.6 \leq \mathrm{pH} \leq 5.4$, es la especies predominante. 
En la Tabla 4.1, se presentan los resultados de la solubilidad de mangiferina en presencia de $\beta-C D$.

Tabla 4.1. Resultados experimentales de solubilidad de mangiferina en presencia de $\beta C D$.

\begin{tabular}{|c|c|c|c|c|c|}
\hline Soluciones & $\begin{array}{c}\mathrm{m}\left(\mathrm{H}_{4} \mathrm{MGF}\right) / \mathrm{g} \\
\text { (inicial) }\end{array}$ & $\begin{array}{c}\mathrm{m}\left(\mathrm{H}_{4} \mathrm{MGF}\right) / \mathrm{g} \\
\text { (soluble) }\end{array}$ & $\begin{array}{c}\mathrm{m}\left(\mathrm{H}_{4} \mathrm{MGF}\right) / \mathrm{g} \\
\text { (insoluble) }\end{array}$ & $\begin{array}{c}{\left[\mathrm{H}_{4} \mathrm{MGF}\right] / \mathrm{M}} \\
\text { (soluble) }\end{array}$ & {$[\beta C D] / M$} \\
\hline 1 & 0.00340 & 0.00126 & 0.00214 & 0.000298 & 0 \\
\hline 2 & 0.00462 & 0.00186 & 0.00276 & 0.000440 & 0.0019 \\
\hline 3 & 0.00680 & 0.00329 & 0.00351 & 0.000779 & 0.0054 \\
\hline 4 & 0.00868 & 0.00447 & 0.00421 & 0.001058 & 0.0091 \\
\hline 5 & 0.00980 & 0.00533 & 0.00447 & 0.001262 & 0.0110 \\
\hline 6 & 0.01311 & 0.00790 & 0.00521 & 0.001871 & 0.0145 \\
\hline
\end{tabular}

Con la información de la Tabla 4.1 se construye el diagrama de solubilidad-fases, presentado en la Figura 4.1.

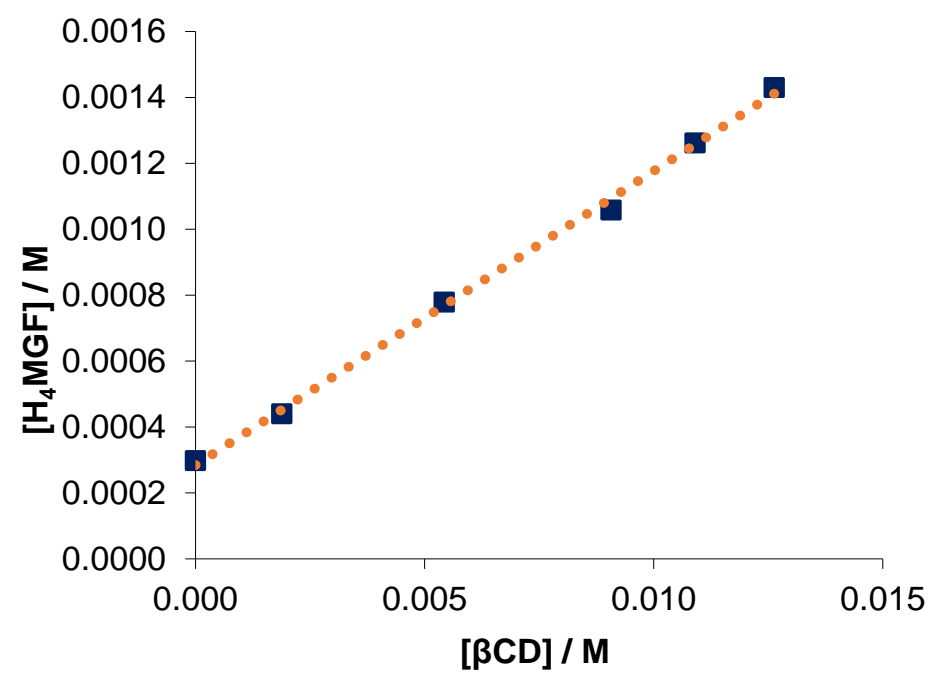

Figura 4.2.1 Diagrama de solubilidad-fases de $\mathrm{H}_{4} M G F^{\prime}$ con la presencia de diferentes concentraciones de $\beta-C D$ dentro de un intervalo de $0-0.01 \mathrm{M}$. 
La gráfica de la Figura 4.2.1 presenta una tendencia claramente lineal; representada con la ecuación 4.2:

$$
\left[H_{4} M G F\right]=0.089221[\beta \mathrm{CD}]+0.000285
$$

con un coeficiente de correlación de 0.997989. Es por ello que se puede considerar como un diagrama de tipo $A$, de acuerdo a la clasificación de Higuchi y Connors ${ }^{42}$; lo que implica la formación de complejos de inclusión que aumentan la solubilidad de la mangiferina con posibles estequiometrias $\mathrm{H}_{4} \mathrm{MGF}(\beta \mathrm{CD}),\left(\mathrm{H}_{4} \mathrm{MGF}\right)_{2}(\beta C D), \ldots$, $\left(\mathrm{H}_{4} \mathrm{MGF}\right) \mathrm{m}(\beta \mathrm{CD})$.

En este caso se supone la formación del complejo $\mathrm{H}_{4} \mathrm{MGF}(\beta C D)$, debido a que, como se mostró en el capítulo 2 a pH 8.6 el complejo $\mathrm{H}_{2} \mathrm{MGF}(\beta C D)$, aproximadamente, es el que mejor explica los resultados espectrofotométricamente medidos.

Con esta información se puede determinar el valor de la constante de inclusión para el complejo $\mathrm{H}_{4} M G F(\beta C D)$, sustituyendo la pendiente y ordenada al origen de la ecuación 4.2 en la ecuación 4.1, muestra.

$$
K_{H_{4} \operatorname{MGF}(\beta C D)}=\frac{\alpha}{\left[H_{4} M G F\right]_{0}(1-\alpha)}=\frac{0.089221}{(0.000285)(1-0.089221)}=343.723
$$

La $\beta C D$ es una molécula que por Espectroscopía UV-visible no puede ser cuantificada ya que no presenta absorción en la región visible y muy poca absorción en la región ultravioleta (cercana a los $200 \mathrm{~nm}$ ), pero sí cuenta con la propiedad de ser ópticamente activa y la mangiferina podría serlo también debido a su grupo glucosídico sustituido (aunque su capacidad para rotar el plano de la luz polarizada plana es pequeña). Por lo tanto, es posible medir la rotación óptica de las soluciones preparadas, donde los resultados se muestran en la Figura 4.2.2. 


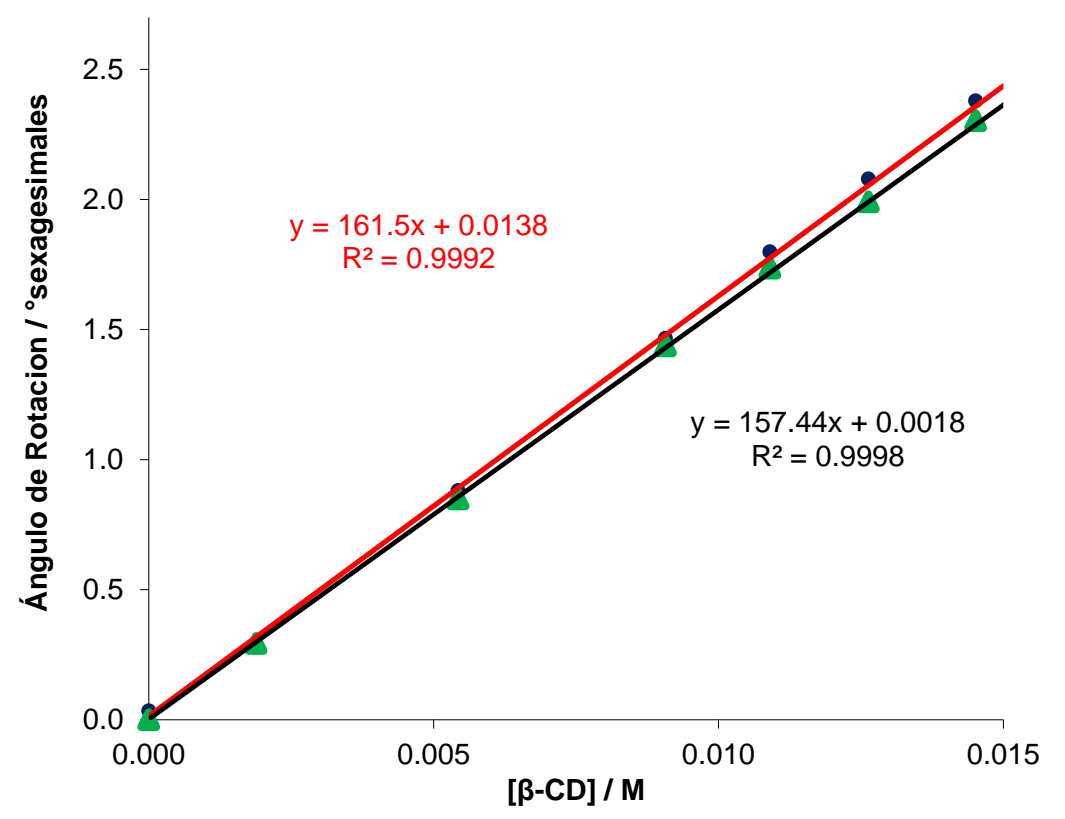

Figura 4.2.2 Ángulo de rotación en función de la concentración de $\beta-C D$, a 589 nm. En negro se muestra la curva de $\beta C D$ sin mangiferina y en rojo la curva de las soluciones saturadas de mangiferina con $\beta C D$.

La Figura 4.2.2 muestra el efecto de mangiferina incluida en la $\beta C D$ sobre la rotación óptica de esta última; aunque, como se puede ver, este efecto es pequeño. Sin embargo, confirma la interacción entre las especies $\beta C D$ y $\mathrm{H}_{4} M G F(\beta C D)$.

La mangiferina es una sustancia que se puede seguir espectrofotométricamente, y mostrar el efecto que tiene sobre ella la $\beta C D$. Por esto se midieron los espectros de absorción presentan en la Figura 4.2.3. 


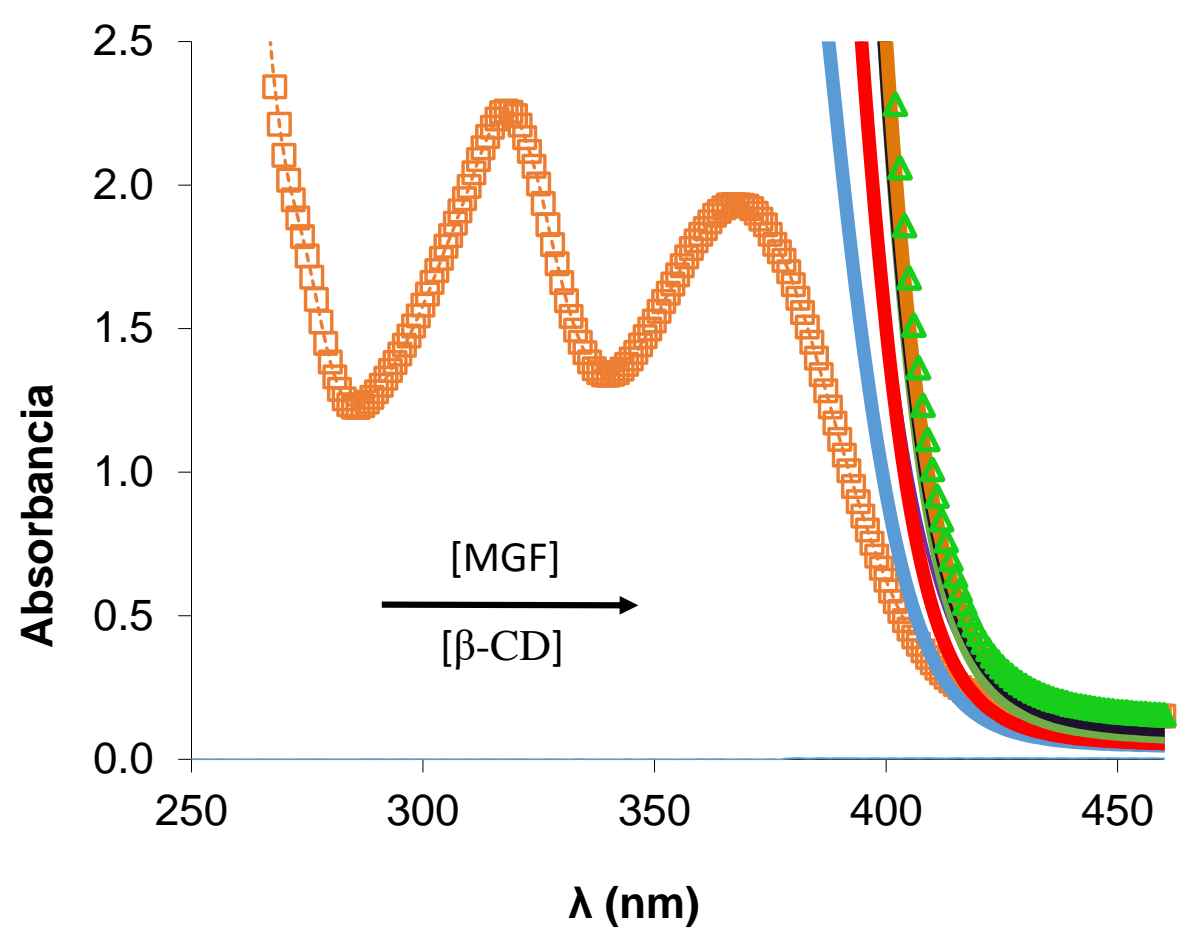

Figura 4.2.3. Conjunto de espectros de absorción de mangiferina dentro de un intervalo de concentración en (0.000298 - 0.002197) $M y$ de $\beta-C D$ en (0 - 0.01633) $M$, de forma creciente cada concentración. Los espectros marcados por la línea punteada representan el inicio y el final del experimento.

La Figura 4.2.3 se muestra el efecto que tiene la $\beta C D$ sobre la mangiferina, al aumentar la absorbancia en cada solución llegando a la saturación del detector en longitudes de onda menores a $390 \mathrm{~nm}$. Esto confirma el aumento de la solubilidad de la mangiferina al aumentar la concentración de $\beta C D$ y lo que indica la formación de complejos de inclusión. 


\subsection{Conclusiones}

En este capítulo, se puso en evidencia la formación de complejos de inclusión debido al aumento de la solubilidad de la mangiferina con la concentración de $\beta C D$. Con el estudio del diagrama de solubilidad-fases se logró determinar la constante de inclusión $K_{H_{4} \operatorname{MGF}(\beta C D)}=343.723$ que es equivalente a decir $\log K_{H_{4} \operatorname{MGF}(\beta C D)}=2.54$.

Para demostrar que el aumento de la solubilidad de mangiferina se afectado por la $\beta C D$ fue posible medir los espectros de absorción que confirman dicha hipótesis.

Las mediciones de polarimetría mostraron que la mangiferina aumenta el ángulo de rotación de la $\beta C D$, aunque la diferencia fue más apreciable en valores altos de concentración de Mangiferina. 


\section{Conclusiones generales y perspectivas}

Se puso en evidencia que existe la formación de complejos de inclusión, no solo para la especie $\mathrm{H}_{4}$ MGF sino de todas las demás especies en sus diferentes grados de desprotonación. Utilizando diferentes técnicas experimentales, tales como: espectrofotometría UV-visible, fluorescencia, polarimetría y gravimetría (por diagrama de solubilidad-fases).

Al parecer la estequiometría más probable para los complejos de inclusión es de 1 mangiferina con $1 \beta C D$, ya que los modelos utilizados con esta estequiometría explican bien los resultados obtenidos a pH de 5 como a pH de 8.6.

Los estudios en función del pH justifica la formación de complejos de inclusión para todas las especies de mangiferina, principalmente para la especie $\mathrm{H}_{2} \mathrm{MGF}^{2-}$, que presenta un aumento en su fracción molar al estar acomplejada con un valor de

$\log K_{H_{2} \operatorname{MGF}(\beta C D)^{2-}}=3.4$, y la especie $\mathrm{H}_{4}$ MGF que al $\log K_{H_{4} \operatorname{MGF}(\beta C D)}=2.54$, por la diferencia en estos valores se puede decir que el complejo más estable es $\mathrm{H}_{2} \mathrm{MGF}(\beta C D)^{2-}$.

Los estudios presentados en los capítulos anteriores se refuerzan por que no entran en contradicción, lo que hace que los modelos propuestos sean verosímiles.

Como perspectivas:

* Realizar estudios a diferentes valores de pH's donde existan especies predominantes según el diagrama de distribución de especies de mangiferina para la formación de complejos de inclusión, y en caso de existir complejos determinar sus constantes de inclusión junto con su estequiometría, utilizando el método robusto.

* Estudiar la solubilidad de los complejos de inclusión mediante la influencia de diferentes medios de $\mathrm{pH}$, además de poder estudiar a 
diferentes temperaturas, encontrando así las condiciones más favorables para obtener una mayor solubilidad de este complejo.

* Estudiar complejos de inclusión con derivados de CD’s y MGF así como en presencia de iones metálicos.

* Finalmente, estudiar por métodos teóricos las diferentes posibles estructuras químicas o formas de complejación entre mangiferina y $\beta C D$, que ayuden a confirmar la estructura propuesta en este trabajo. 


\section{Referencias}

${ }^{1}$ Kumar P., Kapur S. Mangiferin: A potential natural molecule for management of metabolic syndrome. Int J Pharm. (2015); 7, 9-13.

2 Lipinski Ch. A. Lead- and drug-like compounds: the rule-of-five revolution. Drug Discovery Today: Technologies. (2004); 1(4), 337-341.

${ }^{3}$ Lipinski Ch. A., Lombardo F., Dominy B., Feeney P. Experimental and computational approaches to estimate solubility and permeability in drug discovery and development settings. Advanced Drug Delivery Reviews. (2001); 46, 3-26.

${ }^{4}$ Vyas, A.; Syeda, K.; Ahmad, A.; Padhye, S.; Sarkar, F. H., Perspectives on Medicinal properties of Mangiferin. Med. Chem. (2012); 12(5), 412-425.

5 Outhiriaradjou B., Yuling Ch. Medicinal Properties of Mangiferin, Structural Features, Derivative Synthesis, Pharmacokinetics and Biological Activities. Med Chem. (2015); 15(7), 582-594.

6 Miura T., Ichiki H., Hashimoto I., Iwamoto N. Antidiabetic activity of xanthone compounds, mangiferin. Phytomedicine. (2001); 8(2), 85-87.

7 Sánchez G. M, Re L. Giuliani A. Núñez-Sellés A. J. Protective effects of mangifera indica L. extract, mangiferina and selected antioxidants against TPA-induced activation in mice. Pharmacological Reseach. (2000); 42(6), 565-573.

${ }^{8}$ Garrido G, González D, Delporte C, Backhouse N, Quintero G, Núñez-Sellés AJ. Analgesic and anti-inflammatory effects of Mangifera indica L. extract (Vimang). Phytother Res. (2001); 15, 18-21.

${ }_{9}^{9}$ Matkowski A, Kuś P, Góralska E, Woźniak D. Mangiferin-a bioactive xanthonoid, not only from mango and not just antioxidant. Med Chem. (2013); 13, 439-55.

10 Ghosal S., Rao. A plausible chemical mechanism of the bioactivities of mangiferin. Indian. Journal of Chemistry. (1996); 35B, 561-566.

${ }^{11}$ Raihan HM, Nan C, Yuling C. Therapeutic potential of the natural product mangiferin in metabolic syndrome. J Nutr Ther. (2013); 2, 74-9.

12 Gomez-Zaleta, B.; Ramirez-Silva, M. T.; Gutierrez, A.; Gonzalez-Vergara, E.; Guizado Rodriguez, M.; Rojas-Hernandez, A., UV/vis, 1H, and 13C NMR Spectroscopic studies to determine mangiferin pKa values. Spectrochimica Acta Part A: Molecular and Biomolecular Spectroscopy. (2006); 64(4), 1002-1009.

13 Mendoza-Sarmiento G.; Rojas-Hernández A.; Galano A. and Gutiérrez A., A combined experimental-theoretical study of the acid-base behavior of mangiferin: implications for its antioxidan activity. RCS Adv. (2016); 6, 51171-51182.

${ }^{14} \mathrm{Ma} \mathrm{H}$, Chen $\mathrm{H}$, Sun L, Tong L, Zhang T. La mejora de la permeabilidad y la absorción oral de manguiferina por complejación fosfolípido. Fitoterapia. (2014); 93, 54-61.

${ }^{15}$ Kaur R., Ranjot Kaur , Kaur M., Rajpreet Kaur , Jasleen Kaur , Harpreet Kaur , 4 y Bhupinder Singh. Exploración y validación de las propiedades fisicoquímicas de manguiferina través GastroPlus ${ }^{\circledR}$ software. Future Sci. OA (2017); 3(1), 5623-5635. 
16 De Souza JRR, Feitosa. EMP el secado por pulverización encapsulación de mangiferina usando polímeros naturales. Hydrocoll alimentos. (2013); 33(1), 10-18.

17 Moura U, Barbosa M, Genro C, Seibel D, Gomes P, Raffin P. Desarrollo y caracterización de nanocápsulas mangiferina. J. Pharm. Pharmacogn. Res. (2014); 2(1), 34.

${ }^{18}$ Zheng-Yu Jin. Cyclodextrin Chemical: Preparation and Aplication. World Scientific: USA, (2007); 3-16.

19 E.M. Martin Del Valle. Cyclodextrins and their uses: A review. Process Biochemistry, (2004); 39,1033-1046.

20 Cserhati T., Forgacs E. Cyclodextrins in Chromatography. RS.C Chromatography Monographs; Cambridge, UK. (2003); 1-10.

21 Szejtli J. Introduction and General Overview of Cyclodextrin Chemistry. Chem. Rev. (1998); 98, 1743-1753.

22 Hernández-Benito J., González-Mancebo S., Calle E., García-Santos M., Casado J. A Practical Integrated Approach to Supramolecular Chemistry. I. Equilibria in Inclusion Phenomena. J. Chem. Ed. (1999); 76 (3), 419-421.

${ }^{23}$ Uekama K.; Hirayama F.; Irie T. Cyclodextrin Drug Carrier Systems. Chem. Rev. (1998); 98, 20452076.

24 V.M. Rekharsky, Y. Inoue. Complexation Thermodynamics of Cyclodextrins Chem. Rev. (1998); 98, 1875-1917.

25 Tingry S., Innocent C., Touil S., Deratani A. Carbon paste biosensor for phenol detection of imprégnate tissue: modification of selectivity by using $\beta$-cyclodextrin-containing PVA membrame. Materials Science and Engineering: C. (2006); 26, 222-226.

${ }^{26}$ Hedges A. Industrial Applications of Cyclodextrins. Chem. Rev. (1998); 98, 2035-2044.

${ }^{27}$ Folch-Cano C., Jullian C., Speisky H., C. Olea-Azar. Antioxidant activity of inclusion complexes of tea catechins with $\beta$-cyclodextrins by ORAC assays. Food Research International. (2010); 43, 20392044.

${ }^{28}$ Wen Chen, Li-Juan Yang, Shui-Xian M, Xiao-Dong Yang, Bao-Min Fan, Jun Lin. Crassicauline A/ $\beta$ cyclodextrin host-guest system: Preparation, characterization, inclusion mode, solubilization and stability. Carbohydrate Polymers. (2011); 84, 1321-1328.

${ }^{29}$ Sau S., Solanki B., Orprecio R., Stam J. V., Evans Ch. H. Higher-Order Cyclodextrin Complexes: The Naphthalene System. Journal of Inclusion Phenomena and Macrocyclic Chemistry. (2004); 48, 173-180.

${ }^{30}$ Astray G.; Gonzalez-Barriento C.; Mejuto J.C.; Rial-Otero R.; J. Simal-Gándara. A review on the use of cyclodextrins in foods. Food Hydrocolloids. (2009); 23, 1631-1640.

33 Rekharsky V. Mikhal, Yoshihica Inoue. Complexation Thermodynamics of Cyclodextrins. Chem. Rev. (1998); 98, 1875-1917.

${ }^{31}$ Rekharsky V. Mikhal, Yoshihica Inoue. Complexation Thermodynamics of Cyclodextrins. Chem. Rev. (1998); 98, 1875-1917 
32 Roa G. Caracterización electroquímica de una membrana de ciclodextrina y su aplicación en la determinación de complejos de inclusión. Maestra en Química. Universidad Autónoma Metropolitana. (1999); 8-10.

${ }^{33}$ Yang X., Zhao Y., Chen Y., Liao X., Gao Ch., Xiao D., Yi D., Yang B., Qin Q. Host-guest inclusion system of mangiferin with $\beta$-cyclodextrin and its derivatives. Materials Science and Engineering $C$. (2013); 33, 2386-2391.

${ }^{34}$ Huang L., He J., Lu R., Ge X., Guo J. Investigation on a host-guest inclusion system by $\beta$ cyclodextrin derivative and its analytical application. Bioorganic \& Medicinal Chemistry Letters. (2011); 21, 1113-1117.

${ }^{35}$ Elbashir A., Nuha Fathi Ali Dsugi, Tamador Omer Mohamoud Mohmed and Hassan Y. Aboul-Enein Spectrofluorometric analytical applications of cyclodextrins. Luminescence. (2019); 29, 1-7.

${ }^{36}$ Lakowicz J. R. Principles of Fluorescence Spectroscopy. Springer: New York. (2006) 3-7.

${ }^{37}$ MINITAB Statistical Software. Release 13.1. BCIS Lab. St. Cloud State University. Minitab Inc. Programa computacional.

38 Tauler, R.; Smilde, A.; Kowalski, B., Selectivity, local rank, three-way data analysis and ambiguity in multivariate curve resolution. Journal of Chemometrics. (1995); 9(1), 31-58.

${ }^{39}$ D.J. Leggett, Computational Methods for the Determination of Formation Constants, Plenum Press: New York. (1985); 159-217.

40 Islas-Martínez, J. M.; Rodríguez-Barrientos, D.; Galano, A.; Ángeles, E.; Torres, L. A.; Olvera, F.; Ramírez-Silva, M. T.; Rojas-Hernández, A., Deprotonation Mechanism of New Antihypertensive Piperidinylmethylphenols: A Combined Experimental and Theoretical Study. The Journal of Physical Chemistry B. (2009); 113(34), 11765-11774.

41 Rojas A.; González, I. Relationship of two-dimensional predominance-zone diagrams with conditional constants for complexation equilibra. Anal. Chim. Acta. (1986); 187, 279-285.

42 Higuchi T, Connors KA. Phase-solubility techniques. Adv Anal Chem Instrum. (1965); 4, 117-212.

${ }^{43}$ Connors K. The Stability of Cyclodextrin Complexes in Solution. Chem. Rev. (1997); 7, 1325-135.

${ }^{44}$ Acosta J., Sevilla I., Salomón S., Nuevas L., Romero A., Amaro D. Determination of mangiferin solubility in solvents used in the biopharmaceutical industry. Journal of Pharmacy \& Pharmacognosy Research. (2016); 4(2) 49-53.

45 J.D. Van der Merwe, E. Joubert, M. Manley, D. de Beer, C.J. Malherbe, W.C. Gelderblom Mangiferin glucuronidation: important hepatic modulation of antioxidant activity Food Chemistry Toxicology. (2012); 50(3-4), 808-815.

${ }^{46}$ De Souza, J. R. R., Feitosa, J. P. A., Ricardo, N. M. P. S., Trevisan, M. T. S., de Paula, H. C. B., Ulrich, C. M., \& Owen, R. W. (2013). Spray-drying encapsulation of mangiferin using natural polymers. Food Hydrocolloids, (2013); 33(1), 10-18.

47 Miladi K., Sfar S., Fessi H., Elaissari A. Drug carriers in osteoporosis: Preparation, drug encapsulation and applications. International Journal of Pharmaceutics. (2013); 445, 181-195. 
48 Dos Santos C., Buera Ma., Mazzobre Ma. F. Phase solubility studies of terpineol with $\beta$ cyclodextrins and stability of the freeze-dried inclusion complex. Procedia Food Science. (2011); 1 , 355-362.

49 Szaniszlo N., Fenyvesi E., Balla J. Structure-stability Study of Cyclodextrin Complexes with Selected Volatile Hydrocarbon Contaminants of Soils. Journal of Inclusion. Phenomena and Macrocyclic Chemistry. (2005); 53, 241-248.

50 Doile M. M., Fortunato K., Schmücker Í., Schucko S. Physicochemical Properties and Dissolution Studies of Dexamethasone Acetate- $\beta$-Cyclodextrin Inclusion Complexes Produced by Different Methods. AAPS PharmSciTech. (2008); 9(1), 314-321.

51 Crestani J., Azevedo T., Veiga F. Gomes H. Cyclodextrin and ternary complex: techology to improve solubility of poorly solubility of poorly soluble drugs. Brazilian Journal of Pharmaceutical Sciences. (2011); 47(4), 665-681.

52 O. V. Popova, V. V. Sursyakova, G. V. Burmakina, V. A. Levdansky, and A. I. Rubaylo. Determination of Stability Constants of Inclusion Complexes of Betulin Derivatives with $\beta$-Cyclodextrin by Capillary Electrophoresis. Doklady Chemistry. (2015), 461(1), 67-69.

53 M.K. Manolikar, M.R. Sawant. Study of solubility of isoproturon by its complexation with bcyclodextrin. Chemosphere. (2003); 51, 811-816.

${ }^{54}$ Karathanos V., Mourtzinos I., $\quad$ Study of the solubility, antioxidant activity and structure of inclusion complex of vanillin with $\beta$-cyclodextrin. Food Chemistry. (2007); 101, 652-658.

${ }^{55}$ Rojas-Hernández, A.; Ramírez, M.T.; González, I.; Ibanez, J. G. Predominance-Zone Diagrams in Solution Chemistry. J. Chem. Educ. (1995); 72, 1099-1105. 
APÉNDICES 


\section{Apéndices}

\section{A. Modelo de tres equilibrios para determinar relaciones molares}

La construcción del método de tres equilibrios se propuso porque en los equilibrios del tipo huésped-anfitrión pueden presentarse reacciones químicas poco cuantitativas. Debido a esto no es posible utilizar el método clásico de relaciones molares para determinar la estequiometría de una reacción.

Se considera entonces un sistema con las especies químicas $G(C D), G_{2}(C D)$ y $G(C D)_{2}$, donde $G$ representa al huésped y $C D$ al anfitrión. Cada especie aparece por su equilibrio de formación correspondiente, como se establece en las ecuaciones (D.1) a la (D.3), con sus constantes de inclusión asociadas.

$$
\begin{aligned}
& G+C D \rightarrow G(C D) \quad \beta_{11}=K_{1: 1}=\frac{[G(C D)]}{[G][C D]} \\
& G+2 C D \rightarrow G(C D)_{2} \quad \beta_{12}=K_{1: 2}=\frac{\left[G(C D)_{2}\right]}{[G][C D]^{2}} \\
& 2 G+C D \rightarrow G_{2}(C D) \quad \beta_{21}=K_{2: 1}=\frac{\left[G_{2}(C D)\right]}{[G]^{2}[C D]}
\end{aligned}
$$

Por otra parte, se puede escribir la ecuación de balance del componente $\mathrm{G}$, tal que:

$$
[G]_{\text {total }}=[G]+[G(C D)]+\left[G(C D)_{2}\right]+2\left[G_{2}(C D)\right]
$$

Despejando los complejos de inclusión de las Ecuaciones D.1 a D.3 y sustituyendo en la Ecuación D.4, se llega a la Ecuación D.5:

$$
[G]_{\text {total }}=[G]+[G][C D] \beta_{1: 1}+[G][C D]^{2} \beta_{1: 2}+2[G]^{2}[C D] \beta_{2: 1}
$$

Donde el sistema debe cumplir con la restricción de que $C_{(C D)_{\text {otoll }}} \geq C_{G_{\text {total }}}$.

Por lo tanto, es posible calcular las fracciones de cantidad de las especies formadas en términos de Gtotal, dadas por. 


$$
\begin{gathered}
f_{G}=f_{10}=\frac{[G]}{[G]_{\text {total }}}=\left(\frac{1}{1+\beta_{11}[\mathrm{CD}]+\beta_{12}[\mathrm{CD}]^{2}+2 \beta_{21}[G][\mathrm{CD}]}\right) \\
f_{G(C D)}=f_{1: 1}=\frac{[\mathrm{G}(\mathrm{CD})]}{[G]_{\text {total }}}=f_{10} \beta_{11}[\mathrm{CD}] \\
f_{G(C D)_{2}}=f_{1: 2}=\frac{\left[\mathrm{G}(\mathrm{CD})_{2}\right]}{[G]_{\text {total }}}=f_{10} \beta_{12}[\mathrm{CD}]^{2} \\
f_{G_{2}(C D)}=f_{21}=\frac{2\left[G_{2}(C D)\right]}{[G]_{\text {total }}}=\frac{2 \beta_{21}[G]^{2}[C D]}{[G]_{\text {total }}}=2 f_{10} \beta_{21}[G][C D]=2 f_{10}^{2} \beta_{21}[G]_{\text {total }}[C D]
\end{gathered}
$$

Donde $[G]$ total es la suma de las concentraciones de las especies del componente G. Finalmente, con esta información se puede construir el diagrama de distribución de especies en términos de la cantidad de $\mathrm{pCD}$, resolviendo antes la ecuación cuadrática que aparece en la fracción $f_{G_{2}(C D)}$. 


\section{B. Análisis de Componentes Principales}

El método de Análisis de Componentes Principales (PCA por sus siglas en inglés) es una técnica estadística utilizada para reducir la dimensionalidad de un conjunto de datos. A partir de un procedimiento matemático que transforma un conjunto de variables correlacionadas de respuesta en un conjunto menor de variables no correlacionadas llamadas componentes principales.

El análisis de componentes principales es útil si se desea agrupar las unidades experimentales en subgrupos de tipos semejantes. Se puede usar para ayudar a identificar agrupamientos de las unidades experimentales en subgrupos o para verificar los resultados de los programas de agrupación.

La matriz contiene la información de los datos experimentales, en este caso los espectros de absorbancia o bien los espectros de excitación y emisión a diferentes valores de $\mathrm{pH}$.

Se utilizó el Software. Minitab 16. La matriz está compuesta de muestras (pH) y las variables (longitudes de onda), cabe mencionar que para cada valor de $\mathrm{pH}$ se tienen $\mathrm{n}$ longitudes de onda y un valor de la respuesta medida espectroscópica.

La matriz $\mathrm{X}$, con un valor de $\lambda$, se descompone en tres matrices:

$$
X=U S V^{T}
$$

Donde las columnas de $\mathrm{V}^{\top}$ son los eigenvalores; $\mathrm{S}$ es una matriz diagonal cuyos valores son las raíces cuadradas de los eigenvalores; y las columnas U se llaman "scores" y están relacionados con las muestras.

Las columnas de $\mathrm{V}^{\top}$ se llaman "loadings" y están relacionadas con las variables. Esta matriz es la reconstrucción de la matriz original con la mayor información relevante al $100 \%$. 


\section{Método computacional SQUAD}

El programa SQUAD (Stability QUotients from Absorbance Data), escrito en un lenguaje FORTRAN. Creado para conocer las constantes de formación de diferentes especies, las cuales son de gran importancia debido a la valiosa información que se obtiene de éstas, pues se pueden encontrar con mayor facilidad las condiciones de trabajo óptimas para las experimentaciones.

La determinación de constantes utiliza diferentes métodos analíticos, de los cuales el más empleado es la espectrofotometría UV-visible ${ }^{39}$. Y actualmente también se ha visto que puede utilizarse los espectros de emisión y excitación de Fluorescencia.

SQUAD además diseñado para calcular los mejores valores para las constantes de estabilidad para un modelo propuesto, esto lo realiza por minimización de la suma de residuales al cuadrado de los valores de las absorbancias.

Es capaz de calcular simultáneamente o individualmente constantes de estabilidad global, para cualquier especie formada en sistemas que contienen hasta dos metales y dos ligantes, a partir de datos de absorbancia experimentales a diferentes longitudes de onda. El modelo químico puede ser dependiente o independiente de $\mathrm{pH}$.

Por esta razón este programa es una buena opción para estudios de equilibrios ácido-base para ligandos que pueden ser ácidos o bases débiles, $M_{m} L_{n}$ complejos mixtos de metales con ligandos, complejos protonados o hidroxocomplejos.

Para un número $i$ de soluciones medidas y un número $k$ de longitudes de onda, la absorbancia $A_{i k}$ se define por la ecuación siguiente:

$$
A_{i j}=l \sum \varepsilon_{k j} C_{j}
$$

donde $I_{k j}$ es la absortividad molar de cada una de las especies $\mathrm{Mp}_{\mathrm{p}} \mathrm{q}_{\mathrm{r}}$ a cada longitud de onda $\mathrm{K}, \varepsilon$ es la longitud del paso óptico y $\mathrm{C}_{\mathrm{j}}$ es la concentración de cada especie, SQUAD computa los valores de las constantes de formación global 
minimizando la suma de cuadrados que existe entre los valores de absorbancia observados experimentalmente y los valores de absorbancia calculados por el programa.

A continuación se da una lista general que datos hay que alimentar y como funciona SQUAD:

$\checkmark$ Se alimentan datos: Espectrofotométricos y modelos químicos (estequiométricos).

$\checkmark$ Puede utilizarse valores de $\mathrm{pH}$, para cada espectro 0 bien puede ser independiente variando la concentración del metal.

$\checkmark$ Método empleado es por minimización de la suma de cuadrados residuales de las absorbancias.

Se obtienen los siguientes parámetros estadísticos que emplea SQUAD para determinar si el modelo químico propuesto explica la información experimental alimentada son los siguientes: Parámetros de correlación (matriz), desviación estándar sobre los datos de absorbancia (Respuesta) y sobre las constantes, desviación estándar por espectro, desviación estándar por coeficiente de absortividad molar (o bien el factor de respuesta dependiendo la técnica de estudio) Las constantes refinadas deben de cumplir el criterio de convergencia, la cual se da si la diferencia en la minimización de un ciclo iterativo a otro difiere como máximo en 0.001 . Cabe mencionar que si no se cumple lo anterior no es indicativo que se tiene el mejor refinamiento.

Este modelo se puede utilizar con diferentes tipos de respuestas experimentales, como fue en el caso de este trabajo, usando la intensidad de Fluorescencia, ya que cumple con la ley de Biot y una ley de aditividad. Para poder alimentar los espectros de fluorescencia al programa SQUAD es necesario escalando la respuesta de Intensidad de Fluorescencia a valores que se encuentren entre 0 y 3 , lo que normalmente se logra dividiendo la respuesta por un factor de 100 o 1000. Para interpretar los resultados debe tomarse en cuenta este escalamiento. 


\section{Dependencia de las constantes condicionales de acidez con [BCD]}

Si se expresa la concentración de la especie generalizada $\mathrm{H}_{4} \mathrm{MGF}^{\prime}$ :

$$
\left[H_{4} M G F^{\prime}\right]=\left[H_{4} M G F\right]+\left[H_{4} M G F(\beta C D)\right]+\left[H_{4} M G F(\beta C D)_{2}\right]+\ldots(\mathrm{D} .1)
$$

En las ecuaciones D.2 y D.3 se presentan los equilibrios de formación global de las especies de $\mathrm{H}_{4} \mathrm{MGF}$ con $\beta C D$.

$$
\begin{aligned}
& H_{4} M G F+\beta C D=H_{4} M G F(\beta C D) \quad \text { con } \quad K_{H_{4} M G F(\beta C D)}^{\beta C D}=\frac{\left[H_{4} M G F(\beta C D)\right]}{\underline{\left[H_{4} M G F\right][\beta C D]}} \\
& H_{4} M G F+2 \beta C D=H_{4} M G F(\beta C D)_{2} \quad \text { con } K_{H_{4} M G F(\beta C D)_{2}}^{2 \beta C D}=\frac{\left[H_{4} M G F(\beta C D)_{2}\right]}{\underline{\left[H_{4} M G F\right][\beta C D]^{2}}}
\end{aligned}
$$

Si se despeja de las ecuaciones D.2 y D.3 las concentraciones de las especies $\mathrm{H}_{4} \mathrm{MGF}(\beta C D)$ y $\mathrm{H}_{4} M G F(\beta C D)_{2}$ y se sustituyen en la ecuación D.1, se obtiene la ecuación D.4.

$$
\left[H_{4} M G F^{\prime}\right]=\left[H_{4} M G F\right]\left(1+K_{H_{4} M G F(\beta C D)}^{\beta C D}[\beta C D]+K_{H_{4} M G F(\beta C D)_{2}}^{2 \beta C D}[\beta C D]^{2}+\ldots\right)
$$

Si se define el coeficiente de complejación de $\mathrm{H}_{4} \mathrm{MGF}$ dependiente de $\beta C D$, de acuerdo a la ecuación D.5:

$$
\alpha_{H_{4} M G F(\beta C D)}=1+K_{1+K_{H_{4}}^{\beta C D C F(\beta C D)}}^{\beta C D}[\beta C D]+K_{H_{4} M G F(\beta C D)_{2}}^{2 \beta C D}[\beta C D]^{2}+\ldots
$$

Entonces la ecuación D.4 se puede escribir como se presenta en la ecuación D.6.

$$
\left[H_{4} M G F^{\prime}\right]=\left[H_{4} M G F\right] \alpha_{H_{4} M G F(\beta C D)}
$$

Si se sigue el mismo procedimiento algebraico mostrado en las ecuaciones D.1 a D.6 se puede representar cada una de las especies generalizadas como se muestra en las ecuaciones D.7 a D.10. 


$$
\begin{aligned}
{\left[H_{3} M G F^{\prime}\right] } & =\left[H_{3} M G F^{-}\right] \alpha_{H_{3} M G F(\beta C D)} \\
{\left[H_{2} M G F^{\prime}\right] } & =\left[H_{2} M G F^{2-}\right] \alpha_{H_{2} M G F(\beta C D)} \\
{\left[H M G F^{\prime}\right] } & =\left[H M G F^{3-}\right] \alpha_{H M G F(\beta C D)} \\
{\left[M G F^{\prime}\right] } & =\left[M G F^{4-}\right] \alpha_{M G F(\beta C D)}
\end{aligned}
$$

Si se utilizan las ecuaciones de la D.7 a la D.10 y se sustituyen en las constantes condicionales de acidez que se definieron en las ecuaciones 3.6 a la 3.9, se obtiene las ecuaciones D.11 a la D.14.

$$
\begin{gathered}
K_{a 1}^{\prime} \equiv \frac{\left[H_{3} M G F^{\prime}\right]\left[H^{+}\right]}{\left[H_{4} M G F^{\prime}\right]}=\frac{\left[H_{3} M G F^{-}\right] \alpha_{H_{3} M G F(\beta C D)}\left[H^{+}\right]}{\left[H_{4} M G F\right] \alpha_{H_{4} M G F(\beta C D)}}=K_{a 1} \frac{\alpha_{H_{3} M G F(\beta C D)}}{\alpha_{H_{4} M G F(\beta C D)}} \\
K_{a 2}^{\prime} \equiv \frac{\left[H_{2} M G F^{\prime}\right]\left[H^{+}\right]}{\left[H_{3} M G F^{\prime}\right]}=\frac{\left[H_{2} M G F^{2-}\right] \alpha_{H_{2} M G F(\beta C D)}\left[H^{+}\right]}{\left[H_{3} M G F^{-}\right] \alpha_{H_{3} M G F(\beta C D)}}=K_{a 2} \frac{\alpha_{H_{2} M G F(\beta C D)}}{\alpha_{H_{3} M G F(\beta C D)}} \\
K_{a 3}^{\prime} \equiv \frac{\left[H M G F^{\prime}\right]\left[H^{+}\right]}{\left[H_{3} M G F^{\prime}\right]}=\frac{\left[H M G F^{3-}\right] \alpha_{H M G F(\beta C D)}\left[H^{+}\right]}{\left[H_{2} M G F^{2-}\right] \alpha_{H_{2} M G F(\beta C D)}}=K_{a 3} \frac{\alpha_{H M G F(\beta C D)}}{\alpha_{H_{2} M G F(\beta C D)}} \\
K_{a 4}^{\prime} \equiv \frac{\left[M G F^{\prime}\right]\left[H^{+}\right]}{\left[H M G F^{\prime}\right]}=\frac{\left[M G F^{4-}\right] \alpha_{M G F(\beta C D)}\left[H^{+}\right]}{\left[H M G F^{3-}\right] \alpha_{H M G F(\beta C D)}}=K_{a 3} \frac{\alpha_{M G F(\beta C D)}}{\alpha_{H M G F(\beta C D)}}
\end{gathered}
$$

En las ecuaciones D.11 a D.14 se muestra la dependencia de las constantes de acidez condicionales con la concentración de $\beta C D$. Esto demuestra que si se mantiene constante la concentración de $\beta C D$ en el sistema, las constantes de acidez condicionales también serán constantes pero diferentes a las constantes de acidez, si es que se da la formación de los complejos de inclusión. 


\section{E. Constantes de equilibrio globales ( $\beta$ )}

En química de soluciones, es frecuente considerar los equilibrios globales de formación como un buen conjunto de equilibrios independientes para simular e interpretar el comportamiento químico de este tipo de sistemas polidonadores. En general, el conjunto de equilibrios globales de formación, puede representarse a través de la ecuación G.1.

$$
M+j L=M L_{j} \quad \text { con } \quad \beta_{j}=K_{M L_{j}}^{M, j L}=K_{M L_{j}}^{j L}=\frac{\left[M L_{j}\right]}{[M][L]^{j}}=\frac{1}{K_{M, j L}^{M L_{j}}}=\frac{1}{K_{j L}^{M L_{j}}}
$$

donde $j \epsilon\{1,2, \ldots, n\}$

Los equilibrios sucesivos de formación son equivalentes a los sucesivos de disociación. Estos equilibrios pueden representarse a través de la ecuación general G.2.

$$
M L_{(j-1)}+L=M L_{j} \quad \text { con } \quad K_{f j}=K_{M L_{j}}^{L}=\frac{\left[M L_{j}\right]}{\left[M L_{(j-1)}\right][L]}=\frac{1}{K_{L}^{M L_{j}}}
$$

donde $j \epsilon\{1,2, \ldots, n\}$

Por ejemplo, los equilibrios globales de formación de mangiferina se definen en las ecuaciones G.3 a la G.6

$$
\begin{array}{cc}
M G F^{4-}+H^{+}=H M G F^{3-} & \log \beta_{1}=12.10 \\
M G F^{4-}+2 H^{+}=H_{2} M G F^{2-} & \log \beta_{2}=21.54 \\
M G F^{4-}+3 H^{+}=H_{3} M G F^{-} & \log \beta_{3}=29.51 \\
M G F^{4-}+4 H^{+}=H_{4} M G F & \log \beta_{4}=36.03
\end{array}
$$

Para conocer los equilibrios sucesivos de disociación y determinar el valor de las constantes de acidez, como es el caso del equilibrio sucesivo de la ecuación G.7, 
se aplica la ley de Hess para los equilibrios de formación G.5 y G.6, recordando la definición $p K_{a i}=-\log K_{a i}$

$$
\begin{aligned}
& H_{4} M G F=M G F^{4-}+4 H^{+} \quad \log K_{I}=-\log \beta_{4}=-36.03 \\
& M G F^{4-}+3 H^{+}=H_{3} M G F^{-} \quad \log K_{I I}=\log \beta_{3}=29.51 \\
& H_{4} M G F=H_{3} M G F^{-}+H^{+} \quad \log \mathrm{K}_{a 1}=-6.52 \Rightarrow p K_{a 1}=6.52
\end{aligned}
$$

Este procedimiento se puede aplicar para los equilibrios sucesivos representados por las ecuaciones G.7 a la G.10.

$$
\begin{gathered}
H_{3} M G F^{-}=M G F^{4-}+3 H^{+} \quad \log \mathrm{K}_{I I I}=-\log \beta_{3}=-29.51 \\
M G F^{4-}+2 H^{+}=H_{2} M G F^{2-} \quad \log K_{I V}=\log \beta_{2}=21.54 \\
H_{3} M G F^{-}=H_{2} M G F^{2-}+H^{+} \quad p K_{a 2}=7.97 \\
H_{2} M G F^{2-}=M G F^{4-}+2 H^{+} \quad \log K_{V}=-\log \beta_{2}=-21.54 \\
M G F^{4-}+H^{+}=H M G F^{3-} \quad \log K_{V I}=\log \beta_{1}=12.10 \\
\quad H_{2} M G F^{2-}=H M G F^{3-}+H^{+} \quad p K_{a 3}=9.44 \\
H M G F^{3-}=M G F^{4-}+H^{+} \quad \log K_{V I I}=-\log \beta_{1}=-12.10 \\
H M G F^{3-}=M G F^{4-}+H^{+} \quad p K_{a 4}=12.10
\end{gathered}
$$




\section{F. Diagramas de distribución de especies para sistemas ácido-base}

Las representaciones gráficas de los sistemas químicos han encontrado una amplia aplicación que permite resolver problemas específicos y tener un panorama, cualitativo y cuantitativo. A partir del estudio de los sistemas polípticos, cuyo equilibrio de formación global está representado en la ecuación. D.1, en solución acuosa que contiene dos componentes $\mathrm{M}$ y L.

$$
L^{a-}+j\left[H^{+}\right]=L H_{j}^{j-a} \quad \text { con } \beta_{j}=\frac{\left[L H_{j}^{j-a}\right]}{\left[L^{a-}\right]\left[H^{+}\right]^{j}}
$$

En el sistema de estudio de este trabajo, las especies químicas $L$ y $M$, están referidas respecto al protón $\left(\mathrm{H}^{+}\right)$y mangiferina $\left(\mathrm{MGF}^{4-}\right)$, respectivamente.

La construcción del diagrama de distribución de especies, se define típicamente con las fracciones molares del componente $\mathrm{M}$ que depende de concentración de $\mathrm{L}^{55}$, ecuación D.2.

$$
f_{j}=\frac{\left[L H_{j}^{j-a}\right]}{[L]_{T}}=\frac{\beta_{j}\left[H^{+}\right]^{j}}{1+\sum_{j=1}^{n} \beta_{j}\left[H^{+}\right]^{j}}
$$

donde $j \epsilon\{0,1, \ldots, n\}$

El diagrama de distribución de especies es la gráfica de fracciones molares en función del pH.

Para construir el diagrama de distribución de especies se utiliza una hoja de cálculo de Excel, que calcula las fracciones molares con el conjunto de ecuaciones D.2 


\section{G. Diagrama de solubilidad-fases}

Uno de los enfoques analíticos más útiles y ampliamente aplicados para determinar la solubilidad de alguna sustancia orgánica en la formación de complejos con ciclodextrina es el método de solubilidad-fases descrito por Higuchi y Connors ${ }^{42}$. El diagrama de solubilidad-fases se clasifica en dos categorías, de tipo A y B. Las curvas de tipo-A son indicativas para la formación de complejos de inclusión solubles del huésped $(G)$ que satura la solución, mientras que el comportamiento de tipo-B es la formación de complejos de inclusión de poca solubilidad o presentes en la fase sólida.

Estos sistemas de solubilidad-fases no sólo permiten una evaluación cualitativa de los complejos formados, sino que también pueden usarse para determinar constantes de equilibrio $(K)$ para la formación de $\left[G_{m} C D_{n}\right]$, que puede ser representado por:

$$
K=\frac{\left[G_{m} C D_{n}\right]}{[G]^{m}[C D]^{n}}
$$

Donde, por el balance de materia:

$$
\begin{gathered}
{[G]=G_{0}} \\
{[G]_{t}=G_{0}+m\left[G_{m} C D_{n}\right]} \\
{[C D]_{t}=C D+n\left[G_{m} C D_{n}\right]}
\end{gathered}
$$

Por lo tanto, los valores de $\left[G_{m} C D_{n}\right],[G]$ y $[C D]$, se pueden obtener por:

$$
\begin{gathered}
{[G]=G_{0}} \\
{\left[G_{m} C D_{n}\right]=\frac{[G]_{t}-G_{0}}{m}} \\
C D=[C D]_{t}-n\left[G_{m} C D_{n}\right]
\end{gathered}
$$


Donde $G_{0}$ es la solubilidad en el equilibrio de $G$ (es la molécula Huésped), [G] tes la concentración total de $\mathrm{G}$ (en la formación del complejo y la libre) y [CD]t es la concentración total de CD. Para el sistema solubilidad de fases de primer orden para este trabajo, con respecto a la ciclodextrina si supone $(n=1)$, se deriva la siguiente ecuación de la sustitución de (E.5), (E.6) y (E.7) en (E.1):

$$
G_{t}=\frac{m K G_{0}[C D]_{t}}{1+K G_{0}^{m}}+G_{0}
$$

Con esto se puede realizar un gráfico de $[G]_{t}$ en función de $[C D]_{t}$ para la formación de $G_{m} C D$, que debe resultar una línea recta con la intersección en el eje $y$ que representa $G_{0}$ y con pendiente:

$$
\text { slope }=\frac{m K G_{0}}{1+K G_{0}^{m}}
$$

Por lo tanto, si $\boldsymbol{m}$ se conoce, $\mathrm{K}$ puede ser calculada. En este trabajo se determinó una estequiometría 1:1, para la formación del complejo, entonces $m=1$, por lo que puede ser aplicada la siguiente ecuación:

$$
K_{1: 1}=\frac{\text { slope }}{G_{0}(1-\text { slope })}
$$

Slope es la pendiente en el diagrama de fases cuando presenta una tendencia lineal y se representa por $\alpha$. 


\section{H. Polarimetría}

La Polarimetría es una técnica usada como un método instrumental de análisis, que se basa en la medición de la rotación óptica producida sobre un haz de luz linealmente polarizada al pasar por una sustancia ópticamente activa. La actividad óptica rotatoria de una sustancia tiene su origen en la asimetría estructural de las moléculas.

Un compuesto es considerado ópticamente activo si la luz linealmente polarizada sufre una rotación cuando pasa a través de una muestra de dicho compuesto. La actividad óptica viene determinada por la estructura molecular y la concentración de moléculas quirales.

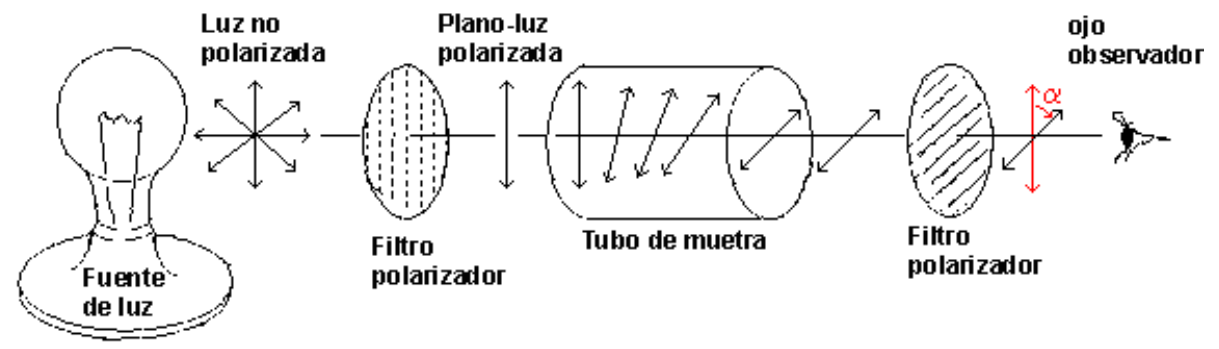

La Polarimetría está relacionada con la rotación $(\alpha)$ del plano de la luz linealmente polarizada y geometría del sistema ópticamente activo, se puede conocer la ley de Biot:

$$
\alpha=[\alpha]_{\lambda}^{T} l c
$$

Donde se conoce a $\alpha$, como el ángulo de rotación medido en grados sexagesimales; I, es la longitud de la celda que se mide en $\mathrm{mm}$ para sólidos y en dm para líquidos; c, es la concentración de la muestra analizada en $\mathrm{g} \mathrm{dL}^{-1}$, finalmente $[\alpha]_{\lambda}^{T}$, es la rotación especifica que depende de la temperatura de trabajo $(\mathrm{T})$ y la longitud de onda de medición, que por lo general utiliza linea $\mathrm{D}$ del sodio cuya longitud de onda es de $589 \mathrm{~nm}$.

Las sustancias pueden ser dextrógiro si giran el ángulo de rotación hacia valores positivos (+) y las que lo giran a valores negativos (-) son conocidas como levógiras. 


\section{Alal \\ UNIVERSIDAD AUTÓNOMA METROPOLITANA \\ Unidad Iztapalapa}

Fecha : $26 / 07 / 2017$

Páqina : $\quad 1 / 1$

CONSTANCIA DE PRESENTACION DE EXAMEN DE GRADO

La Universidad Autónoma Metropolitana extiende la presente CONSTANCIA DE PRESENTACION DE EXAMEN DE GRADO de MAESTRA EN CIENCIAS (OUIMICA) de la alumna LUCERO HERNANDEZ GARCIA, matrícula 2153804812, quien cumplió con los 132 créditos correspondientes a las unidades de enseñanza aprendizaje del plan de estudio. Con fecha veintiocho de agosto del 2017 presentó la DEFENSA de su EXAMEN DE GRADO cuya denominación es:

Estudio de la interacción de

mangiferina con

$\beta$-ciclodextrina al formar

complejos de inclusión en

medio acuoso.

Cabe mencionar que la aprobación del Examen de Grado tiene un valor de 60 créditos y el programa consta de 183 créditos.

El jurado del examen ha tenido a bien otorgarle la calificación de: Aprubada

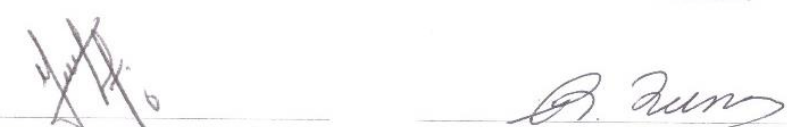

DRA. MARIA TERESA RAMIREZ SILVA

DR. RAFAEL ARTURO ZUBILLAGA LUNA

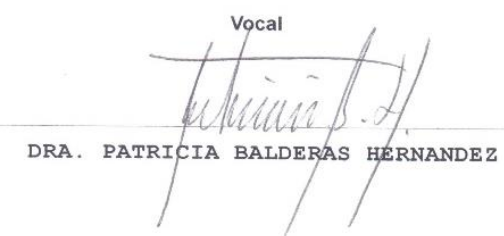

Av. San Rafael Atlixco No. 186. Col. Vicentina, Del. Iztapalapa, C.P. 09340, México, D.F. Tels: 58044880 y 58044883 csera@xanum.uam.mx y cses@xanum.uam.mx http://cse.izt.uam.mx/cse/ 